\title{
EFFECTS OF OVARIECTOMY, SEASONAL CHANGES, AND ANATOMICAL POSITION ON THE COMPACT BONE REMODELING AS SEEN IN THE ADULT OVINE MODEL
}

\author{
A Thesis \\ Presented to \\ the Faculty of California Polytechnic State University, \\ San Luis Obispo
}

\author{
In Partial Fulfillment \\ of the Requirements for the Degree \\ Master of Science in Biomedical Engineering
}

By

Erica Wong

June 2012 
(C) 2012

Erica Wong

ALL RIGHTS RESERVED 
TITLE:

EFFECTS OF OVARIECTOMY, SEASONAL CHANGES, AND ANATOMICAL POSITION ON THE COMPACT BONE REMODELING AS SEEN IN THE ADULT OVINE MODEL
AUTHOR:
Erica Wong
DATE SUBMITTED: June 2012
COMMITTEE CHAIR: Dr. Scott Hazelwood
COMMITTEE MEMBER: Dr. Lanny Griffin
COMMITTEE MEMBER: Dr. Clifford Les 


\title{
ABSTRACT \\ EFFECTS OF OVARIECTOMY, SEASONAL CHANGES, AND ANATOMICAL POSITION ON THE COMPACT BONE REMODELING AS SEEN IN THE ADULT OVINE MODEL
}

\author{
Erica Wong
}

The purpose of this study is to characterize the compact bone remodeling of the ovariectomized ewe and its effectiveness as an animal model for studies of postmenopausal osteoporosis. The use of animal models is a beneficial way to evaluate the effectiveness of medical devices and therapeutic agents for treating diseases. The ovine model is an attractive option because of its large size and similar bone remodeling to humans. However, this species, like most animals, does not undergo a natural menopause, making an ovariectomy necessary for studies of estrogen depletion. The OVX (ovariectomized) sheep has been widely accepted as a model for loss of BMD. However, even with its advantages seasonality has been shown to have an effect on the bone remodeling in the ovine model. Thus, this study focused on characterizing the seasonal and anatomical variation in the compact bone remodeling in the ovine model. In doing so, 28 skeletally mature Columbia-Rambouillet cross ewes underwent an ovariectomy. The animals were divided into groups of 7 based on each season: autumn, winter, spring, and summer. During its specified season each group of 7 underwent ovariectomy and then was sacrificed 12 months post-surgery. The radii and ulnae were harvested and then divided into 6 anatomical locations: craniolateral, cranial, craniomedial, craniolateral, caudal, and caudomedial. This allowed for analysis through the fabrication of microradiographs. Histomorphometric analysis involved measurements to quantify the bone volume to tissue volume ratio, the percent of tissue and material 
remodeling, the mean secondary osteonal radius, and number of tissue and material cement line interfaces. Densitometry analysis was then performed to determine the density of each sector relative to an aluminum step wedge, serving as the key. Using a 2way repeated measures ANOVA statistical analysis was performed to evaluate for seasonal and anatomical differences as well as a possible interaction between season and anatomical region. The OVX data showed significant seasonal and anatomical differences as well as seasonality within anatomical section in the remodeling parameters. When this data was combined with earlier data from a control, sham-cohort from the same experiment a 3-way ANOVA was also performed to evaluate the effects of season, anatomical sector, and treatment on the remodeling parameters. Again, there was significant seasonal, anatomical, and treatment differences, as well as interactions of all three. The results of this study showed that varying levels of remodeling occurs in the adult OVX ovine model and differences between the control and OVX model can be attributed to seasonal and anatomical variations. Thus, it will be an important consideration when developing new test protocols for research incorporating the sheep model for studying osteoporosis.

Keywords: Postmenopausal, Osteoporosis, OVX, Ovariectomy, Ewe, Bone Remodeling 


\section{ACKNOWLEDGEMENTS}

I would like to thank Dr. Hazelwood for the opportunity to be a part of his laboratory and work on this project. Through your classes I formed a solid foundation that allowed me to accomplish the goals of this project. I would also like to thank Dr. Clifford Les for providing me the opportunity to work on this project. Your guidance and patience have been truly appreciated, and without it this project would not have been possible. I would also like to thank Joe Calcagno for his assistance in the preliminary research. Additionally, I would like to thank him for training me and getting me accustomed to the proper methods for completing this project. Finally, I would like to thank all of my friends and family for their undying support and encouragement.

This project was supported by NIH-NIAMS AR 47434. 


\section{TABLE OF CONTENTS}

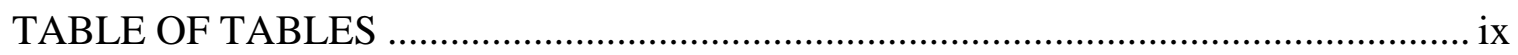

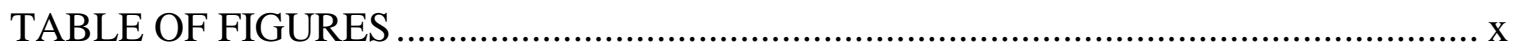

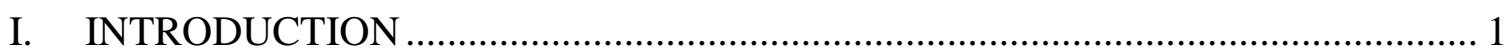

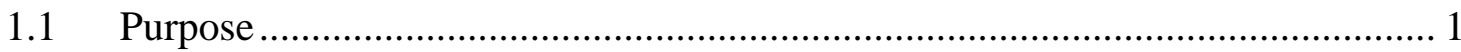

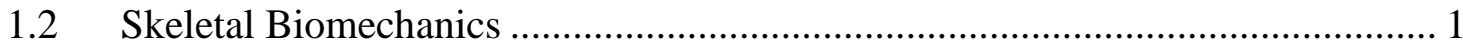

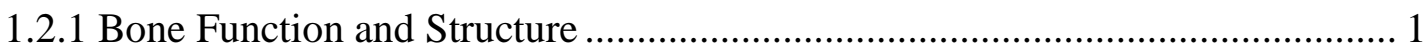

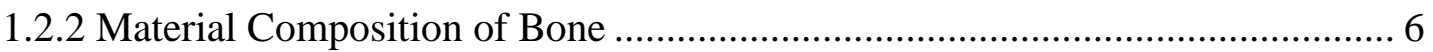

1.2.3 Quantitative Analysis of Skeletal Biomechanics.............................................. 9

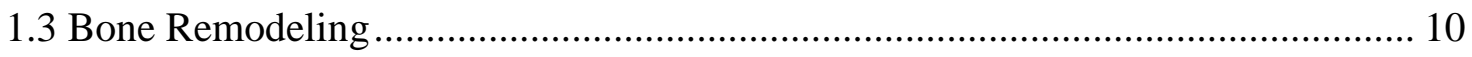

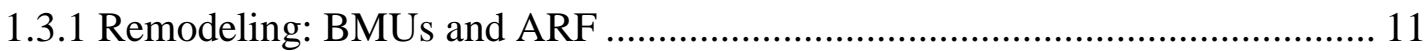

1.3.2 Signaling and Initiation of Bone Remodeling ................................................. 14

1.3.3 Quantitative Analysis of Bone Remodeling ..................................................... 15

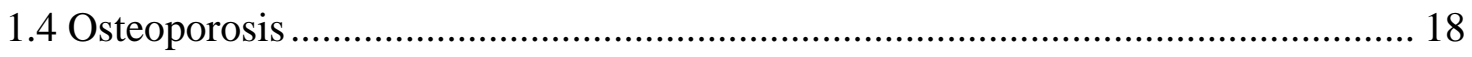

1.4.2 Postmenopausal Osteoporosis ................................................................. 19

1.4.3 Treatment Options for Osteoporosis........................................................... 22

1.5 Necessity for Animal Models............................................................................ 24

1.5.1 Proposed Animal Model......................................................................... 26

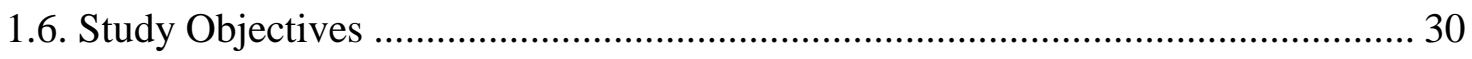

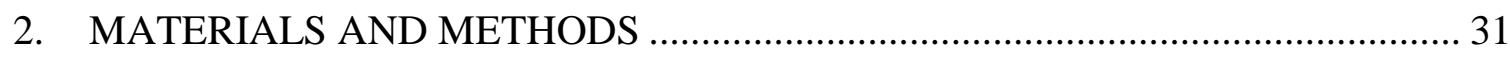

2.1 Animal Maintenance and Preparation ................................................................... 31

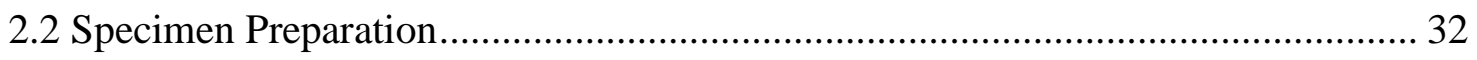

2.4 Microradiograph Analysis ............................................................................ 34

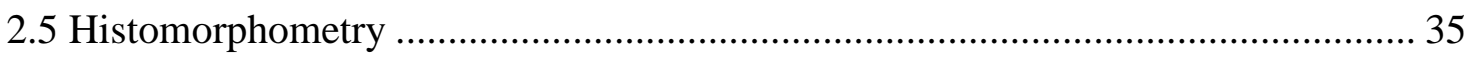

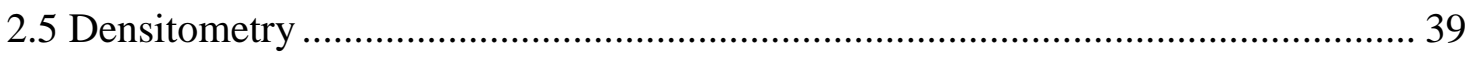

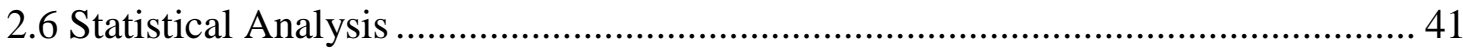

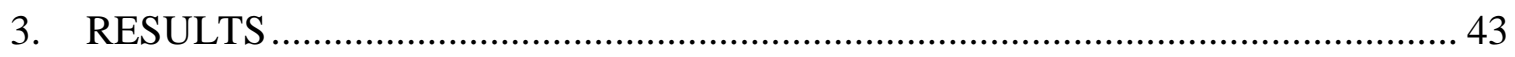

3.1 Histomorphometry ...................................................................................... 43

3.1.2 Two Way Repeated Measures ANOVA............................................................ 46

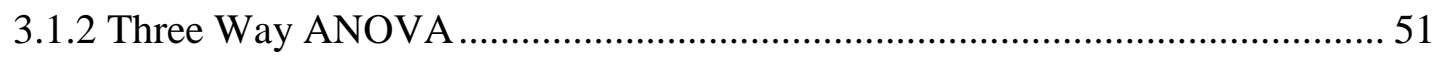




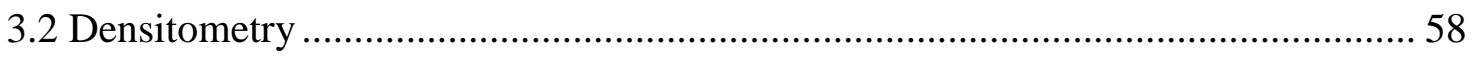

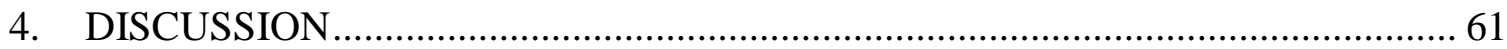

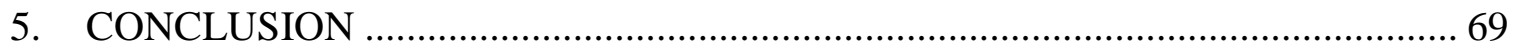

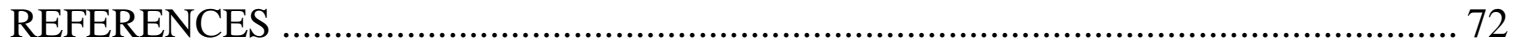

Appendix A: 12 Month OVX Histomorphometry Data ............................................. 75

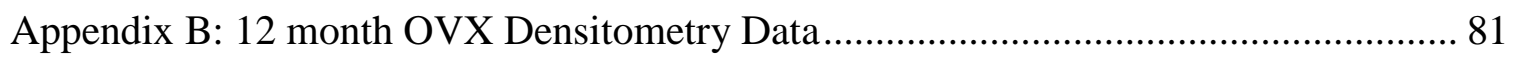




\section{TABLE OF TABLES}

Table I Bone volume to tissue volume for adult 12 month OVX ovine compact bone.... 43

Table II Percent remodeled tissue for adult 12 month OVX ovine compact bone ........... 43

Table III Percent remodeled material for adult 12 month OVX ovine compact bone...... 44

Table IV Mean secondary osteon radius for adult 12 month OVX ovine compact bone. 44

Table V Cement line interfaces (tissue) for adult 12 month OVX ovine compact bone.. 45

Table VI Cement line interfaces (material) for adult 12 month OVX ovine compact

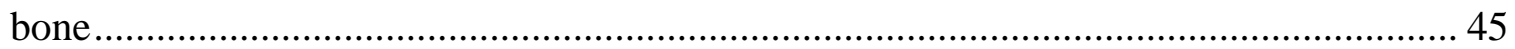

Table VII P-values for 2-way repeated measures ANOVA of remodeling parameters ... 46

Table VIII P-values for 3-way ANOVA for comparison of OVX and control

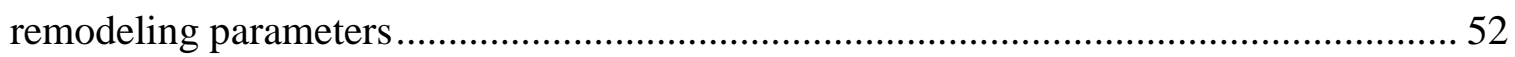

Table IX Densitometry values (ETA) for OVX sheep ............................................ 59 


\section{TABLE OF FIGURES}

Figure 1 Bone structure for cortical and trabecular bone [7] ..........................................

Figure 2 The relationships between ultimate compress stress, porosity, and apparent density for fresh human bone (triangles), embalmed human bone (circles),

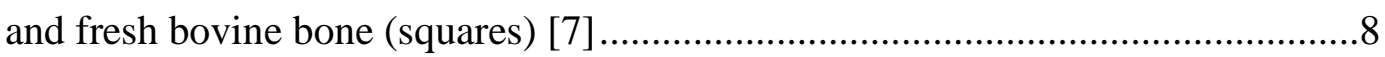

Figure 3 Merz grid with point markers and sinusoidal lines ...........................................10

Figure 4 The Remodeling Cycle on a Trabeculum [11] ................................................11

Figure 5 Tunneling of osteoclasts to form a new osteon [13].........................................13

Figure 6 Upper: Schematic diagram of BMUs in a cross section of cortical bone.

Black, irregularly shaped BMUs represent resorption spaces (R). BMUs with large canals are refilling; they have either a single label (F, S) or a double label (F, D). Completed osteons have small canals @; some of these have a single label (C, S). Lower: A single BMU in the last stages of refilling is depicted. It has a double label; the distance between the labels is measured as shown $\left(\mathrm{D}_{\mathrm{L}}\right)[7]$

Figure 7 The structure of L3 vertebra in a 31 year old woman (top) and 70 year old woman (bottom) is shown using scanning electron micrographs. Note that many of the plate-like structures have become converted to thin rods [4] ............18

Figure 8 Cell signaling pathway occurring during estrogen deficiency

Figure 9 Regulation of IL-1, IL-6, and TNF production. Each arrow indicates a stimulatory effect of a cytokine on its own synthesis, or the synthesis of another cytokine. Demonstrated suppression of the stimulated synthesis of IL-6 and TNF by estrogen ("E") is indicated by filled octagons, and the potential suppression of IL- 6 induced IL- 6 by estrogen is indicated by an open octagon [22]

Figure 10 Lumbar spinal bone mineral density (BMD) increased similarly in estrogen and etidronate treated patients and by a greater amount using both. EHDP=etidronate; HRT=hormone replacement therapy [24] ...

Figure 11 (A) Plexiform bone from a three-year-old ewe (original magnification $\mathrm{x}$ 25). (B) Haversian remodeling from the caudal femoral cortex of an eightyear-old ewe (original magnification $x$ 25)[33]

Figure 12 (Left) Three-dimensional reconstruction of a sheep vertebral body biopsy from the control group (Right) Three-dimensional reconstruction of a sheep vertebral body biopsy after 6 months of osteoporosis induction with ovariectomy, steroid application and a calcium/vitamin D-restricted diet [36]....29

Figure 13 Approximate anatomy of the radial-ulnar sectors divided into six sections as indicated by the gray lines. The top tight is the cranial aspect and the top left is the lateral aspect [37]. 32

Figure 14 Schematic of the microradiograph layout. 


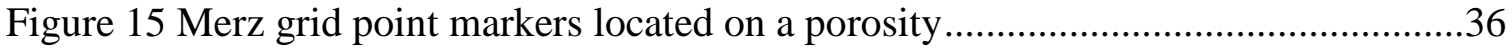

Figure 16 Merz grid points located on remodeled tissue .............................................36

Figure 17 Secondary osteons located within the scope of the Merz grid ..........................37

Figure 18 Use of the Merz grid to count the number of cement line interfaces on secondary osteons

Figure 19 Images of each level of an aluminum step wedge, increasing thickness from left to right, top to bottom $(0 \mathrm{~mm}, 0.02 \mathrm{~mm}, 0.04 \mathrm{~mm}, 0.06 \mathrm{~mm}, 0.08$ $\mathrm{mm}, 0.1 \mathrm{~mm}, 0.12 \mathrm{~mm}$, and $0.14 \mathrm{~mm}$ respectively)

Figure 20 Mean BV/TV values and standard deviations for each season of the OVX sheep

Figure 21 Mean percent of remodeled tissue values and standard deviations for each season of the OVX sheep

Figure 22 Anatomic variability of the OVX sheep in the percentage of tissue remodeled. The polar graph orients the values for each sector in their approximate anatomical location. The radial distance indicates the value of the remodeling parameter.

Figure 23 Anatomic variability of OVX sheep within season for the percentage of material remodeled. The polar graph orients the values for each sector in their approximate anatomical location. The radial distance indicates the value of the remodeling parameter.

Figure 24 Anatomic variability of OVX sheep within season for the number of tissue cement line interfaces. The polar graph orients the values for each sector in their approximate anatomical location. The radial distance indicates the value of the remodeling parameter.

Figure 25 Anatomic variability of OVX sheep within season for the number of material cement line interfaces. The polar graph orients the values for each sector in their approximate anatomical location. The radial distance indicates the value of the remodeling parameter.

Figure 26 Mean BV/TV and standard deviations for control and OVX sheep for each season.

Figure 27 Mean percentage of tissue remodeled and standard deviations for control and OVX sheep for each season.

Figure 28 Anatomic variability in the percentage of tissue remodeled for control and OVX sheep. The polar graph orients the values for each sector in their approximate anatomical location. The radial distance indicates the value of the remodeling parameter.

Figure 29 Mean percentage of remodeled material and standard deviations for control and OVX sheep for each season.

Figure 30 Anatomic variability in the percentage of material remodeled for control and OVX sheep. The polar graph orients the values for each sector in their 
approximate anatomical location. The radial distance indicates the value of the remodeling parameter. .............................................................................56

Figure 31 Mean secondary osteon radius (um) and standard deviations for control

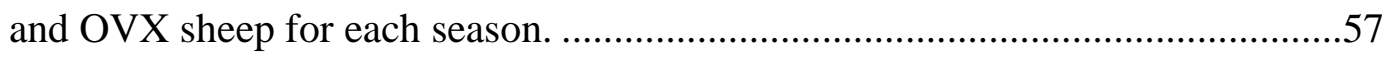

Figure 32 Mean number of tissue cement line interfaces and standard deviations for control and OVX sheep for each season.

Figure 33 Mean number of material cement line interfaces and standard deviations for control and OVX sheep for each season.

Figure 34 Anatomic variability in density measured in equivalent thickness of aluminum (ETA) for OVX sheep. The polar graph orients the values for each sector in their approximate anatomical location. The radial distance indicates the value of the remodeling parameter.

Figure 35 Images of the tibia (left) and radius (right). A. View demonstrating the reference line (solid line) and the measurement site (between dotted lines); B-E, images from a premenopausal (B), postmenopausal osteopenic (C), postmenopausal osteoporotic (D), and postmenopausal severe osteoporotic (E) subject. [48] 


\section{INTRODUCTION}

\subsection{Purpose}

Osteoporosis is a major health threat for an estimated 44 million Americans or 55 percent of people 50 years of age or older [1]. In the U.S. today, 10 million individuals already have the disease with 8 million of them being women [1]. According to the World Health Organization, osteoporosis is defined on the basis of bone mineral density (BMD) assessment using specific criteria. A BMD that lies 2.5 standard deviations or more below the average value for a healthy individual is considered to be osteoporosis [2]. Osteoporosis involves a decrease in bone mass, and an alteration of microarchitecture leading to increased bone fragility. Increased fracture risk with low-energy trauma and age has proven to be the major complication of osteoporosis [3]. Osteoporosis frequently goes undetected until the bone fractures, with fractures commonly occurring in the spine, hip, wrist, humerus, and pelvis [4]. An understanding of the mechanism behind osteoporosis is necessary to improve therapeutic agents and the animal models required to test them. However, a discussion on the anatomy of bone must first be addressed in order to fully understand osteoporosis.

\subsection{Skeletal Biomechanics}

\subsubsection{Bone Function and Structure}

Bone provides a framework to support the body, protection of vital organs, an attachment site for muscles to allow for movement, a reservoir for calcium homeostasis, and blood cell formation [5]. As a highly dynamic tissue, bone is continually changing to its physiologic and mechanical environment. These changes in the environment impart energy to the bone [6]. Due to bone's flexibility, it has the ability to conform to the 
absorbed energy. Bone is a unique material in that it is able to achieve stiffness while still remaining flexible, and strength while still maintaining lightness through its material composition and structural design [6].

Four cellular components are essential in bone's ability to support and maintain the body. Osteoclasts, multinucleated cells, are responsible for bone resorption [7]. Formed by the fusion of monocytes in the bone marrow, osteoclasts form a brush or ruffle border around the cell, allowing for resorption. Mononuclear, cuboidal cells called osteoblasts produce the new layers of bone and are derived from mesenchymal cells [7]. Osteocytes are former osteoblasts that have become buried in the bone and are in an active state within cavities called lacunae. Osteocytes have processes that allow them to form connections with the other bone cells [7]. The pores found in bone are interconnected through canaliculi, tying all the osteocytes together. This allows nutrients to reach the osteocytes by diffusing through the canaliculi [5]. Bone lining cells are also former osteoblasts. However, bone lining cells do not get buried in the bone matrix and instead line all bone surfaces. These cellular components play a key role in the remodeling of bone and will be discussed in a later section [7].

Bone surfaces are covered by two membranes: the periosteum and the endosteum. Aside from the joint surfaces, the external surface of the entire bone is composed of double-layered membrane called the periosteum. The outer layer is dense fibrous connective tissue while the inner layer consists primarily of osteoblasts and osteoclasts. The periosteum is richly supplied with nerve fibers, lymphatic vessels, and blood vessels, providing an insertion point for tendons and ligaments. Internal bone surfaces are covered 
by the endosteal surface, a delicate connective tissue membrane that covers the trabeculae and lines the canals of cortical bone [5].

Cortical and trabecular bone are the two types of bone tissue. Cortical bone, also known as compact bone, is the dense bone that can be found in the shafts of long bones and on the outer layer of bone. The porosity of this type of bone ranges from 5 to 10 percent [7]. The main structural unit of cortical bone is the osteon, an elongated cylinder generally oriented parallel to the long axis of the bone that acts as load-bearing pillars as seen in Figure 1 [5]. Due to its stiffness, cortical bone is responsible for bearing most of the load from the body. Trabecular or cancellous bone is composed of thin plates, or trabeculae, in a loose mesh structure [8]. Trabecular bone is much more porous than cortical bone, with a porosity ranging from 75 to 90 percent. Trabecular bone can be found in the vertebrae, flat bones, and in the end of long bones [5]. 


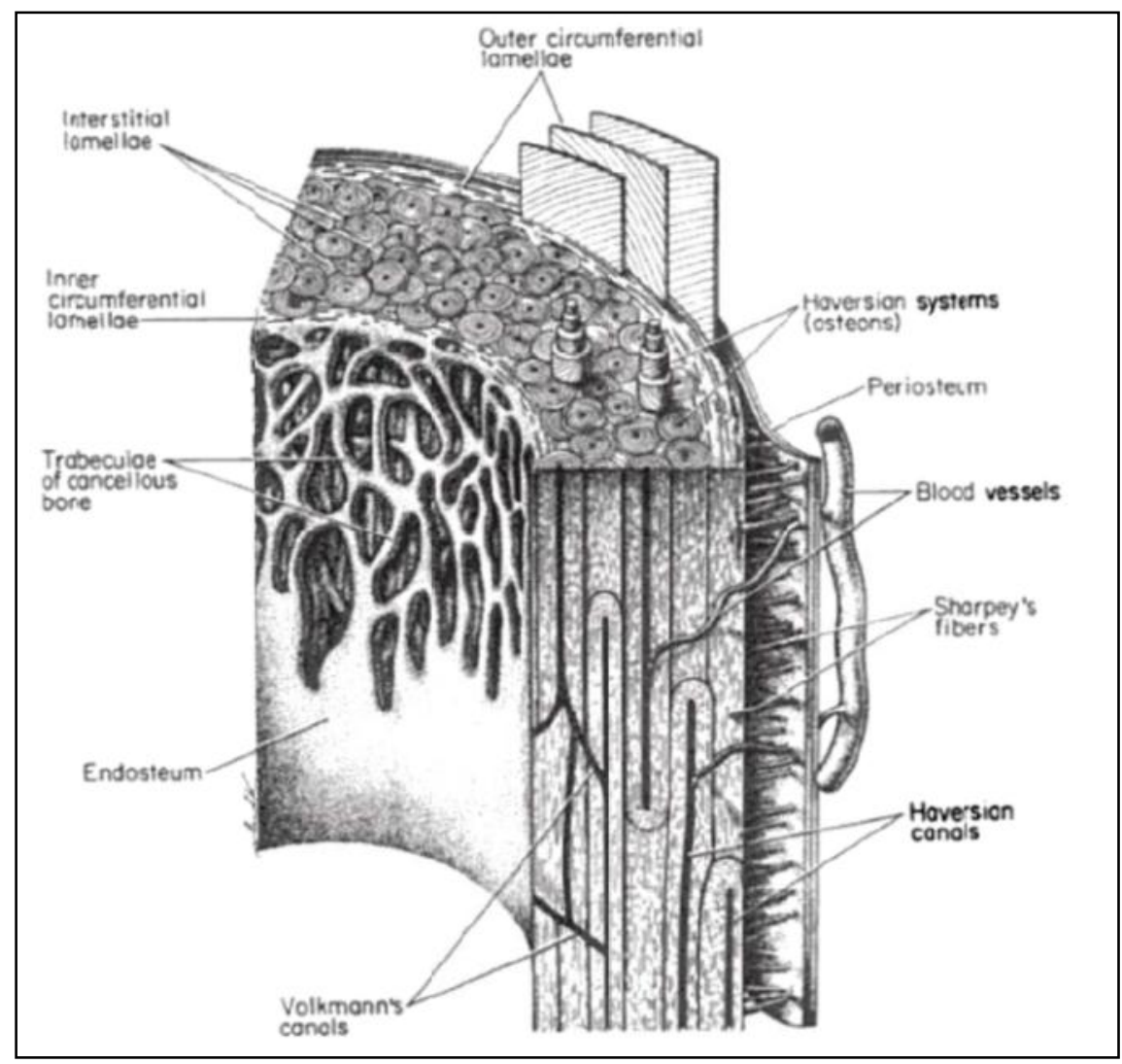

Figure 1 Bone structure for cortical and trabecular bone [7]

The primary sources of porosity found in cortical bone are Haversian canals, Volkmann's canals, and resorption cavities. Haversian canals are found in the center of osteons and usually aligned to the long axis of bone. They contain capillaries and nerves that are necessary for providing nutrients to the bone. The size of Haversian canals are estimated to be $50 \mu \mathrm{m}$ in diameter, approximately the diameter of a human hair [7]. Volkmann's canals, short and transverse canals, connect the Haversian canals to each other and the outside surfaces of the bone. Resorption cavities represent voids that have been created by the osteoclasts. These spaces are typically temporary, do not contain any bone formation, however bone formation sometimes can be seen, and are the initial stages of remodeling, a process that will be discussed in a later section [7]. 
Compact bone can be classified as either primary or secondary bone. Primary bone is the first bone formed on an existing bone surface during growth. Primary bone can be of two types: circumferential lamellar bone or plexiform bone. Lamellar bone forms slowly in a highly organized manner and is composed of parallel layers [7]. In circumferential lamellar bone, the lamellae are parallel to the bone surface. The formation of primary osteons occurs through blood vessels incorporating into the parallel layers until the vessel is completely surrounded by several lamellae. Plexiform bone formation occurs at a much quicker rate and involves continually constructing a trabecular network on the surface and filling in the gaps, resulting in a mixture of woven bone and lamellar bone [7]. Based on the shape of its vascular spaces, plexiform bone has a "brick wall" appearance. Plexiform is rarely seen in humans and is most typically seen in large, fast growing animals such as ungulates. Through remodeling, the resorption of preexisting bone and its replacement with new, lamellar bone results in secondary bone. In compact bone, secondary bone consists of secondary osteons that are about $200 \mu \mathrm{m}$ in diameter and centered around a Haversian canal [7]. The boundary that surrounds the secondary osteon is known as the cement line. Like cortical bone, trabecular bone is remodeled in a similar manner. The main difference is the remodeling of trabecular bone rarely produces osteons because they usually do not fit inside the individual trabeculae. As a result, in the place of an osteonal canal a bone marrow space will form [7]. In adult humans, most of the cortical bone and trabecular bone is composed of secondary bone. This is due to the constant turnover of bone throughout one's lifetime [7]. 


\subsubsection{Material Composition of Bone}

Bone is a two-phase compound with both organic and inorganic components. It is different than the other tissues in the body in that it is largely mineral or inorganic, 60 to 70 percent of its dry weight [8]. The minerals consist primarily of calcium and phosphate in the form of hydroxyapatite crystals, giving bone its solid consistency. The organic matrix is largely Type I collagen fibers and ground substance. Collagen plays a critical role in the structure and function of bone tissue; the fibers are tough and pliable, yet they resist stretching and have little extensibility $[8,9]$. Other components of the organic matrix include proteoglycans and noncollagenous proteins. The relative amounts of each component of bone contribute to the overall strength of bone.

The structural organization of bone contributes to its mechanical properties. Cortical bone and trabecular bone have significantly different mechanical properties. Cortical bone is an anisotropic material, exhibiting a highly ordered arrangement of collagen fibers and mineral crystals. The longitudinal arrangement of collagen fibers and minerals make cortical bone stronger and stiffer in this direction [7]. It is much stronger in compression when loaded longitudinally as opposed to when loaded transversely.

Also, osteonal remodeling tends to convert cortical bone from an orthotropic material to a transversely isotropic material [7].

Trabecular bone is substantially weaker and much more compliant than cortical bone. This is because trabecular bone is a porous material and its mechanical properties are governed by the magnitude of the porosity. In addition to porosity, the properties of trabecular bone are also determined by the anisotropy of the trabecular architecture, and 
the material properties of the tissue within the individual trabeculae [7]. However, for the purpose of this experiment only cortical bone is of interest.

Osteons play a critical role in bone's response to a mechanical stimulus; however other factors have an effect. Porosity, the ratio of void volume to total volume, affects both cortical and trabecular bone. In cortical bone, Haversian canals and Volkmann's canals affect the mechanical properties. The bone marrow spaces between the trabeculae affect the properties of trabecular bone [7]. Several researchers have proposed relationships between the mechanical properties of bone and porosity. Figure 2 shows data compiled from several experiments exhibiting the relationship between porosity and strength. There is an initial linear relationship with small increases in porosity dramatically affecting bone strength. However, when the porosity is 0.1 the relationship changes and there is a more gradual decline in the ultimate stress in response to further changes in porosity [7]. 


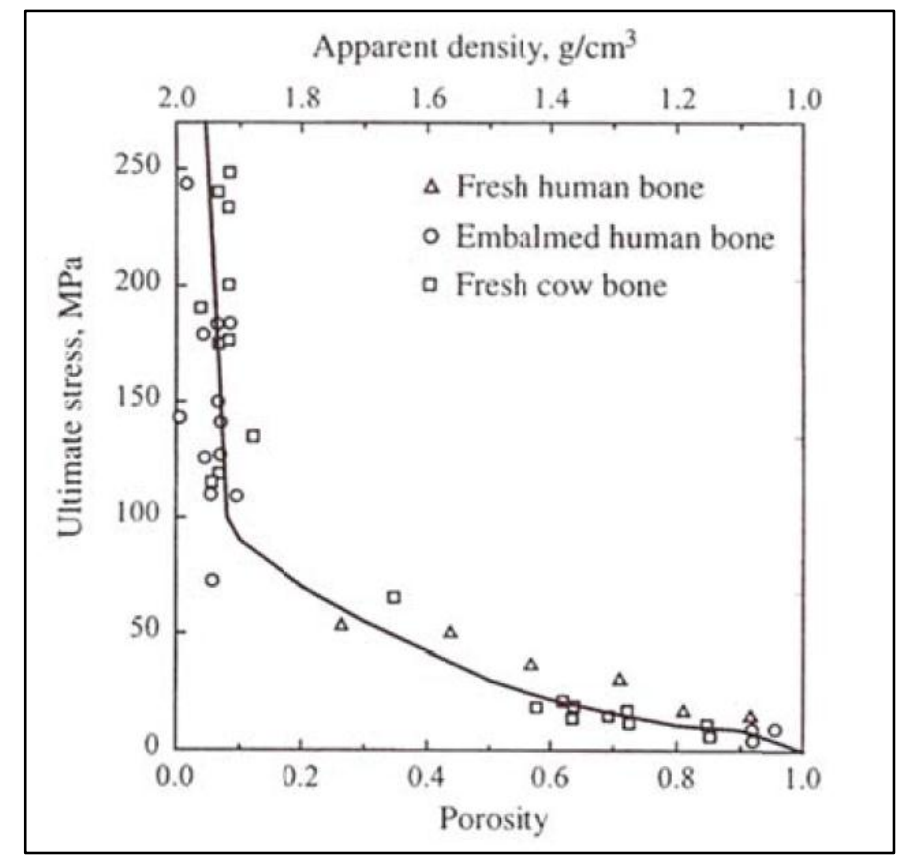

Figure 2 The relationships between ultimate compress stress, porosity, and apparent density for fresh human bone (triangles), embalmed human bone (circles), and fresh bovine bone (squares) [7]

Variations in the mineral content of bone also play a role in its mechanical properties. The amount of mineral content is based on the degree and cause of mineralization. Bone formed at a higher rate consequently has reduced mineral content. This can be related to age, trauma, or metabolic disease. Reduced mineral content can also be caused by a delay in the complete formation of new osteons. Vose and Kubala conducted a study correlating strength and density, finding that a small difference in mineralization made large differences in breaking stress and found that the point of maximum toughness occurred when bone was 66 to 67 percent mineralized [7].

When considering the material properties of bone, density always has an effect. Density refers to the specific gravity of the solid matrix. While the apparent density represents the mass per unit volume of a region of bulk bone, including the bone voids 
[7]. Depending on the area of interest, apparent density can be a much more useful property as it is a function of the porosity and mineralization.

\subsubsection{Quantitative Analysis of Skeletal Biomechanics}

The ability to quantitatively assess the skeletal system and more importantly prevent fractures from occurring is of utmost importance. Analyzing bone structure and bone mass can be performed both invasively and non-invasively. Micro computed tomographic scanning has been adopted as a method to measure bone microarchitecture. It has the ability to image small specimens of bone in three dimensions without destroying the tissue sample [10]. Other methods exist that allow for noninvasive measurement of bone mass. Most of the methods depend on the absorption of photons by the bone, and because minerals readily absorbs photons the amount of bone mineral can be measured [7]. Dual energy x-ray absorptiometry (DEXA) was first used clinically in the 1990's and involves low-energy X-rays scanning back and forth along the bone to produce a two-dimensional image. These methods are accurate to within 1 to 2 percent of the patient's actual bone mineral content and exposes the patient to far less radiation then what would occur if a standard X-ray were used [7].

Stereology is the method for analyzing a three-dimensional space when only twodimensional sections or their projections onto a surface are available. It is extremely useful in determining variables related to the structure and composition of bone [7]. Stereology involves taking histologic sections of the specimen and then analyzing them under a microscope. Several methods have been developed for measuring the porosity of the image. One method places a grid of points over the image and the number of points that falls on a void allows the user to find the percentage of porosity for that section [7]. 
Different types of grids have developed for this purpose. Grids that contain micrometers can be used to directly measure osteon diameters. A Merz grid is one example of a type of grid that can be used (Figure 3). It consists of 36 point markers and 6 sinusoidal lines. Typically, the Merz grid is superimposed onto an image through either an eyepiece reticule or by physically placing the grid onto an enlarged image.

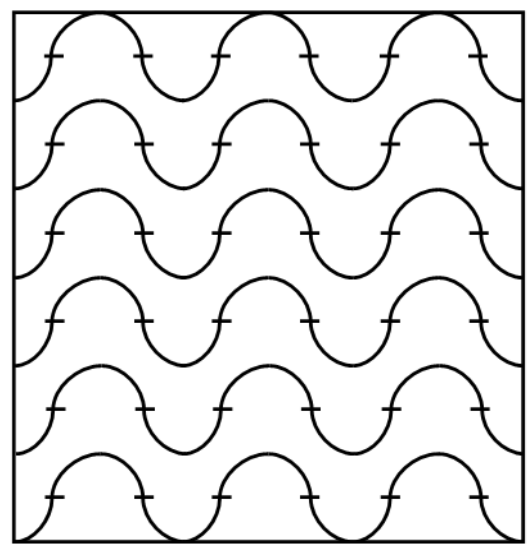

Figure 3 Merz grid with point markers and sinusoidal lines

\subsection{Bone Remodeling}

The cellular mechanisms of modeling and remodeling are responsible for bone adaptations. In modeling and remodeling, the removal and addition of bone occurs through the same cellular components. However, the goals of the two processes are entirely different. The cellular components involved are the osteoclasts, cells that remove bone, and the osteoblasts, cells that add bone.

Modeling is vigorous during growth and produces a change in the size and shape of bone. This occurs when new bone is deposited by the osteoblasts without previous bone resorption [11]. Modeling involves independent actions of the osteoclasts and osteoblasts. Due to the fact that modeling occurs primarily during growth, the rate of modeling is greatly reduced after reaching skeletal maturity. At a particular site, 
modeling is a continuous and prolonged process that is essential for adaptation of the body during growth and new loading occurs on the skeleton [7].

Remodeling occurs throughout one's life; however like modeling it is reduced after growth stops. It involves coupled actions by the osteoblasts and osteoclasts and does not typically affect the size and shape of bones. Each occurrence of remodeling is considered to have a definite beginning and ending. This process is essential for removing old and damaged bone and replacing it with new bone (Figure 4).

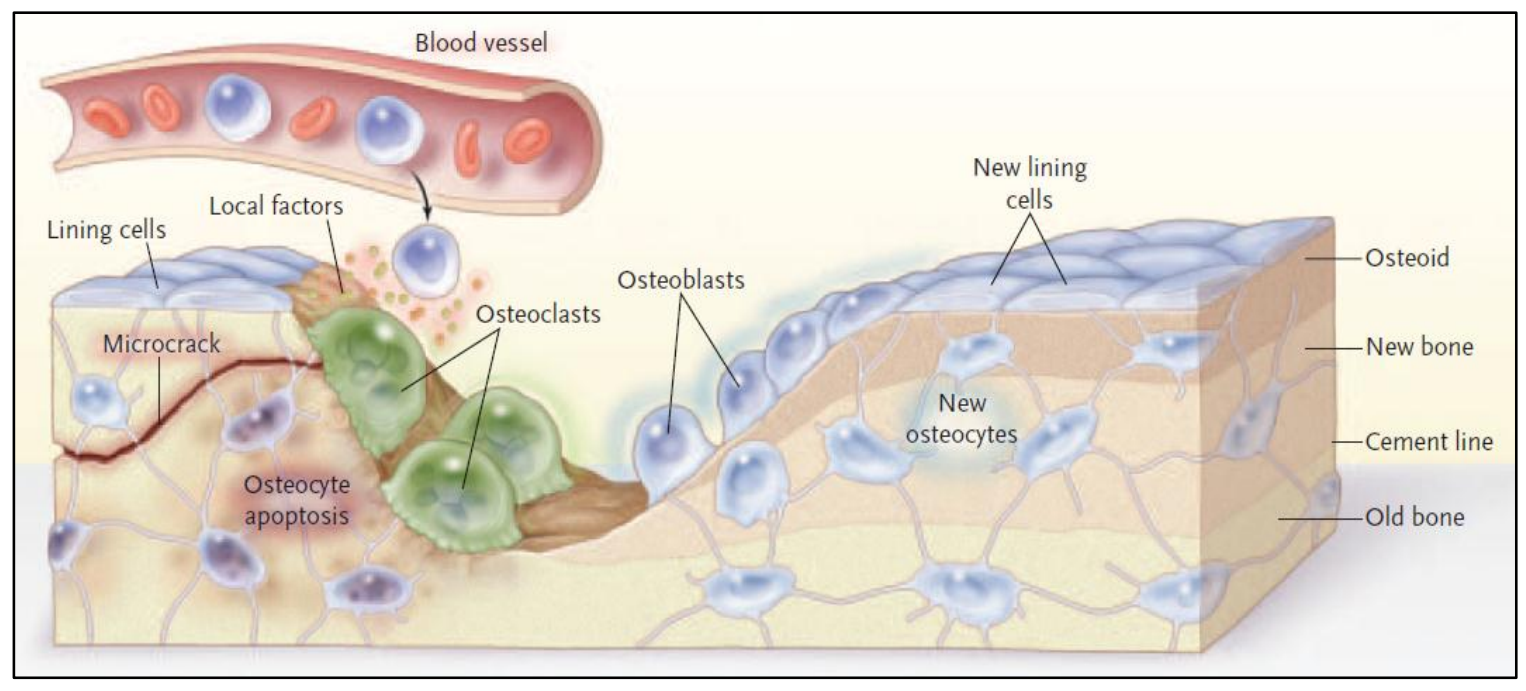

Figure 4 The Remodeling Cycle on a Trabeculum [11]

\subsubsection{Remodeling: BMUs and ARF}

Bone remodeling is an active process throughout the skeleton, essential for maintenance and renewal of the skeleton in adults. It is responsible for the removal of damaged bone and the subsequent formation phase restoring the structure of the bone [11]. Remodeling prevents accumulation of fatigue damage that could potentially lead to fatigue fracture [7]. Bone is remodeled through the coupled removal of bone and its replacement through the synthesis of a new bone matrix and its subsequent mineralization [12]. This process occurs through the teaming of osteoclasts and osteoblasts working 
together in basic multicellular units, or BMUs. A BMU typically consists of about 10 osteoclasts and several hundred osteoblasts [7].

A BMU's lifetime consists of three principal stages: activation, resorption, and formation (ARF). During activation, a chemical or mechanical signal brings about the formation of osteoclasts through the fusion of monocytes. The signal initiation will be further discussed in a later section. The osteoclasts then remove a volume of bone in either the form of a ditch or a tunnel. The tunnel made by the osteoclasts will be about $200 \mu \mathrm{m}$ in diameter [7]. During this tunneling, as seen in Figure 5, a resorption space is created to ultimately allow for the formation of a secondary osteon. The cutting cone creates the resorption area that occurs deep within the cortex of bone. To maintain nutritional access to the new bone, a new local vascular supply must be established. Thus, when the osteoblasts replace the resorbed bone, they do not completely fill in the tunnel. A Haversian canal is left in order to allow a constant blood supply to the newly formed osteon. The laying down of bone by the osteoblasts makes up the formation stage of the ARF cycle. The formation period is much slower than the resorption, with the total remodeling period taking 4 months [7]. 


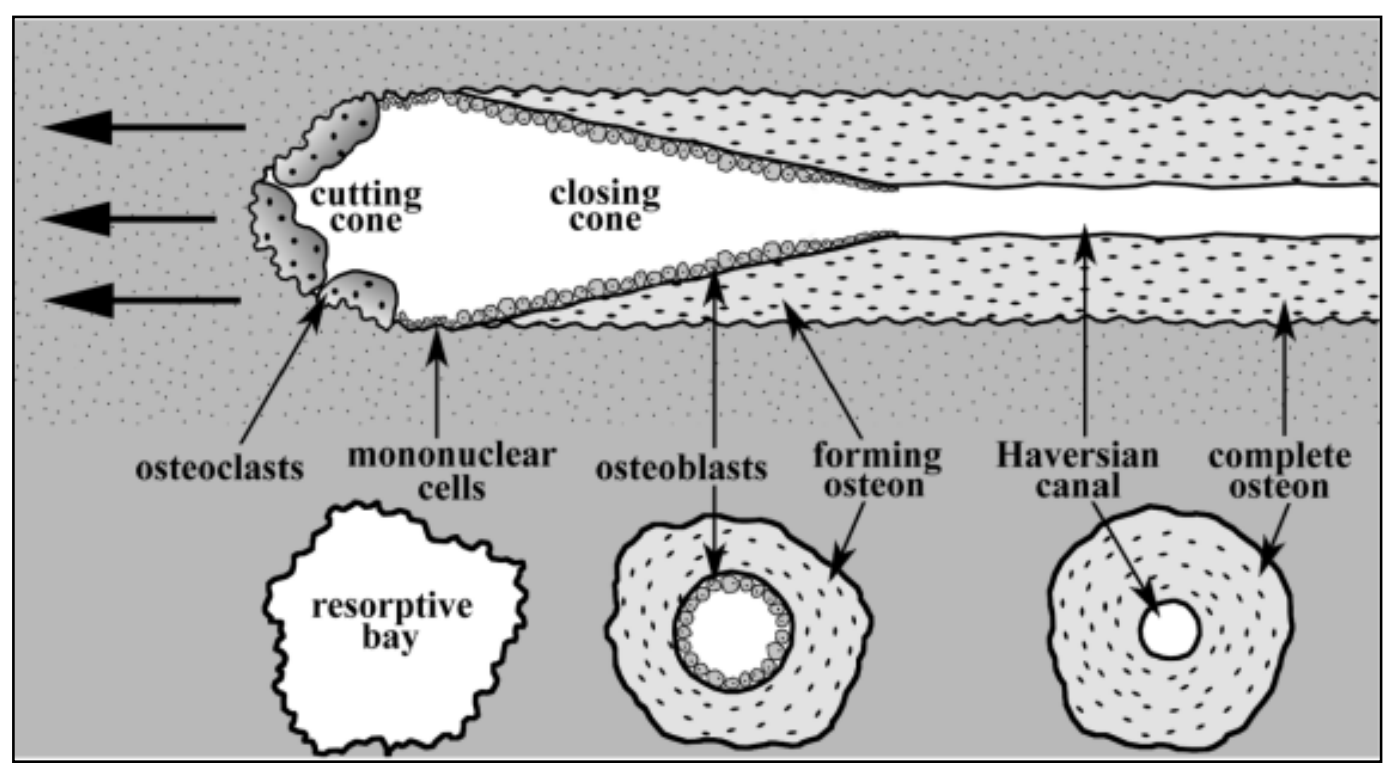

Figure 5 Tunneling of osteoclasts to form a new osteon [13]

The ARF cycle can be further broken down into 6 sequential stages of bone remodeling: activation, resorption, reversal, formation, mineralization, and quiescence [7]. Activation does not include the initial recruitment of the osteoclasts and typically occurs over 3 days. After activation, the osteoclasts can begin resorbing bone. They resorb at a rate of $40 \mu \mathrm{m} /$ day, moving longitudinally creating the cutting cone shown in Figure 5 [7]. There is not an immediate switch from osteoclast to osteoblast activity, it can take several days. A transition phase known as reversal occurs, resulting in a cylindrical space between the resorption and refilling regions. The line surrounding the reversal area is known as the cement line or reversal line. The combined reversal and resorption period in humans takes about 30 days [7]. During formation, the osteoblasts lay down concentric lamellae at an average radial closure rate of 1 to $2 \mu \mathrm{m} /$ day [7]. To allow for vascularization, the osteoblasts do not completely refill the tunnel, but leave a Haversian canal of 40 to $50 \mu \mathrm{m}$ in diameter. In humans, the formation phase averages about 3 months [7]. Following deposition of the new bone, mineralization of the organic 
matrix occurs within and between the collagen fibers. During the first few day of mineralization, 60 percent of the mineral content is laid down, referred as primary mineralization. Secondary mineralization occurs when the remaining 40 percent of the mineral content is laid down at a decreasing rate for 6 months [7]. The resorption of old, dense bone and its subsequent replacement with new, low-density bone brings about a change in the mechanical properties in the osteons and surrounding tissues. In the final phase of quiescence, the osteoclasts are no longer present and the osteoblasts either become osteocytes, bone lining cells in the Haversian canal, or disappear via apoptosis [7].

\subsubsection{Signaling and Initiation of Bone Remodeling}

The exact mechanism behind initiation of bone remodeling is not clearly understood. However, many researchers have published their theories on the initiation of bone modeling along with supporting scientific evidence. The idea of bone remodeling itself was popularized by Wolff [14]. Wolff wrote, "Thus the law of bone remodeling is the law according to which alterations of the internal architecture clearly observed and following mathematical rules, as well as secondary alterations of the external forms of the bones following the same mathematical rules, occur as a consequence of primary changes in the shape and stressing or in the stressing of the bones" [15]. His law focuses on the concept that the self-regulation of bone structures by cells is in response to a mechanical stimulus [7]. Investigators have developed theoretical models to quantify the relationship between mechanical loading and bone morphology. The backbone of the theories are founded in Harold Frost's Mechanostat theory which states that if bone tissue experiences excess mechanical stimulation, additional bone will be deposited. However, if bone tissue 
experiences insufficient mechanical stimulation, bone will be resorbed [16]. Other researchers claim that osteocytes are the key component in the remodeling cycle and act as the bone's mechanosensing cells. The osteocytes have the ability to sense strain on bone surfaces through stretch-activated ion channels, flow of interstitial fluid, and electrical potentials [14]. Additional research has been performed claiming that osteocytes that undergo apoptosis produce a signal initiating and guiding osteoclastic resorption of bone [17]. Osteocytes also have been suggested to sense fatigue damage and transmit signals activating remodeling in order to remove the damage [14]. Clearly, the theory behind bone remodeling is a controversial issue, with researchers each proposing their own mechanisms. Martin proposed a unifying theory for the control of bone remodeling based on five fundamental assumptions from other researchers as well as his own work [14]. His model first claims that bone lining cells are responsible for activating bone remodeling. Second, the network of osteocytes produces and transmits a signal proportional to the amount of mechanical loading. Third, osteocytes send an inhibitory signal to osteoblasts that reduces their rate of bone production. In summary, Martin argues that the signal produced by the osteocytes is in response to mechanical loading, and inhibits bone lining cells from activating remodeling [14]. The theories presented apply only to remodeling; however the signal generated by the osteocytes may also help control the modeling process.

\subsubsection{Quantitative Analysis of Bone Remodeling}

Analysis of histologic sections of bone provides a means for quantifying bone remodeling. Frost was the first to demonstrate this in the cortex of the human rib [7]. Today, the method has been adapted and applied to bone biopsies from the iliac crest. 
The use of histomorphometric analysis requires the reduction of the mechanics of remodeling and bone formation to definitive steps for analysis. Additionally, it provides a useful method for identifying inconsistencies in theories and problems associated with diagnosing pathologic states and defining their etiology [7].

The methodology of Frost serves as the foundation for this field, but over time it has been updated with new technology for a much simpler approach. The method involves labeling bone with a vital fluorochrome stain for mineralizing bone such as tetracycline. The labels are given 7 to 14 days apart, with the second label ending at least 1 day before the bone specimen is taken [7]. The bone is cross sectioned to a thickness of $100 \mu \mathrm{m}$ and then stained to distinguish osteoid from mineralized bone and allows the labels to be seen. This stain is typically tetrachrome or osteochrome. The sections are best observed using epiflourescent light [7]. Figure 6 diagrams the stained osteons as seen through the microscope. The BMUs labeled " $\mathrm{R}$ " are undergoing resorption, while the completed osteons are indicated with a "C". BMUs that are still forming bone (F) have either a single label (F, S) or a double label (F, D) [7]. 


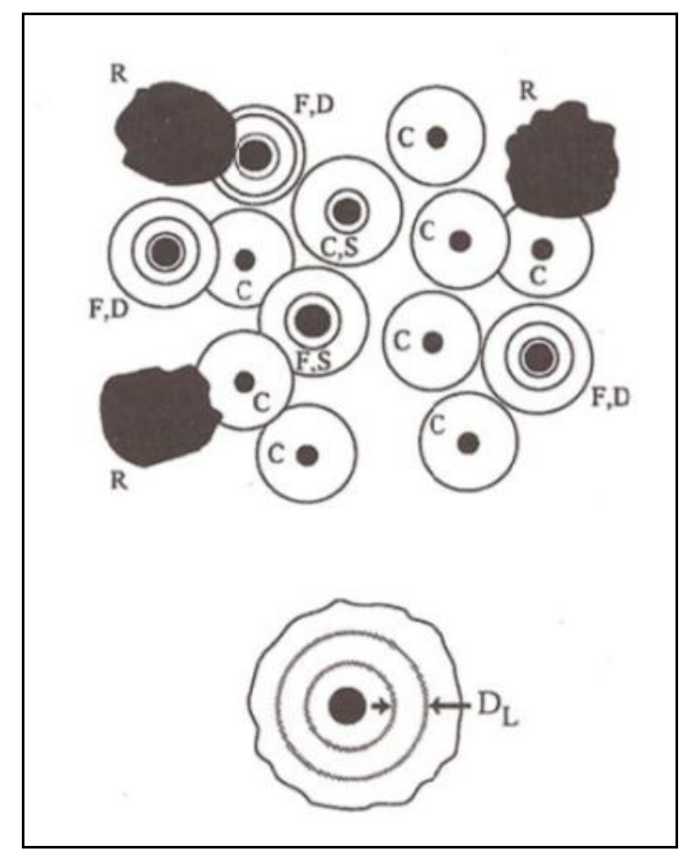

Figure 6 Upper: Schematic diagram of BMUs in a cross section of cortical bone. Black, irregularly shaped BMUs represent resorption spaces (R). BMUs with large canals are refilling; they have either a single label $(\mathrm{F}, \mathrm{S})$ or a double label $(\mathrm{F}, \mathrm{D})$. Completed osteons have small canals $($; ; some of these have a single label $(\mathrm{C}, \mathrm{S})$. Lower: A single BMU in the last stages of refilling is depicted. It has a double label; the distance between the labels is measured as shown $\left(\mathrm{D}_{\mathrm{L}}\right)$ [7].

Measurements are taken from the histologic sections in order to calculate the remodeling characteristics of the bone. The number of resorption spaces are identified; they have scalloped surfaces and lack of osteoid or fluorochrome label [7]. The perimeter of each resorption space is measured, and the mean perimeter is calculated. Then the number of refilling BMUs is determined, identified by their osteoid seams. The mean perimeter and thickness of the osteoid seams is found. From the double labeled BMUs, the mean distance $(\mu \mathrm{m})$ between the two labels can be determined. Finally, the number of completed osteons is recorded, indicated by the absence of osteoid seams [7]. The methodology of this study does not include staining. However, the number of secondary osteons as well as the number of cement line interfaces can be used in order to calculate the desired remodeling characteristics. 


\subsection{Osteoporosis}

Bone remodeling plays an important role in the development of osteoporosis.

Elderly patients, especially those at risk for osteoporosis, exhibit an imbalance between bone resorption and formation, particularly a negative balance. A negative balance occurs when the bone formation is less than the prior bone resorption, causing bone loss and compromising the strength of bone [11]. The micrographs shown in Figure 7 clearly depict the difference in microarchitecture between the 31 year old woman and 70 year old woman. The bone of the 70 year old is much more porous with an altered microarchitecture.

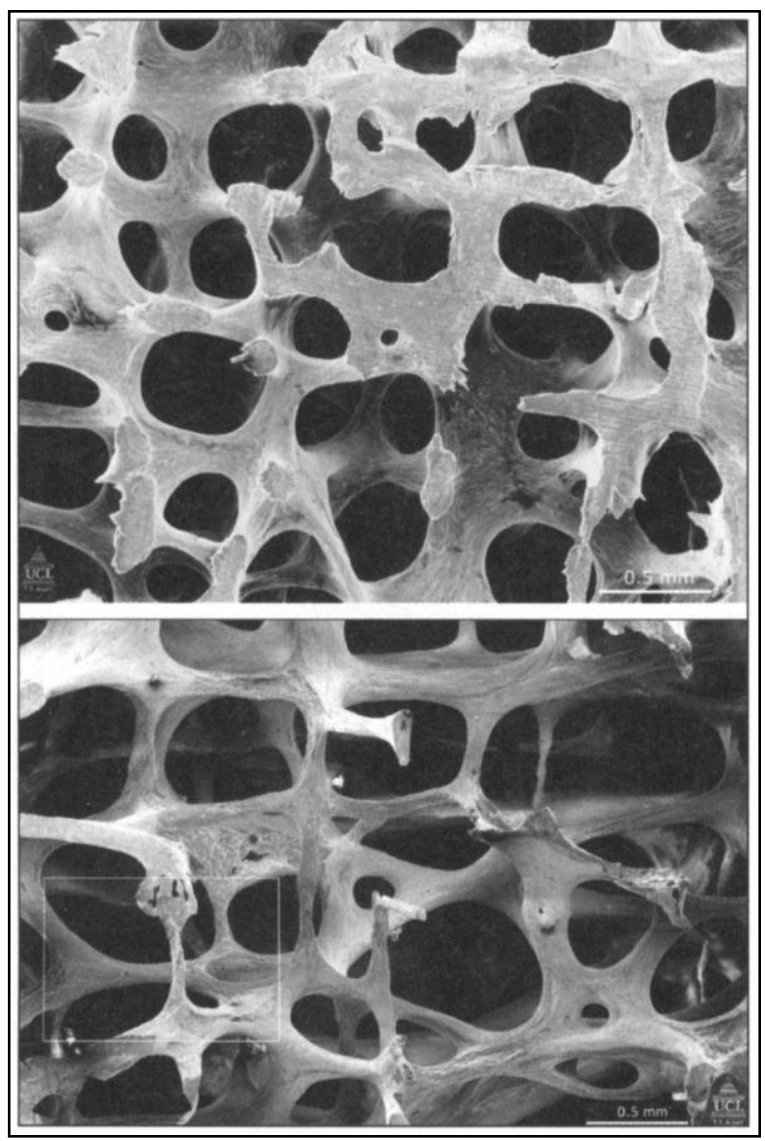

Figure 7 The structure of L3 vertebra in a 31 year old woman (top) and 70 year old woman (bottom) is shown using scanning electron micrographs. Note that many of the plate-like structures have become converted to thin rods [4] 
The effects of aging appear to be an important determinant for the onset of osteoporosis. With increasing age, individuals are more susceptible to fragility fractures after a minor traumatic event. Overall, bone mineral density (BMD) significantly falls during adulthood as a result of both age-related and menopause-related bone loss [18]. The roles of estrogen deficiency and age related bone loss in women has been well documented [4]. Menopause is the most important risk factor for bone loss in woman in late adulthood and as a result osteoporosis affects $30 \%$ of postmenopausal women [19, 20]. Thus, women are more susceptible to osteoporosis than men. The peak bone mass of men is 20 to 30 percent higher than women [18]. Other key factors for developing osteoporosis include calcium deficiency, immobilization, alcoholism, smoking and corticosteroid use [18]. Another possible contribution to the development of osteoporosis is the decreased production of insulin like growth factor from the liver. Genetic factors are believed to also have an influence on the peak bone mass, attained during the third decade of life. Peak bone mass is an important determinant of bone mass later in life and is influenced by nutritional intake and physical activity [4].

\subsubsection{Postmenopausal Osteoporosis}

During menopause, a woman's ovarian function declines and the ovaries no longer function as endocrine organs. As a result, the woman produces less estrogen and progesterone [5]. The decreasing levels of estrogen make postmenopausal women more susceptible to fracture incidence and developing osteoporosis. Bone loss early in the postmenopausal period contributes to increased fracture incidence with estrogen levels playing a key role in increased bone loss [21]. 
The mechanism by which estrogen induces bone loss is either through an increase in osteoclastogenesis or an increase in osteoclast lifespan. Estrogen acts by altering the cytokine dependency of the body and its cellular pathway can be seen in Figure 8. Monocytes and macrophages are responsible for producing interleukin-1 (IL-1) and tumor necrosis factor (TNF). Under normal conditions, estrogen inhibits the activity of IL-1 and TNF. However, in an estrogen deficient individual, this does not occur and IL-1 and TNF continue to stimulate proliferation of a group of mediating cytokines. These cytokines, receptor activator of NFkB ligand (RANKL), macrophage colony stimulating factor (M-CSF), granulocyte/monocyte-colony stimulating factor (GM-CSF), interleukin11 (IL-11), and interleukin-6 (IL-6), act directly on osteoclast progenitors to stimulate proliferation and inhibit apoptosis [22]. Additionally, IL-1 and TNF stimulate the synthesis of adhesion molecules, such as osteopontin, which contributes to increased cytokine production of IL-6. An important characteristic of the cytokines involved in regulation of osteoclastogenesis is their ability to stimulate their own as well as each other's synthesis (Figure 9) [22]. As previously stated, estrogen plays a role in bone loss by increasing osteoclast lifespan. Under normal conditions, all osteoclasts eventually die by apoptosis, a process stimulated through estrogen. Thus, in an individual who is estrogen deficient the osteoclasts do not undergo apoptosis as frequently, increasing the rate of remodeling and the volume of bone that is resorbed by prolonging the lifespan of osteoclasts $[11,22]$. 


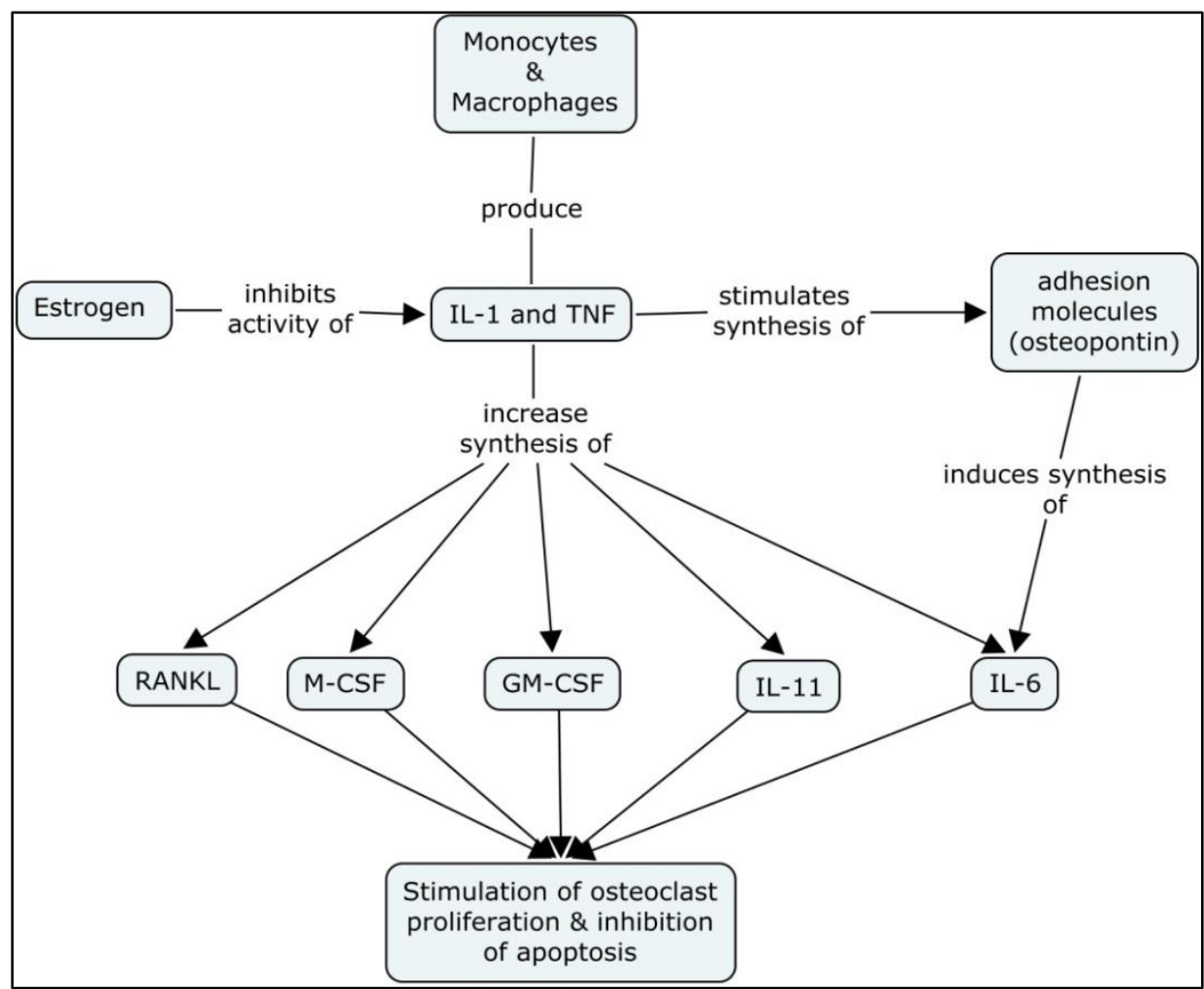

Figure 8 Cell signaling pathway occurring during estrogen deficiency

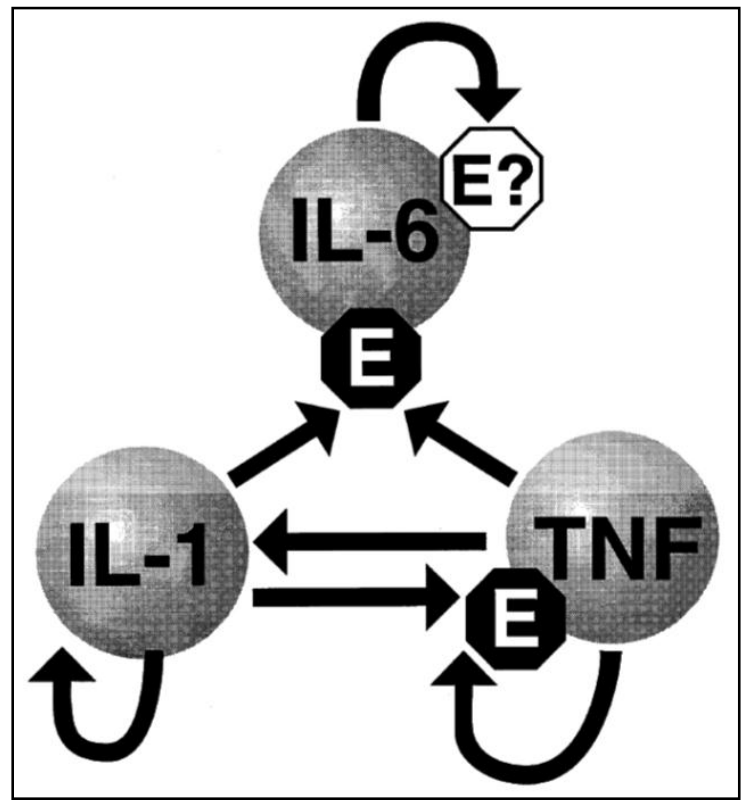

Figure 9 Regulation of IL-1, IL-6, and TNF production. Each arrow indicates a stimulatory effect of a cytokine on its own synthesis, or the synthesis of another cytokine. Demonstrated suppression of the stimulated synthesis of IL- 6 and TNF by estrogen ("E") is indicated by filled octagons, and the potential suppression of IL- 6 induced IL- 6 by estrogen is indicated by an open octagon [22] 


\subsubsection{Treatment Options for Osteoporosis}

Current treatment options for preventing or delaying the onset of osteoporosis as well as the fragility fractures associated with osteoporosis involve either increasing an individual's peak bone mass or preventing/slowing the rate of bone loss in later life. Nutrition supplements such as calcium and vitamin D can be given as treatments for osteoporosis. Calcium slows the rate of bones loss, especially in elderly women and in those with low calcium intake. It is generally prescribed in conjunction with other drugs for osteoporosis [23]. Vitamin D is also used as a treatment. Studies have shown trends for a reduction in non-vertebral fractures in elderly men and women treated with annual injections of calcium and vitamin D. However, the efficacy of calcium and vitamin D supplementation in healthy elderly people has yet to be established [23].

Hormone replacement therapy (HRT) is a therapy option not sought after due to the associated adverse effects. The decision to use HRT involves balancing the unknown risks of breast cancer, menstrual bleeding, and other possible gynecologic symptoms [18]. However, HRT can reduce markers of bone resorption, the rate of bone loss, and the incidence of fractures [24]. When initiated at the time of menopause, HRT is able to prevent bone loss. If the estrogen is administered in established osteoporosis, the treatment is less effective. This is because estrogens can aid in the replacement of only a small fraction of the lost bone mass [23]. Data from observational studies show a 40 to 60 percent reduction in fracture risk in women receiving HRT [24]. It has also been hypothesized that the use of calcium supplements could enhance the effects of estrogen on BMD. Even with evidence for HRT as an appropriate therapy for osteoporosis, a large 
portion of the population do not use it due to its contraindications or a perception of increased breast cancer [24].

Bisphosphonates are another type of treatment therapy for osteoporosis due to their high affinity for bone apatite. They are potent inhibitors of bone resorption, reducing the recruitment and activity of osteoclasts and increasing their apoptosis [23]. Long term treatment with bisphosphonates in human and nonhuman primates has shown increases in wall thickness, indicating an increase in osteoblast activity [25]. Bisphosphonates have also been studied in combination with other agents. Research has been done comparing the effects of bisphosphonates and HRT and suggests that both prevent bone loss relative to the controls [24]. Figure 10 diagrams the results from a 4year study comparing etidronate, a bisphosphonate, with and without HRT. The spinal BMD increased by 6.8 percent with just HRT, by 6.8 percent with etidronate, and by 10.9 percent using both HRT and etidronate. [24]. These results indicate a possibility for a similar mechanism for treatment using etidronate and HRT, with both affecting the activity of the osteoclasts. 


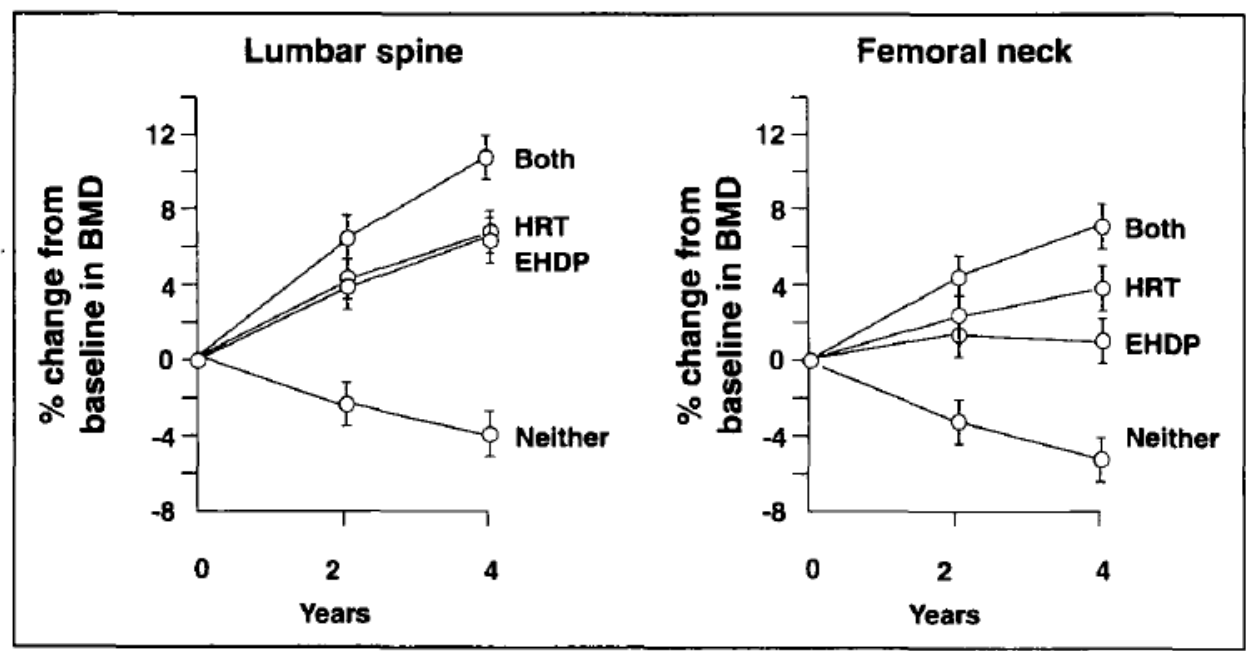

Figure 10 Lumbar spinal bone mineral density (BMD) increased similarly in estrogen and etidronate treated patients and by a greater amount using both. EHDP=etidronate; HRT=hormone replacement therapy [24]

\subsection{Necessity for Animal Models}

Before any medical device or pharmaceutical agent can be used in humans it must satisfy the safety and efficacy requirements set forth by the US Food and Drug Administration (FDA). This is shown through extensive bench and animal testing. Not only is animal testing used as a way to test potential therapies, but it also provides a means to better understand the progression of specific diseases. Animal testing typically begins with a smaller inexpensive model such as mice or rats and then progresses into larger diseased animals. The rationale for these procedures is to mitigate any risks and troubleshoot the treatment options in the smaller animals to minimize the time and costs. Thus, the more successful treatment option can then be placed in the larger and more physiologically relevant animal. Even with the more physiologically relevant animals, animal models cannot accurately simulate the differences amongst humans in nutrition, lifestyle, and physical activity, thus the use of human clinical trials is the next progression after the use of animals. 
Animal models involved in osteoporosis must be able to simulate the osteoporotic conditions in order to test drugs [26]. Additionally, the conditions should be optimal for testing surgical treatment of osteoporotic fractures and the design of prosthetics devices with different coatings to promote osseointegration. The devices must be able to perform and not loosen during the decreased bone mass as seen in osteoporosis [26].

Rodents, specifically rats, are the most commonly used animal for osteoporotic studies, presenting numerous advantages. They are inexpensive, easy to house, and the general public is accustomed to the use of rodents in research. Their short life span facilitates studies on the effects of aging on bone. Due to the rat's extensive use in research, much is known about bone turnover and the effect of diet on bone aging [27]. Cortical thinning and increased fragility are also well documented in aging rat and mice models. Rodents do not experience natural menopause, thus ovariectomy is used to produce an artificial menopause. The ovariectomized rat model exhibits most of the characteristics of human postmenopausal osteoporosis and has been used to study the effects of bisphosphonates on estrogen deficiency [28, 29]. However, in a rat model the development of Haversian systems and impaired osteoblast function during the late stages of menopause requires 3 to 4 months, making it a poor model for studying the effects of ovariectomy on cortical bone [29].

Rabbit models are not as commonly used to study the effects of ovarian insufficiency on bone mass. They are more frequently used to study bone ingrowth into implants and the bone-implant interface. Rabbits undergo Haversian remodeling, have rapid bone turnover, and show skeletal maturity at 7 to 8 months. Despite these advantages, rabbit models are not fully characterized for osteoporosis. Studies conducted 
using rabbits were only able to assess BMD and without an assessment of microarchitecture and bone mechanics a complete characterization of the rabbit model cannot be achieved [30].

Nonhuman primates demonstrate many advantages as they are physiologically relevant to humans. Their organ systems most closely resemble the human systems and include: the gastrointestinal tract, endocrine system, and bone metabolism. Baboon and macaques are the two most documented monkeys used. Female macaque monkeys cycle monthly and have hormonal patterns similar to humans. As a result, ovariectomy in these animals brings about significant reduction in vertebral cancellous volume. However, the female primate must be significantly aged to act as a model for postmenopausal women. Additionally, the use of primates is very costly, and they are difficult animals to handle to be used as a primary large animal model for osteoporosis [29].

Dogs have been used for studying the human skeleton because of their extensive BMU remodeling and larger size. Additionally, they are easy to work with and like humans they are monogastric. Unlike humans and the primates which are polyestrous, dogs are diestrus, with ovulation occurring twice a year. A clear disadvantage for dogs is removal of both ovaries and uteri doesn't appear to be sufficient to create significant bone loss [27]. Thus, the lack a sizeable response in histomorphometric, bone mass, and biochemical parameters limits the use of dogs in the study of postmenopausal osteoporosis [29].

\subsubsection{Proposed Animal Model}

A promising model for the study of osteoporosis is the skeletally mature sheep, more specifically ewes. Sheep present many advantages over other models. Its large size 
allows for device implantation and subsequent analysis. They are easy to manage and are available in large numbers, allowing for large scale studies [31]. The metabolic rate of sheep, based on the oxygen consumption per gram of body weight, is 0.22 , which is closer to that of humans (0.21) than to that of rats (0.87) or dogs (0.33) [32]. Another factor making the sheep an advantageous model is the hormone profile of ewes are temporally and quantitatively similar to those of women [32]. Young sheep have been shown to develop plexiform bone that is a combination of woven and lamellar bone. Additionally, 3 to 4 months after the development of plexiform bone, Haversian remodeling is visible in the cortical bone (Figure 11) [33].

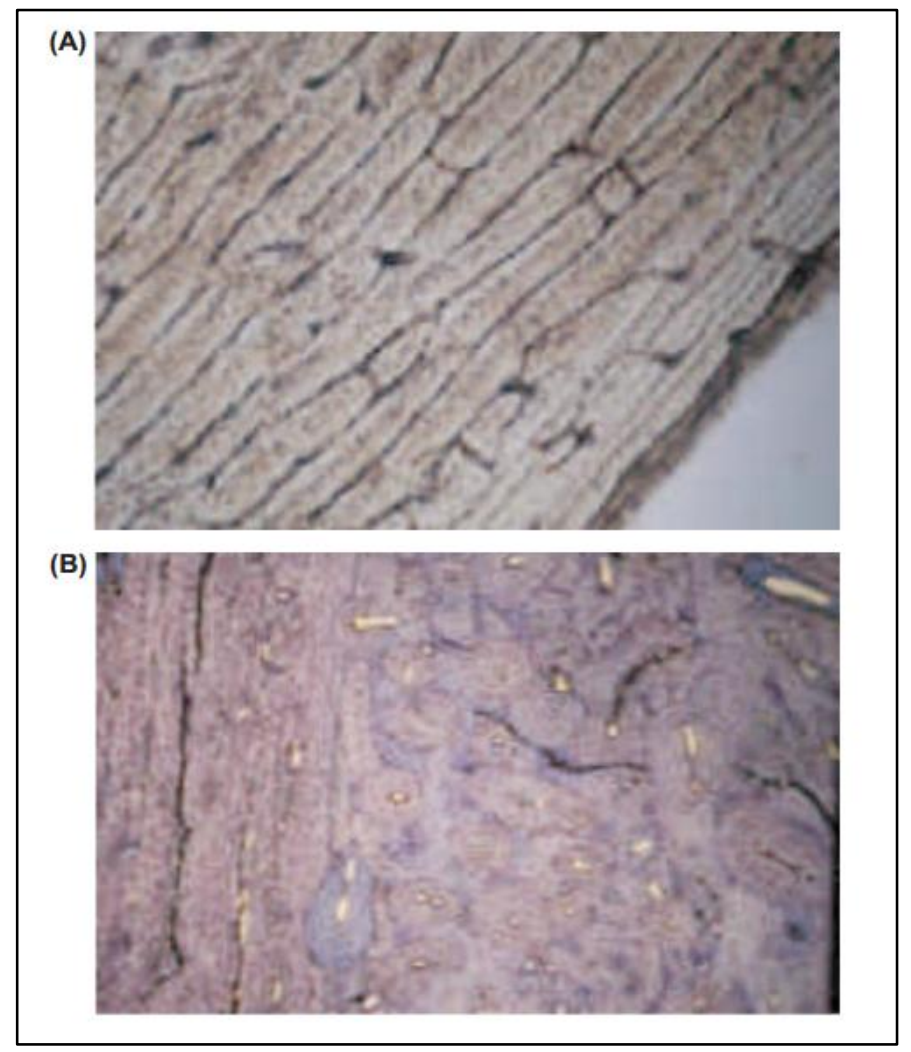

Figure 11 (A) Plexiform bone from a three-year-old ewe (original magnification x 25).

(B) Haversian remodeling from the caudal femoral cortex of an eight-year-old ewe (original magnification $\mathrm{x} 25$ )[33] 
Ewes have similar reproductive cycles to that of women. However, they do not have a clear menopause at midlife. Thus, they are not able to develop postmenopausal osteoporosis on their own making an ovariectomy necessary. The OVX sheep has been used as a model for loss of BMD. However, unlike female humans, ewes have an annual anestrous period of 1 to 2 months [32]. Another clear disadvantage for the use of sheep is their different gastrointestinal systems. In order to administer drugs orally a surgical insertion is required to bypass the microflora of the rumen [29].

When implementing the ewe as an animal model for postmenopausal osteoporosis, age and season of analysis are two factors that must be considered. An older ewe has been shown to better serve this purpose. Researchers have shown decreases in resorption surfaces, osteoblast surface, and bone formation rates when comparing biopsies of ewes 3 months apart. This supports the claim that changes in remodeling occur within 3 months of ovariectomy [34]. However, changes in bone mass has also been demonstrated in 3 to 4 year old ewes six months following ovariectomy (Figure 12) [34]. Variation does occur as other studies of osteoporosis did not see significant mechanical changes at the spine and the ilium until 12 to 24 months after ovariectomy [35]. 


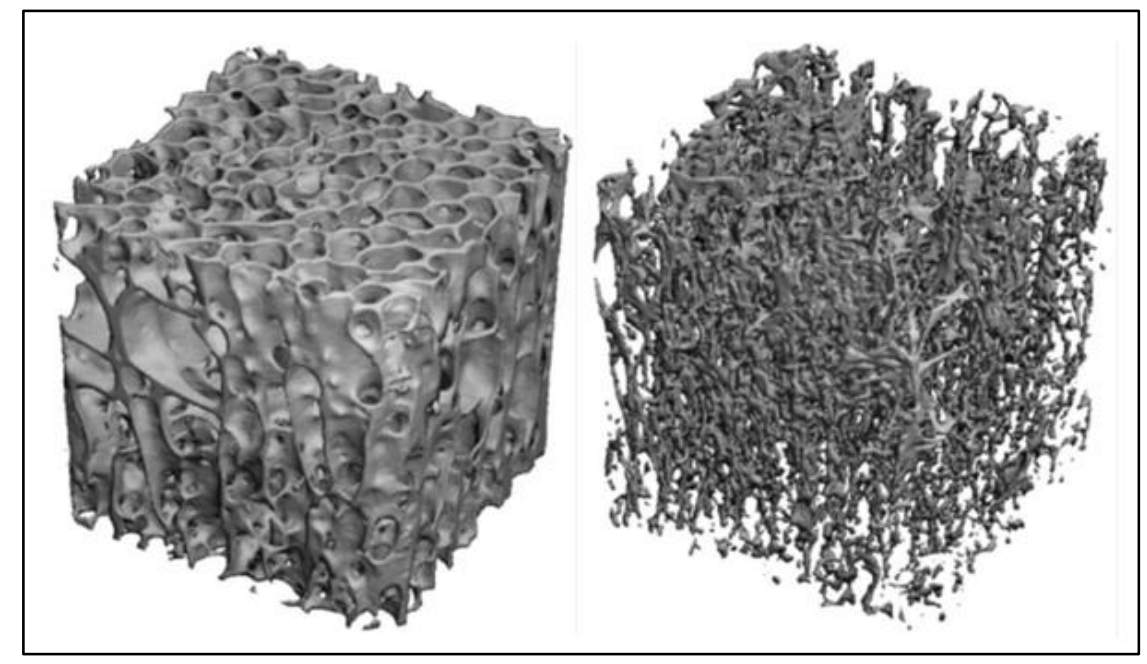

Figure 12 (Left) Three-dimensional reconstruction of a sheep vertebral body biopsy from the control group (Right) Three-dimensional reconstruction of a sheep vertebral body biopsy after 6 months of osteoporosis induction with ovariectomy, steroid application and a calcium/vitamin D-restricted diet [36]

Humans have been shown to have alteration in BMD due to seasonal variation, with the lowered BMD occurring in winter. This type of variation also occurs in sheep. The nutrition of the sheep directly influences the bone metabolism of the sheep, in particular calcium and vitamin D levels. Vitamin D is produced in the skin of the animals by ultraviolet (UV) radiation. Thus, the time period that the animals are exposed to light, and the daytime length or season of the year are important parameters to consider [31].

Overall, the ovariectomized ewe provides an adequate large model for postmenopausal osteoporosis. It allows for testing of therapeutic agents and prosthetic devices. The effects of seasonality, however, need further characterization. Through this research, a quantifiable model for the seasonal and anatomical variations in the bone remodeling will be determined from an ovariectomized animal and then further characterized through comparison to the control animal. 


\subsection{Study Objectives}

The purpose of this study is to quantify compact bone remodeling in adult ovine bone based on seasonal parameters. The amount of remodeling will be assessed using the following measurements: the ratio of bone volume to tissue volume, the percent of tissue remodeled, the percent of material remodeled, mean secondary osteonal radii, cement line interfaces as a function of tissue volume, cement line interfaces as a function of material volume, and bone mineral density. These measurements will be taken from the cortical bone of the radius and ulna of the ovine's left leg. Analysis will be made for variations across seasons, anatomical location, treatment, and a possible interaction among all three.

Research has been previously done showing that sheep do indeed undergo seasonal variations in bone properties [31]. However, little research has been done comparing the effect of anatomical location on remodeling parameters. It is expected that both seasonality and anatomical location will play a role in bone properties. In addition, a comparison will be made to sham operated sheep to see the effects of treatment. This model will determine the changes occurring based on season, anatomical location and treatment. The result of this study ensures future researchers are showing relevant information by using sheep as a model for postmenopausal osteoporosis. 


\section{MATERIALS AND METHODS}

\subsection{Animal Maintenance and Preparation}

As part of a larger experiment, 112 skeletally mature Columbia-Rambouillet cross ewes, 5 years or older, were acquired and kept at Colorado State University, Fort Collins, Colorado. This was performed under approval from the Animal Care and Use Committee. For the duration of the experiment, the animals were kept in dry lots at $41^{\circ}$ north and $1500 \mathrm{~m}$ altitude. Their diet consisted of a grass-alfalfa hay mixture. The 112 ewes were divided into four groups of 28 based on each season: autumn, winter, spring, and summer. These seasonal groups were then further divided into two groups of 14 . At the large animal surgery facilities at the College of Veterinary Medicine and Biomedical Sciences at Colorado State University, the groups were anesthetized and underwent surgery. Two types of surgeries were performed. One group of 14 underwent an ovariectomy (OVX) in which the ovaries were identified and subsequently removed. The other group of 14 served as the control group and underwent a sham surgery in which the ovaries were identified, handled, and not removed. The groups of 14 were then again divided into two groups. One group of 7 was sacrificed 3 months postoperatively while the other 7 were sacrificed 12 months postoperatively. The seasonal group represents the month that either the sham or OVX surgery was performed. Summer sheep underwent surgery in August. The autumn, winter, and spring sheep underwent surgery in November, February, and May respectively. One of the 12 month autumn sham sheep died prematurely and thus was included in the 3 month data as part of another study. The 12 month OVX animals in combination with the 12 month control animals for all seasons were the experimental subjects for this study. 


\subsection{Specimen Preparation}

After animal sacrifice, the sheep's right and left radius and ulna were removed. The bones were wrapped in a saline-soaked paper towel and stored in sealed plastic bags at a temperature of $-20^{\circ} \mathrm{C}$.The samples were then sent to Henry Ford Hospital. There, the samples were prepared for analysis. Using a band saw (Model 5212, Hobart Corporation, Troy, $\mathrm{OH}$ ) the center $50 \mathrm{~mm}$ of the diaphysis was removed. Then the radius was divided into six anatomical sectors using the Exakt cutting-grinding system (Exakt Corporation, Oklahoma City, OK). The six sectors were cranial, caudal, craniolateral, craniomedial, caudomedial, and caudolateral (Figure 13).

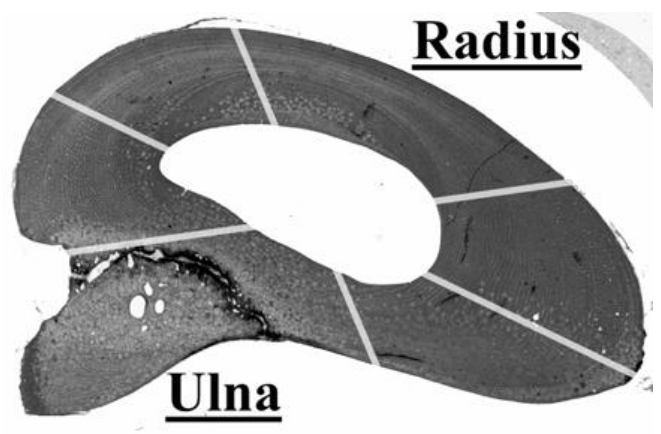

Figure 13 Approximate anatomy of the radial-ulnar sectors divided into six sections as indicated by the gray lines. The top tight is the cranial aspect and the top left is the lateral aspect [37].

From each of the anatomical sectors, $1.75 \times 1.75 \times 19 \mathrm{~mm}$ longitudinal cortical beams were obtained. In a separate study, dynamic mechanical testing was performed on the cortical beams from the left radii and ulnae. A $150 \mu \mathrm{m}$ section was cut out from the center of the left radii and ulnae beams and the remaining distal end was used for drying and ashing. This provided a method to determine density. The proximal section was frozen for an analysis to be performed at a later time.

To create microradiographs of the specimens, the $150 \mu \mathrm{m}$ sections were ground down by hand using fine grit sand paper to a final thickness of $100 \mu \mathrm{m}$. Using a 
2506AGHD 2.5x2.5x0.06 High Definition Photo emulsion Plates and an HP Cabinet Faxitron (HTA Enterprises, Microtome Technology Product, San Jose, CA) microradiographs were taken of each sections. This was done at $25 \mathrm{kV}$ for 20 minutes at 3mA. Each microradiograph contained sections from two sheep and an aluminum step wedge made with Reynolds Aluminum Foil in the middle (Figure 14). Aluminum Foil was chosen because it has an atomic number similar to the effective atomic number of hydroxyapatite [38]. By taping multiple layers of aluminum together in a stair step pattern, creating different levels of thickness, the aluminum step wedge was made. By taking the microradiographs of the aluminum and the specimens under the same conditions, we were able to standardize for exposure and development variation. The thickness of each step of aluminum is known and by comparing the specimen's image intensity to that of the aluminum step wedge a density measurement can be made relative to aluminum. 

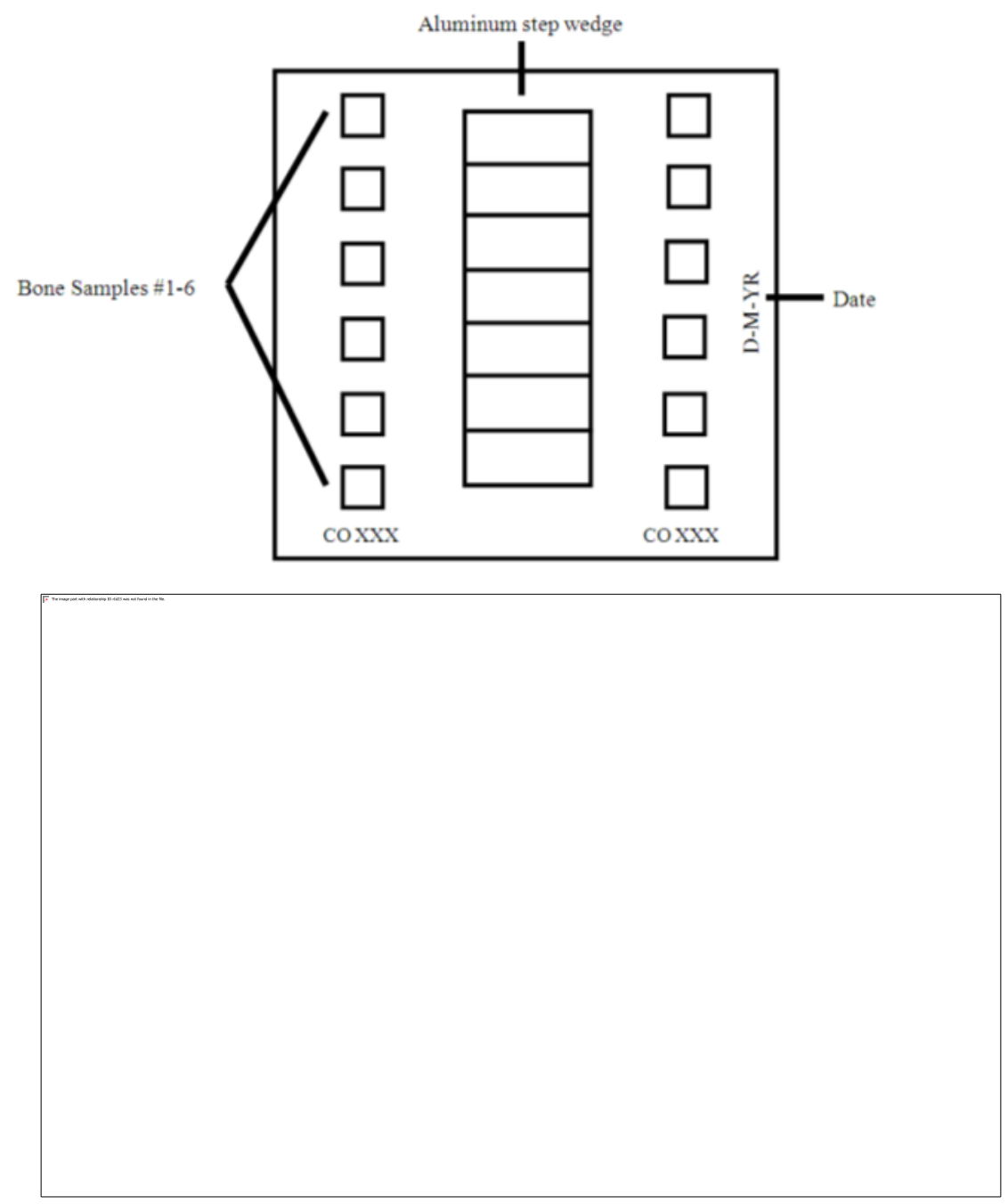

Figure 14 Schematic of the microradiograph layout

\subsection{Microradiograph Analysis}

The completed microradiographs were sent to California Polytechnic State University, San Luis Obispo from Henry Ford Hospital for analysis. The analysis included both histomorphometric and densitometry measurements. The histomorphometric measurements were performed to quantify the amount of remodeling occurring in the ovine bone. These measurements included: porosity, number of secondary osteons, number of cement line interfaces, and amount of remodeled bone. The densitometry measurements quantified the density of the bone specimens. For both 
measurements, the microradiographs were observed under white light using an Olympus BX41 microscope (Olympus Optical Co., Ltd., Center Valley, PA) and the 10X objective.

\subsection{Histomorphometry}

For these measurements, every cortical beam from each anatomical sector (cranial, caudal, craniolateral, craniomedial, caudomedial, and caudolateral) was divided into four quadrants. To provide better precision, measurements were taken in each quadrant and then the values averaged. The measurements within each quadrant were taken with the assistance of a Merz grid at 100x magnification (Figure 3). To quantify porosity, the thirty six point markers on the Merz grid were used as reference points. Each time one of these point markers fell on a porosity it was counted as one (Figure 15). Porosity was considered a point that did not contain bone tissue and typically occurs from Haversian canals, Volkmann's canals, or remodeling cavities. The points were also used for finding the amount of remodeled bone. Each time one of the points landed on remodeled tissue it was counted as one (Figure 16). Remodeled tissue was identified by either whole or partial secondary osteons; primary osteons were not included in this count. The numbers for the porosity and the amount of bone cannot exceed 36, the number of points on the Merz grid [37]. 


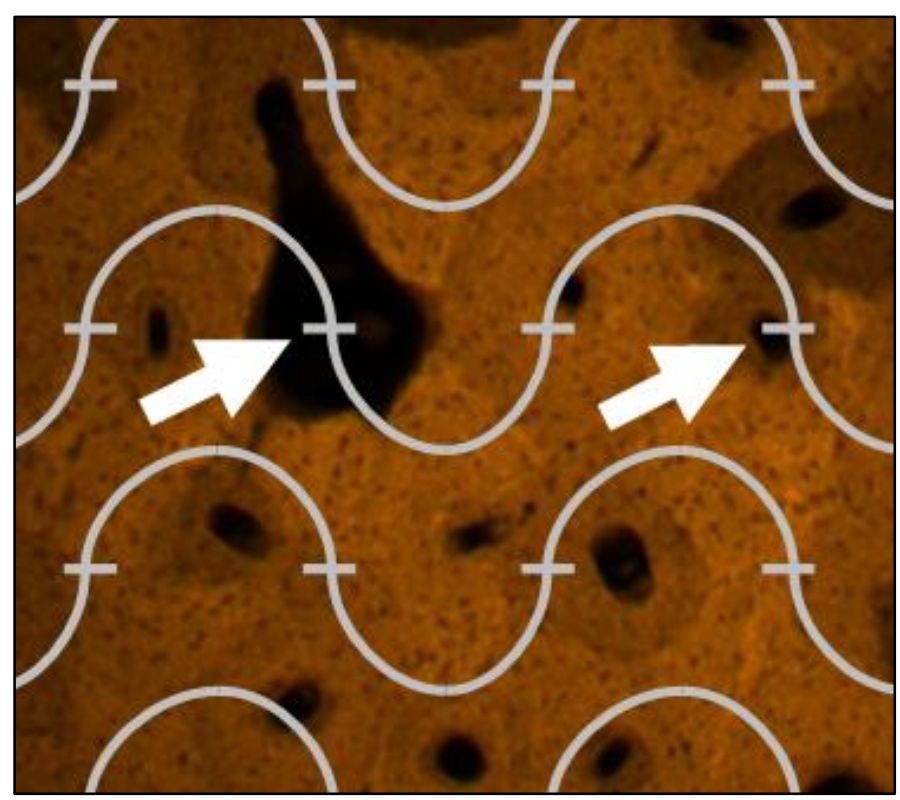

Figure 15 Merz grid point markers located on a porosity

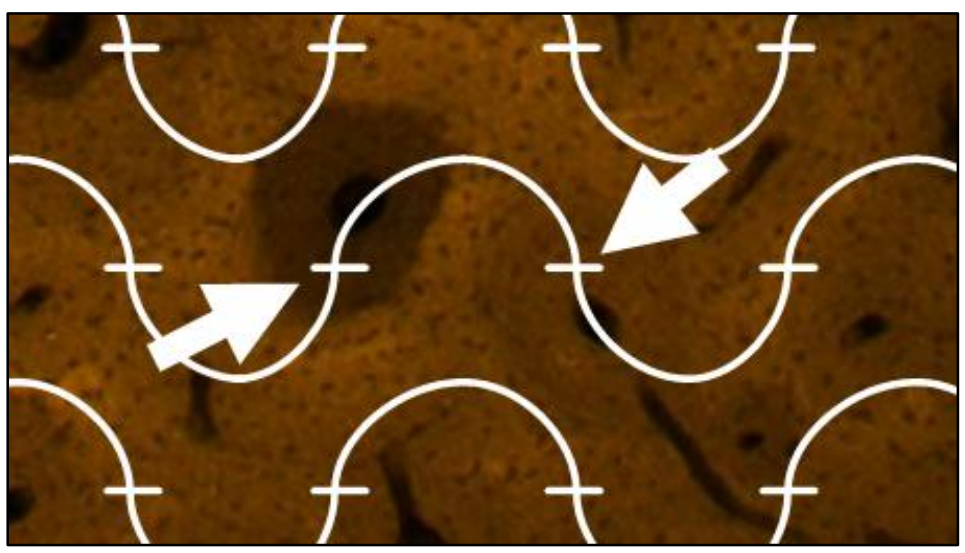

Figure 16 Merz grid points located on remodeled tissue

To quantify the number of secondary osteons, the borders of the Merz grid were used. Each time a secondary osteon was within the grid it was recorded as one (Figure 17). Prominent features of a secondary osteon were used to help identify them, particularly their circular nature, centering on a Haversian canal, circular pattern of osteocyte lacunae, and a cement line. In an effort to minimize the amount of repeating 
counts made in the four quadrants, if a secondary osteon fell on the top or right border of the grid it was counted. However, if the osteon was on the left or bottom border it was not counted.

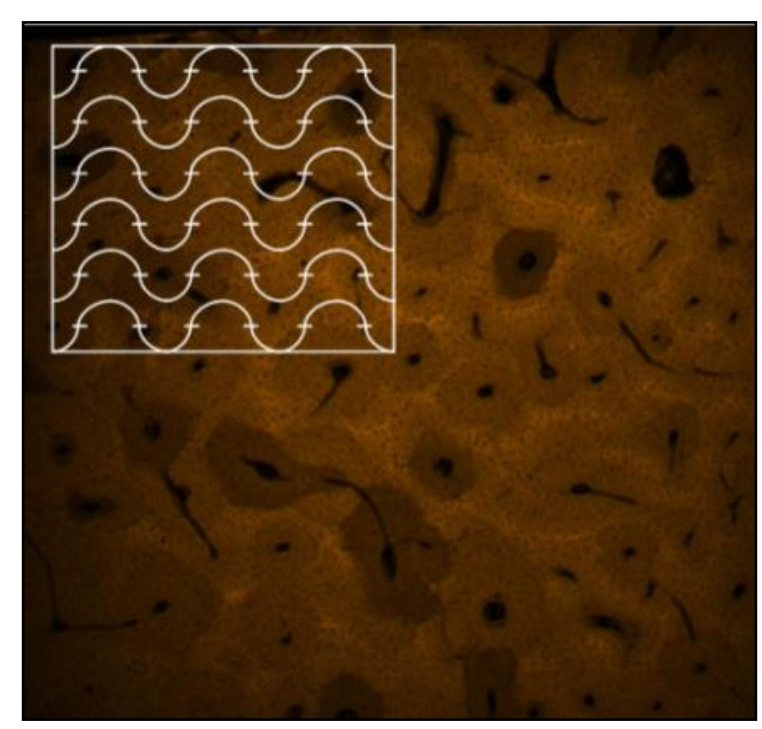

Figure 17 Secondary osteons located within the scope of the Merz grid The six, linked semicircular lines on the Merz grid were guides for determining the number of cement line interfaces. Each time the line entered or left a secondary osteon, crossing the cement line, it was counted as one (Figure 18). After completion of all four measurements, the measurements were then repeated for the other three quadrants of that section. The collected raw data was then used to compute the amount of remodeling that occurred in the bone by finding the ratio of bone volume to tissue volume (BV/TV), percent of remodeled tissue and material, number of cement line interfaces as a function of tissue and material volume, and mean secondary osteonal radius. 


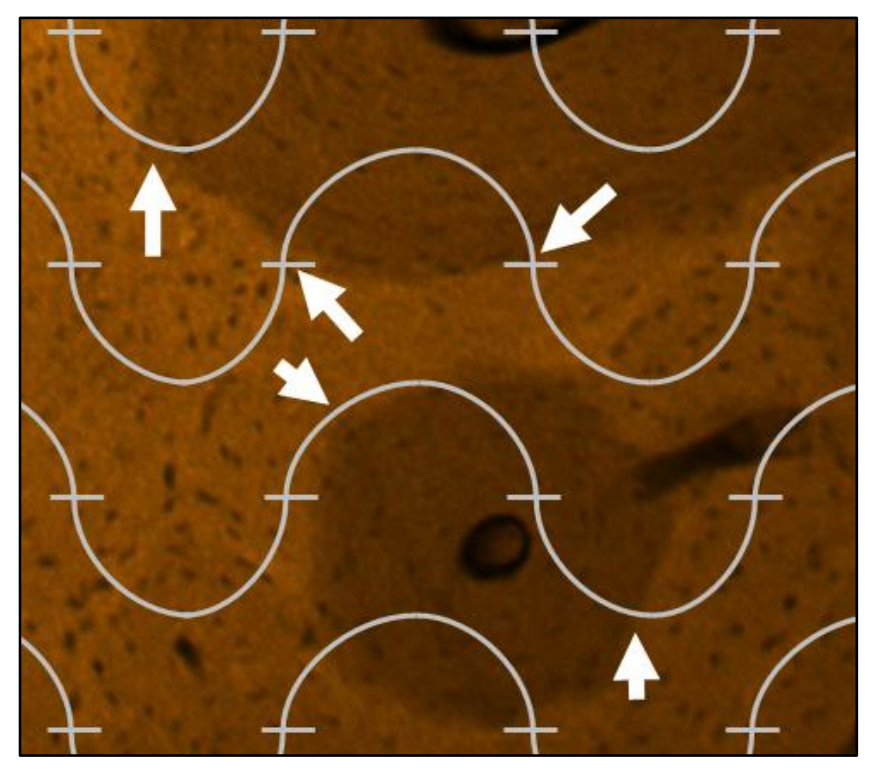

Figure 18 Use of the Merz grid to count the number of cement line interfaces on secondary osteons

$\mathrm{BV} / \mathrm{TV}$ indicates the amount of porosity. It is calculated by dividing the porosity

by 36 , the number of points on the Merz grid, and subtracting that from one (Equation 1).

$$
\frac{B V}{T V}=1-\frac{\text { porosity }}{36}
$$

The amount of remodeling was then quantified in two ways: in the material and tissue. The percent of tissue remodeled is the amount of remodeling that occurs in the entire section. While the percent of material remodeled is the amount of bone that is remodeled and excludes the porosity components. Percent tissue modeled was calculated by dividing the points on remodeled bone by 36 . The percent of remodeled material was calculated by dividing the points on remodeled bone by 36 minus the points that lay on a porosity. The calculations for cement line interface were calculated in a similar manner. The number of tissue cement line interfaces was the raw count of cement line interfaces. The number of material cement line interfaces was the raw count of cement line interfaces divided by the BV/TV. The final calculation was for mean secondary osteon radius. This 
was calculated by multiplying the percent of tissue remodeled by 632025 , a constant determined by Wessel [39], divided by the square root of $\Pi$, times the number of secondary osteons (Equation 2).

Mean Secondary Osteon Radiu $=\frac{\text { Percent of tissue remodeled } \times 632025}{\sqrt{\pi \times \text { number of secondary osteons }}}($ Eq. 2)

\subsection{Densitometry}

In order to quantify the bone density, a reference material of known composition and thickness was used. For this study, aluminum was the implemented material. Aluminum is commonly used in studies involving cortical bone due to its similar x-ray attenuation. The uniformity of aluminum allows for accurate measurements of its thickness $[38,40]$. The measurements of density are described as equivalent thickness of aluminum (ETA) in units of millimeters. The slides were again observed under white light using a BX41 polarizing light microscope. Images were captured of the bone samples and each level of the step wedge, using a Retiga EXi color camera (QImaging, Surrey, BC, Canada) and the Q Capture Pro imaging program (QImaging). To begin, the light intensity was adjusted to allow the best visualization of the structures and a uniform light field within the sample. This light intensity remained the same for pictures of the aluminum step wedge and the sample. Each sample was again divided into four quadrants, with a picture taken of each quadrant at 100x magnification. Only one image was taken for each level of the aluminum step wedge at 40x magnification. In addition to a picture of each step, an image was also taken when the camera was off to control for electrical noise. Each sample was compared to its respective step wedge on the 
microradiograph to determine the density of each sample. The exposure and light settings were reset for each microradiograph.

Image analysis for all the pictures was performed using Image $\mathrm{J}$ (Wayne Rashband (NIH)). The appearances of the image are affected by the density of the material. The thicker and denser materials appear lighter and brighter. As seen in the step wedge, as the thickness of aluminum increases the image brightness also increases (Figure 19)

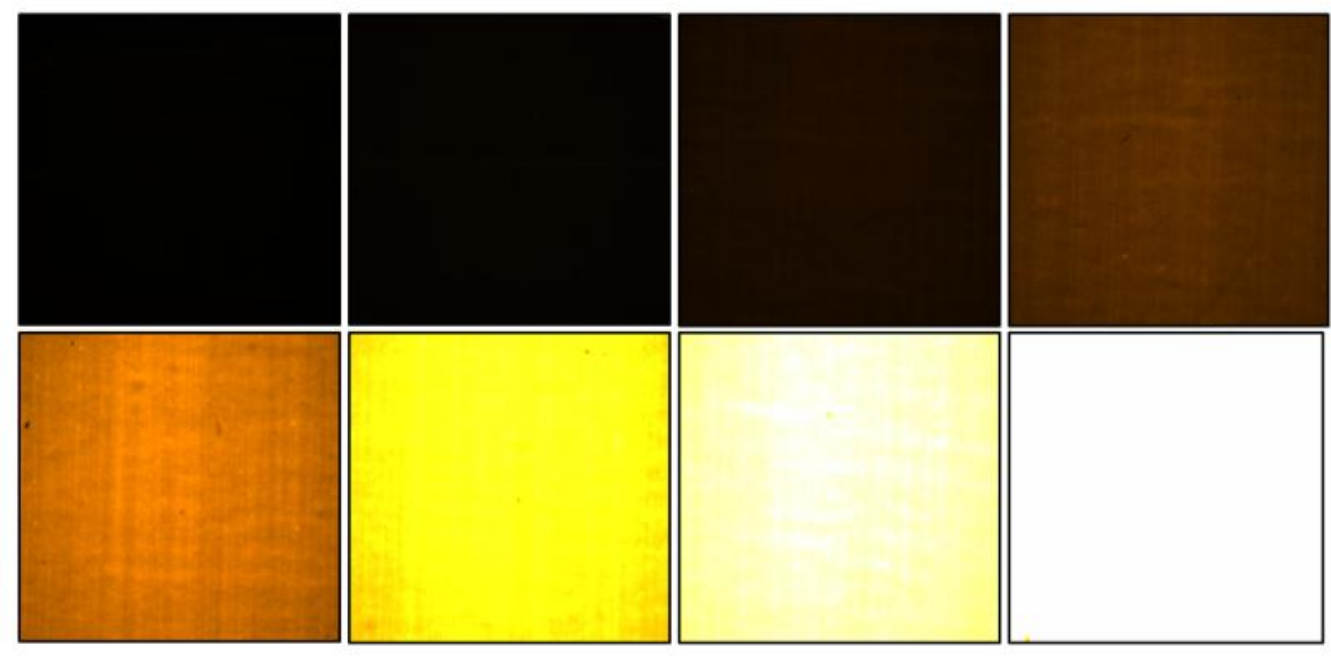

Figure 19 Images of each level of an aluminum step wedge, increasing thickness from left to right, top to bottom $(0 \mathrm{~mm}, 0.02 \mathrm{~mm}, 0.04 \mathrm{~mm}, 0.06 \mathrm{~mm}, 0.08 \mathrm{~mm}, 0.1 \mathrm{~mm}, 0.12 \mathrm{~mm}$, and $0.14 \mathrm{~mm}$ respectively)

Each level in the step wedge is correlated with an increase in thickness of aluminum with each step equaling $0.02 \mathrm{~mm}$. Using the histogram option in Image $\mathrm{J}$, the number of pixels within the image was measured at every pixel intensity from 0 to 255 . From there, mean pixel intensity could be determined for each level in the step wedge and this served as the key. The aluminum thickness was graphed against corresponding mean pixel intensity and then fit to a 4-parameter sigmoid curve (Equation 3) in 
SigmaPlot 11.0 (Systat Software, Inc.). The sigmoidal fit provided the calibration parameters necessary for determining the ETA of each sample.

$$
y=y_{0}+\frac{a}{1+e^{-\left(\frac{x-x_{0}}{b}\right)}}
$$

After the key had been determined, analysis was performed on the samples. Again using Image J, the brightness was measured at each pixel intensity. This was done for all four quadrants of the sample and then using the calibration curve from the key, the pixel intensity values were converted to ETA. This measurement accounted for porosity, by counting all the non-bone points on the four images and dividing it by 144 , the total possible points. This number represented the proportion of pixels that represent nonbone, Q. Then starting with the lowest ETA pixels, data was deleted until the remaining data was 1 minus $\mathrm{Q}$. These measurements were performed for all the samples and its corresponding step wedge.

\subsection{Statistical Analysis}

To analyze the histomorphometric and densitometry measurements, the data were first tested using a 2-way Repeated-Measures ANOVA in SigmaPlot 11.0 (Systat Software, Inc.) to measure the differences in BV/TV, percent of remodeled material, percent of remodeled tissue, CLI (bone), CLI (tissue), and mean secondary osteonal radius. The ANOVA model was performed to compare the effects of season of sacrifice and anatomical sector of the bone on the remodeling parameters. In addition to the main effects, the interaction between season and sector were also evaluated. Comparisons in the analysis were made using post-hoc Fisher Least Significant Difference (LSD) tests. 
The histomorphometric measurements for the control sheep were done as part of a previous thesis and was incorporated into this study to allow for comparison between the control and OVX groups [37]. Thus, a second analysis was performed using a 3 -way ANOVA in SPSS 19.0 to measure the differences in BV/TV, percent of remodeled bone, percent of remodeled tissue, CLI (bone), CLI (tissue), and mean secondary osteonal radius. This model tested the differences in the interaction of season, sector, and treatment across all subjects in addition to the main effects. The comparisons for main effects were again made using a post-hoc Fisher LSD test. The interactive effects were performed using t-tests. In all of the statistical analysis, p-values less than 0.05 were considered significant. 


\section{RESULTS}

\subsection{Histomorphometry}

Measurements were taken for autumn, winter, and spring. The histomorphometric measurements for summer were previously made as part of a larger experiment [41]. For each sector, the average value of the four quadrants was found for BV/TV, the percent of remodeled tissue, the percent of remodeled material, mean secondary osteon radius, cement line interfaces of the tissue, and cement line interfaces of the material. For each season and anatomical sectors, the averages of the remodeling factors and their standard error are found in Tables I, II, III, IV, V, and VI.

Table I Bone volume to tissue volume for adult 12 month OVX ovine compact bone

\begin{tabular}{|l|c|c|}
\hline \multicolumn{3}{|c|}{ BV/TV } \\
\hline \multicolumn{3}{|c|}{ Season } \\
\hline Autumn & Mean & Standard Error \\
\hline Winter & 0.927 & 0.00567 \\
\hline Spring & 0.919 & 0.00567 \\
\hline Summer & 0.930 & 0.00525 \\
\hline \multicolumn{3}{|c|}{ Anatomical Sector } \\
\hline \multicolumn{1}{|c|}{ Group } & Mean & Standard Error \\
\hline Craniomedial & 0.932 & 0.00614 \\
\hline Cranial & 0.939 & 0.00555 \\
\hline Craniolateral & 0.936 & 0.00581 \\
\hline Caudomedial & 0.929 & 0.00669 \\
\hline Caudal & 0.929 & 0.00667 \\
\hline Caudolateral & 0.934 & 0.00704 \\
\hline
\end{tabular}

Table II Percent remodeled tissue for adult 12 month OVX ovine compact bone

\begin{tabular}{|l|c|c|}
\hline \multicolumn{3}{|c|}{ Percent Remodeled Tissue (Remodeled Tissue/Total Area) } \\
\hline \multicolumn{3}{|c|}{ Season } \\
\hline Group & Mean & Standard Error \\
\hline Witumn & 33.7 & 0.0276 \\
\hline Spring & 27.1 & 0.0276 \\
\hline Summer & 20.7 & 0.0255 \\
\hline
\end{tabular}




\begin{tabular}{|l|c|c|}
\hline \multicolumn{3}{|c|}{ Anatomical Sector } \\
\hline \multicolumn{1}{|c|}{ Group } & Mean & Standard Error \\
\hline Craniomedial & 22.6 & 0.0273 \\
\hline Cranial & 30.4 & 0.0242 \\
\hline Craniolateral & 22.9 & 0.0255 \\
\hline Caudomedial & 35.3 & 0.0281 \\
\hline Caudal & 35.3 & 0.0203 \\
\hline Caudolateral & 29.5 & 0.0301 \\
\hline
\end{tabular}

Table III Percent remodeled material for adult 12 month OVX ovine compact bone

\begin{tabular}{|c|c|c|}
\hline \multicolumn{3}{|c|}{ Percent Remodeled Material (Remodeled Tissue/Bone Area) } \\
\hline \multicolumn{3}{|c|}{ Season } \\
\hline Group & Mean & Standard Error \\
\hline Autumn & 36.5 & 0.0287 \\
\hline Winter & 29.6 & 0.0287 \\
\hline Spring & 22.4 & 0.0266 \\
\hline Summer & 33.8 & 0.0315 \\
\hline \multicolumn{3}{|c|}{ Anatomical Sector } \\
\hline Group & Mean & Standard Error \\
\hline Craniomedial & 24.8 & 0.0274 \\
\hline Cranial & 32.3 & 0.0253 \\
\hline Craniolateral & 24.3 & 0.0273 \\
\hline Caudomedial & 32.3 & 0.0307 \\
\hline Caudal & 38.0 & 0.0204 \\
\hline Caudolateral & 31.8 & 0.0335 \\
\hline
\end{tabular}

Table IV Mean secondary osteon radius for adult 12 month OVX ovine compact bone

\begin{tabular}{|l|c|c|}
\hline \multicolumn{3}{|c|}{ Mean Secondary Osteon Radius $(\boldsymbol{\mu m})$} \\
\hline \multicolumn{3}{|c|}{ Season } \\
\hline Group & Mean & Standard Error \\
\hline Wutumn & 58.535 & 3.536 \\
\hline Spring & 57.295 & 3.536 \\
\hline Summer & 56.306 & 3.555 \\
\hline \multicolumn{3}{|c|}{ Anatomical Sector } \\
\hline \multicolumn{1}{|c|}{ Group } & Mean & Standard Error \\
\hline Craniomedial & 55.137 & 2.821 \\
\hline Cranial & 61.650 & 2.744 \\
\hline Craniolateral & 58.527 & 2.741 \\
\hline Caudomedial & 59.559 & 2.741 \\
\hline
\end{tabular}




\begin{tabular}{|l|c|c|}
\hline Caudal & 61.665 & 2.685 \\
\hline Caudolateral & 58.348 & 2.685 \\
\hline
\end{tabular}

Table V Cement line interfaces (tissue) for adult 12 month OVX ovine compact bone

\begin{tabular}{|c|c|c|}
\hline \multicolumn{3}{|c|}{ Cement Line Interfaces (Tissue) $\left(\mathrm{mm} / \mathrm{mm}^{2}\right)$} \\
\hline \multicolumn{3}{|c|}{ Season } \\
\hline Group & Mean & Standard Error \\
\hline Autumn & 23.826 & 2.504 \\
\hline Winter & 23.819 & 2.504 \\
\hline Spring & 21.405 & 2.318 \\
\hline Summer & 32.884 & 2.743 \\
\hline \multicolumn{3}{|c|}{ Anatomical Sector } \\
\hline Group & Mean & Standard Error \\
\hline Craniomedial & 19.654 & 2.35984 \\
\hline Cranial & 28.717 & 2.37181 \\
\hline Craniolateral & 21.542 & 2.10055 \\
\hline Caudomedial & 24.796 & 2.14402 \\
\hline Caudal & 32.046 & 2.00219 \\
\hline Caudolateral & 26.147 & 2.79263 \\
\hline
\end{tabular}

Table VI Cement line interfaces (material) for adult 12 month OVX ovine compact bone

\begin{tabular}{|l|c|c|}
\hline \multicolumn{3}{|c|}{ Cement Line Interfaces (Material) $\left(\mathbf{m m} / \mathbf{m m}^{\mathbf{2}}\right)$} \\
\hline \multicolumn{3}{|c|}{ Season } \\
\hline Group & Mean & Standard Error \\
\hline Autumn & 25.717 & 2.626 \\
\hline Winter & 25.954 & 2.626 \\
\hline Spring & 22.349 & 2.431 \\
\hline Summer & 34.554 & 2.877 \\
\hline \multicolumn{3}{|c|}{ Anatomical Sector } \\
\hline \multicolumn{1}{|c|}{ Group } & Mean & Standard Error \\
\hline Craniomedial & 20.169 & 2.43555 \\
\hline Cranial & 30.035 & 2.51807 \\
\hline Craniolateral & 23.446 & 2.17466 \\
\hline Caudomedial & 26.480 & 2.30447 \\
\hline Caudal & 34.604 & 2.02224 \\
\hline Caudolateral & 28.128 & 2.90092 \\
\hline
\end{tabular}




\subsubsection{Two Way Repeated Measures ANOVA}

A 2-way repeated measures ANOVA was performed to obtain the means and standard errors for the OVX sheep. Additionally, the statistical test was used to determine seasonal and anatomical differences as well as possible seasonality within the anatomical sector. A p-value less than 0.05 was considered significant. Table VII summarizes the pvalues obtained from the 2-way repeated measures ANOVA for each bone remodeling parameter.

Table VII P-values for 2-way repeated measures ANOVA of remodeling parameters

\begin{tabular}{|c|c|c|c|}
\hline \multicolumn{4}{|c|}{ 2-way Repeated Measures ANOVA: p-values } \\
\hline Parameter & Season & Sector & Interaction \\
\hline BV/TV & $\mathbf{0 . 0 0 4}$ & 0.691 & 0.280 \\
\hline Percent Remodeled (Tissue) & $\mathbf{0 . 0 1 1}$ & $\mathbf{0 . 0 0 0}$ & 0.072 \\
\hline Percent Remodeled (Material) & $\mathbf{0 . 0 1 0}$ & $\mathbf{0 . 0 0 0}$ & $\mathbf{0 . 0 4 9}$ \\
\hline Mean Secondary Osteon Radius & 0.443 & 0.564 & 0.711 \\
\hline Cement Line Interface (Tissue) & 0.029 & $\mathbf{0 . 0 0 0}$ & $\mathbf{0 . 0 0 4}$ \\
\hline Cement Line Interface (Material) & 0.031 & $\mathbf{0 . 0 0 0}$ & $\mathbf{0 . 0 0 4}$ \\
\hline
\end{tabular}

A significant difference was seen in the BV/TV between seasons (Figure 20).

Animals sacrificed in summer had significantly larger BV/TV values than all other season. No significant differences were shown among autumn, winter, and spring. There was no significant anatomical variation or seasonality within sector in the BV/TV values. Significant seasonal and anatomical variation was seen in the percent of tissue remodeled as shown in Figure 21 and Figure 22. Higher levels of remodeling were seen on the cranial and caudal regions of the bone. The cranial and caudal regions correspond to areas of high tension and compression respectively [42]. The regions surrounding the neutral axis exhibited lower amounts of remodeled tissue. The p-value for the interaction of season and sector was not significant; however it was near the 0.05 cut off and could potentially provide useful information. There was significant seasonality within region in 
the percent of remodeled material as shown in Figure 23. The caudomedial and craniomedial regions exhibited less variation among the seasons. However, the other sectors showed more variability between seasons and anatomical sector.

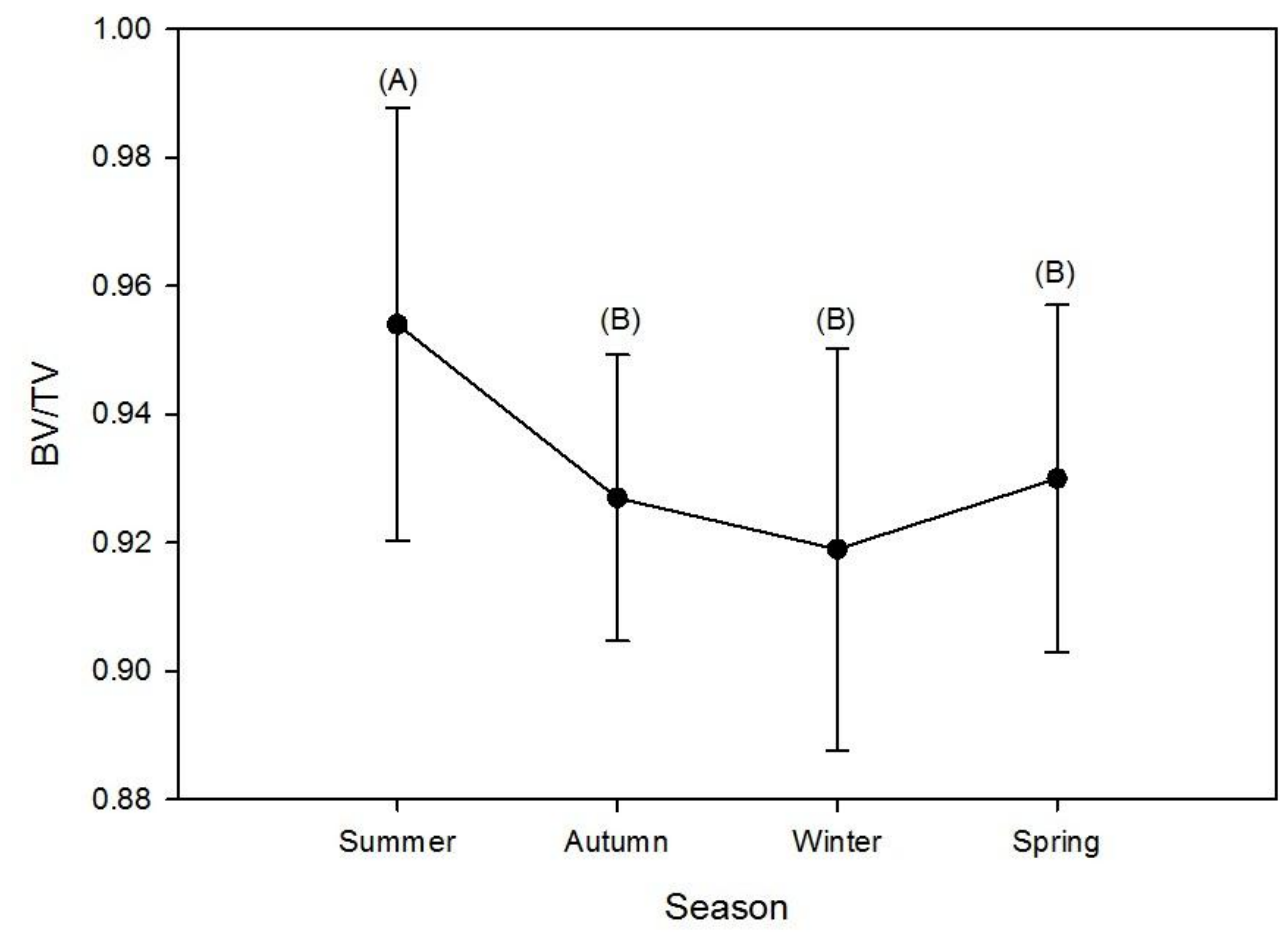

Figure 20 Mean BV/TV values and standard deviations for each season of the OVX sheep 


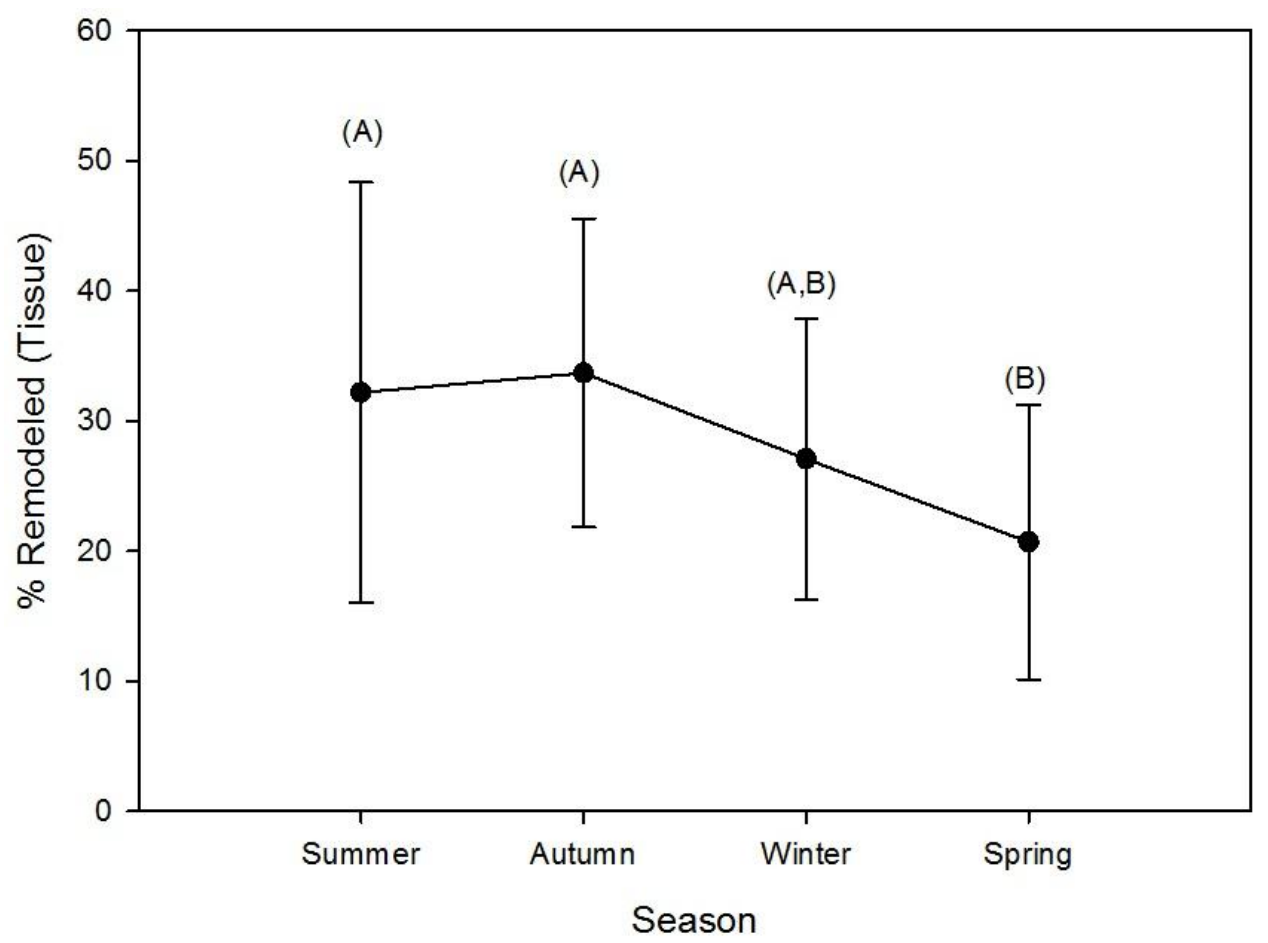

Figure 21 Mean percent of remodeled tissue values and standard deviations for each season of the OVX sheep

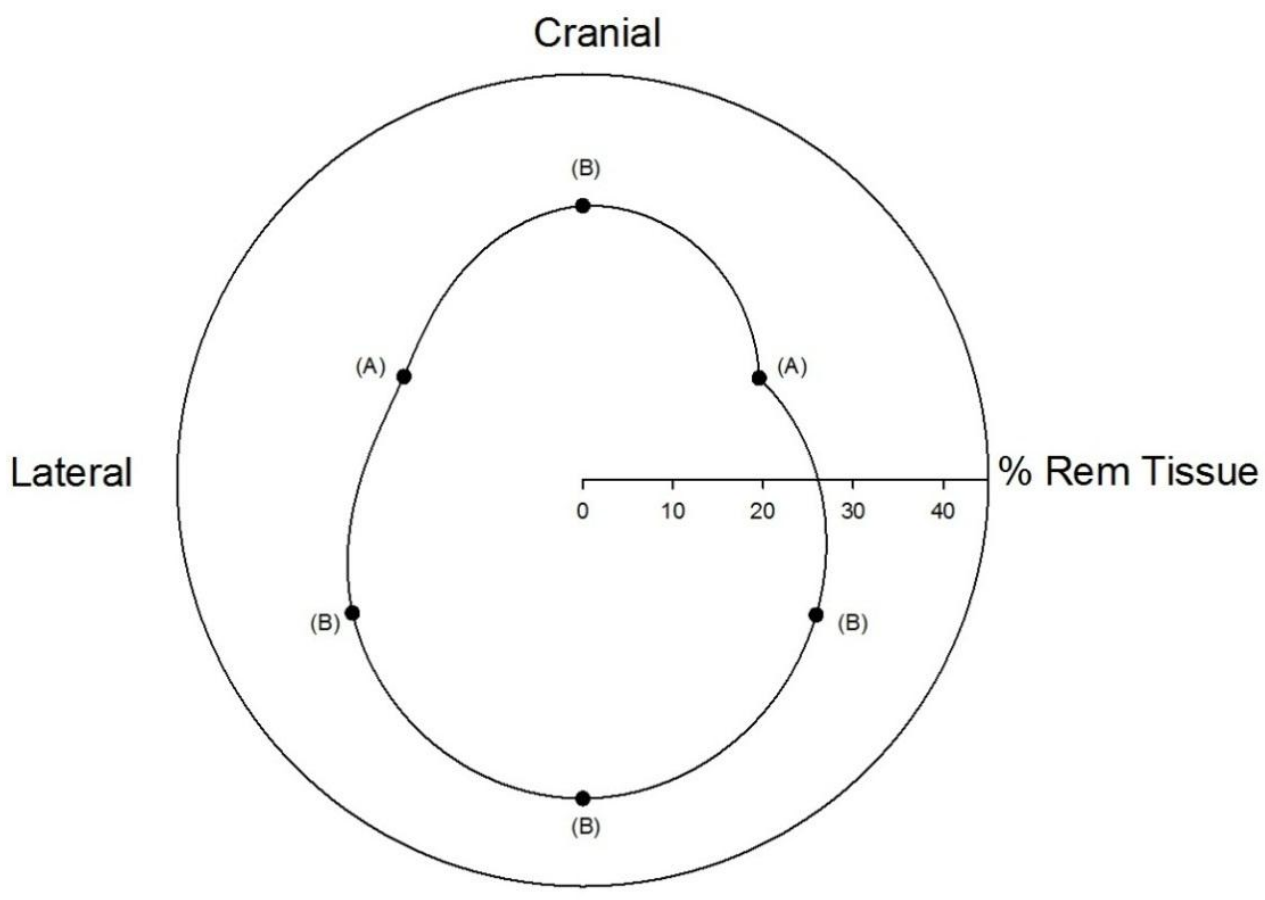

Figure 22 Anatomic variability of the OVX sheep in the percentage of tissue remodeled. The polar graph orients the values for each sector in their approximate anatomical location. The radial distance indicates the value of the remodeling parameter. 


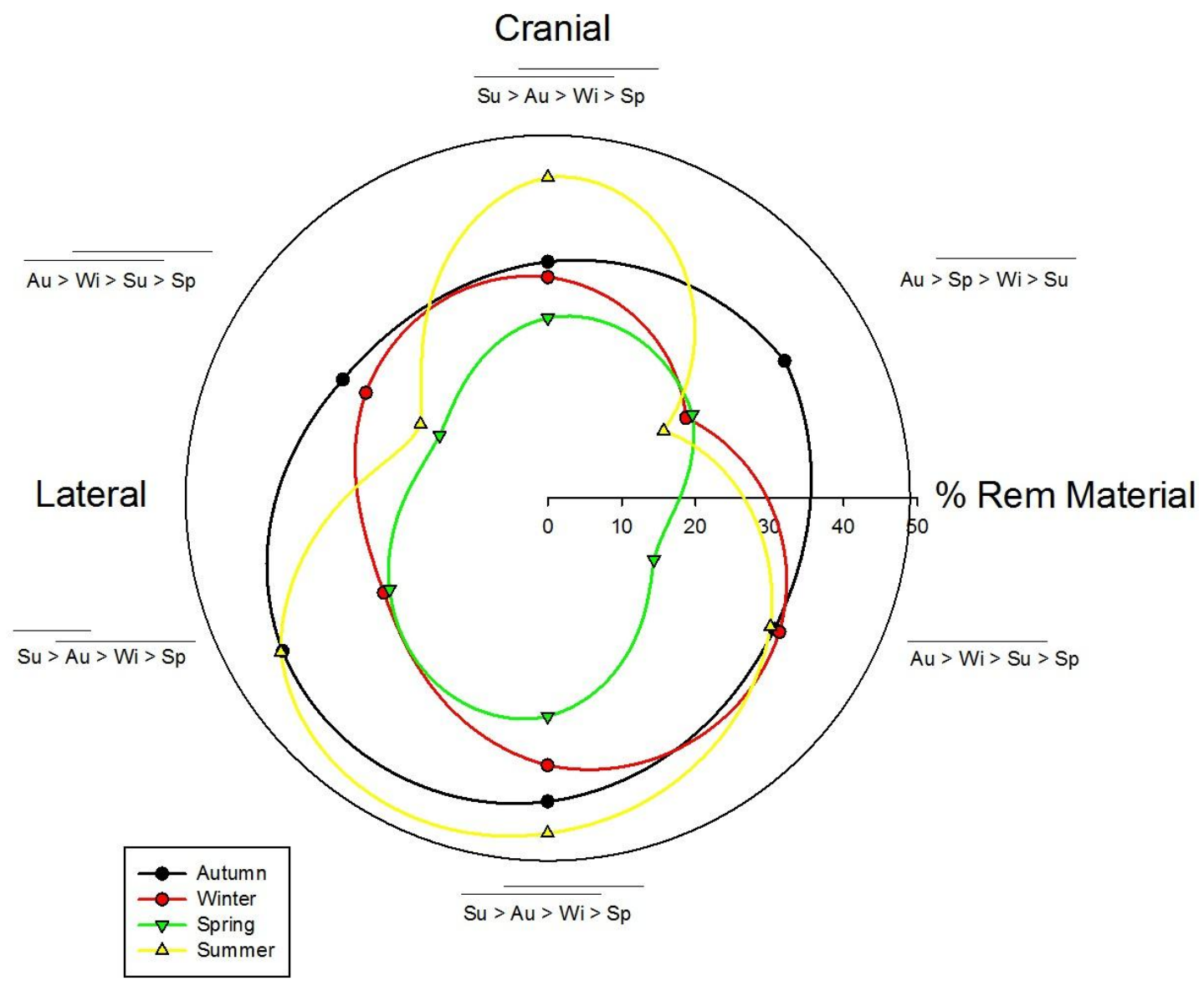

Figure 23 Anatomic variability of OVX sheep within season for the percentage of material remodeled. The polar graph orients the values for each sector in their approximate anatomical location. The radial distance indicates the value of the remodeling parameter.

Analysis of secondary mean osteon radius showed no significant effects of seasonality or anatomical region. Cement line interfaces for both the tissue and material demonstrated significant seasonality within anatomical sectors as seen in Figure 24 and Figure 25. The craniomedial region showed no seasonal variability in the tissue cement line interfaces, as opposed to the other regions which saw the effects of season and anatomical region. Analysis of the material cement line interfaces indicated the craniomedial and craniolateral regions had minimal seasonal effects. However, seasonal and anatomical variation were seen in the other sectors. 


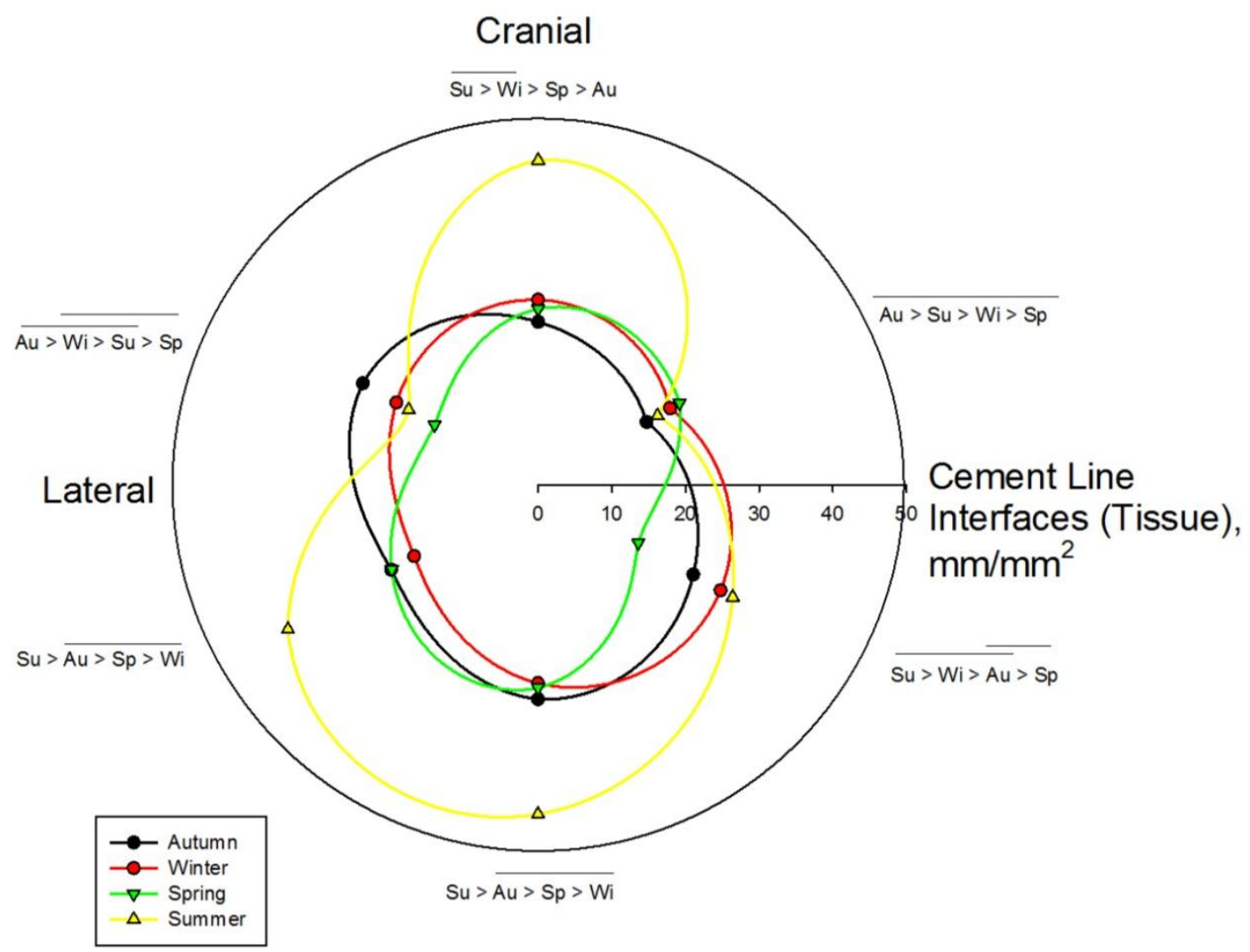

Figure 24 Anatomic variability of OVX sheep within season for the number of tissue cement line interfaces. The polar graph orients the values for each sector in their approximate anatomical location. The radial distance indicates the value of the remodeling parameter. 


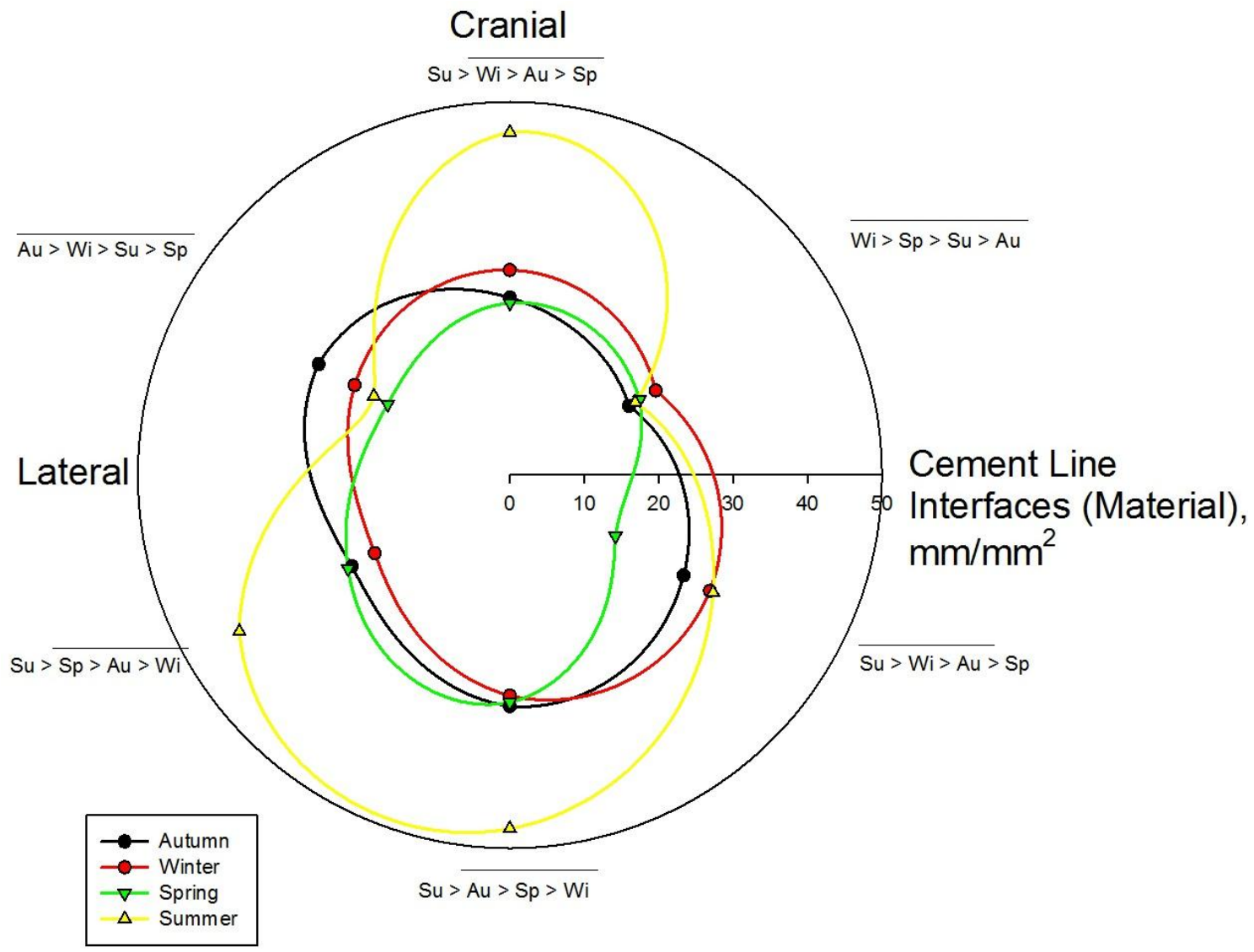

Figure 25 Anatomic variability of OVX sheep within season for the number of material cement line interfaces. The polar graph orients the values for each sector in their approximate anatomical location. The radial distance indicates the value of the remodeling parameter.

\subsubsection{Three Way ANOVA}

The histomorphometric data for the 12 month control sheep was collected as part of a previous thesis [37]. A 3-way ANOVA was performed to obtain the means and standard errors for the control and OVX data. Additionally, the statistical test was used to assess seasonal, anatomical, and treatment differences as well as possible interactions between all three factors. A p-value less than 0.05 was considered significant. Table VIII summarizes the p-values obtained from the 3-way ANOVA for each parameter. 
Table VIII P-values for 3-way ANOVA for comparison of OVX and control remodeling parameters

\begin{tabular}{|c|c|c|c|c|c|c|c|}
\hline \multicolumn{2}{|c|}{ 3-way ANOVA: p-values } \\
\hline Parameter & Season & Sector & Treatment & $\begin{array}{c}\text { Season } \\
\text { \& Sector }\end{array}$ & $\begin{array}{c}\text { Season \& } \\
\text { Treatment }\end{array}$ & $\begin{array}{c}\text { Sector \& } \\
\text { Treatment }\end{array}$ & $\begin{array}{c}\text { Season, } \\
\text { Sector, \& } \\
\text { Treatment }\end{array}$ \\
\hline BV/TV & $\mathbf{0 . 0 0 0}$ & $\mathbf{0 . 0 4 1}$ & 0.060 & 0.052 & 0.093 & 0.121 & $\mathbf{0 . 0 1 8}$ \\
\hline $\begin{array}{c}\text { Percent } \\
\text { Remodeled } \\
\text { (Tissue) }\end{array}$ & $\mathbf{0 . 0 0 3}$ & $\mathbf{0 . 0 0 0}$ & 0.455 & 0.872 & 0.219 & 0.430 & $\mathbf{0 . 0 1 2}$ \\
\hline $\begin{array}{c}\text { Percent } \\
\text { Remodeled } \\
\text { (Material) }\end{array}$ & $\mathbf{0 . 0 0 4}$ & $\mathbf{0 . 0 0 0}$ & 0.796 & 0.803 & 0.171 & 0.428 & $\mathbf{0 . 0 0 8}$ \\
\hline $\begin{array}{c}\text { Mean } \\
\text { Secondary } \\
\text { Osteon } \\
\text { Radius }\end{array}$ & $\mathbf{0 . 0 0 0}$ & 0.602 & 0.084 & 0.544 & 0.844 & 0.816 & 0.914 \\
\hline $\begin{array}{c}\text { Cement } \\
\text { Line } \\
\text { Interface } \\
\text { (Tissue) }\end{array}$ & $\mathbf{0 . 0 1 0}$ & $\mathbf{0 . 0 0 0}$ & $\mathbf{0 . 0 0 3}$ & 0.358 & 0.116 & 0.089 & $\mathbf{0 . 0 0 0}$ \\
\hline $\begin{array}{c}\text { Cement } \\
\text { Line } \\
\text { Interface } \\
\text { Material) }\end{array}$ & $\mathbf{0 . 0 1 3}$ & $\mathbf{0 . 0 0 0}$ & $\mathbf{0 . 0 0 7}$ & 0.256 & 0.146 & 0.095 & $\mathbf{0 . 0 0 0}$ \\
\hline
\end{tabular}

Seasonality and anatomical sector within treatment had a significant effect on the BV/TV as seen in Figure 26. The control sheep have higher ratios of BV/TV when compared to the OVX sheep across all seasons. However, the differences are only statistically different during the autumn, winter, and spring seasons. The percent of remodeled tissue and bone showed significant differences due to season (Figure 27 and Figure 29) and anatomical variation (Figure 28 and Figure 30). In the percent of remodeled tissue, there was a significant differnce between the control and OVX sheep during spring. In addition, there was also a difference related to anatomical region. In the cranial and caudolateral regions, the level of tissue remodeling of was significantly different than the control with remodeling decreasing with OVX. The control sheep also 
exhibited significantly larger amounts of material modeling during spring when compared to the OVX sheep. An overall effect of sector was seen in the data for percent of remodeled material, however there were no significant differences between the control and OVX sheep. There was a significant difference in the interaction of season, treatment, and sector in the percent of material and tissue remodeled.

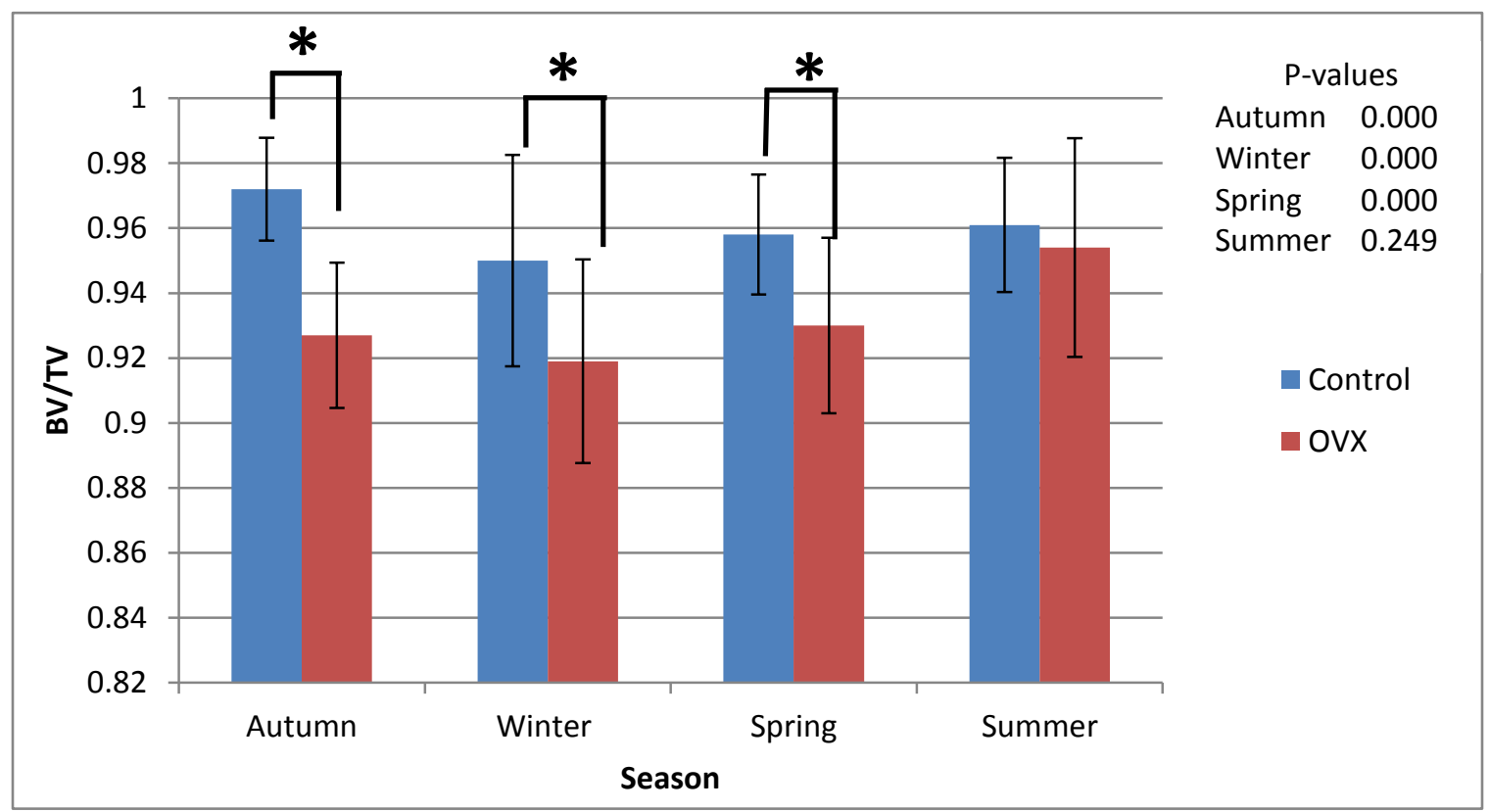

Figure 26 Mean BV/TV and standard deviations for control and OVX sheep for each season. 


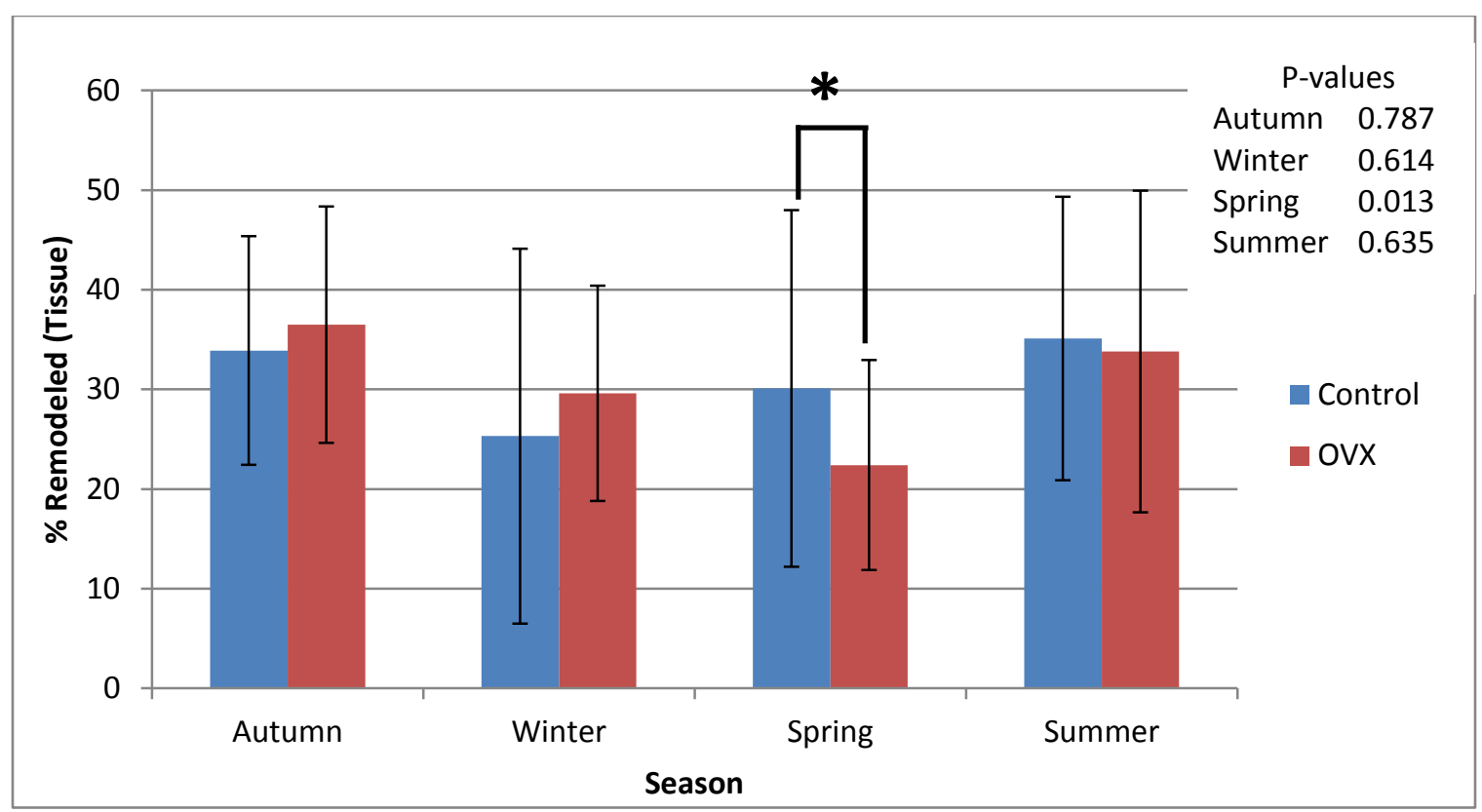

Figure 27 Mean percentage of tissue remodeled and standard deviations for control and OVX sheep for each season. 


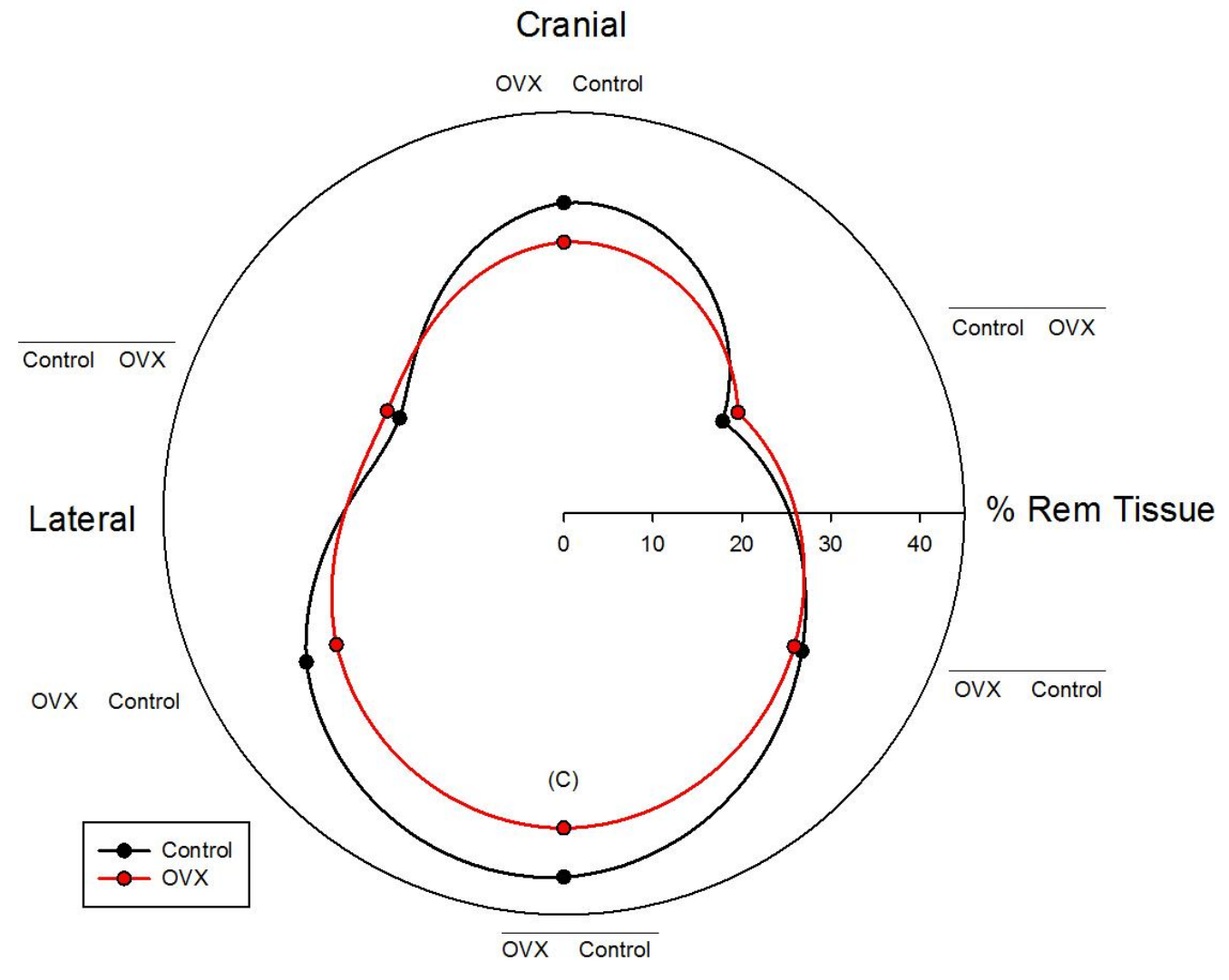

Figure 28 Anatomic variability in the percentage of tissue remodeled for control and OVX sheep. The polar graph orients the values for each sector in their approximate anatomical location. The radial distance indicates the value of the remodeling parameter.

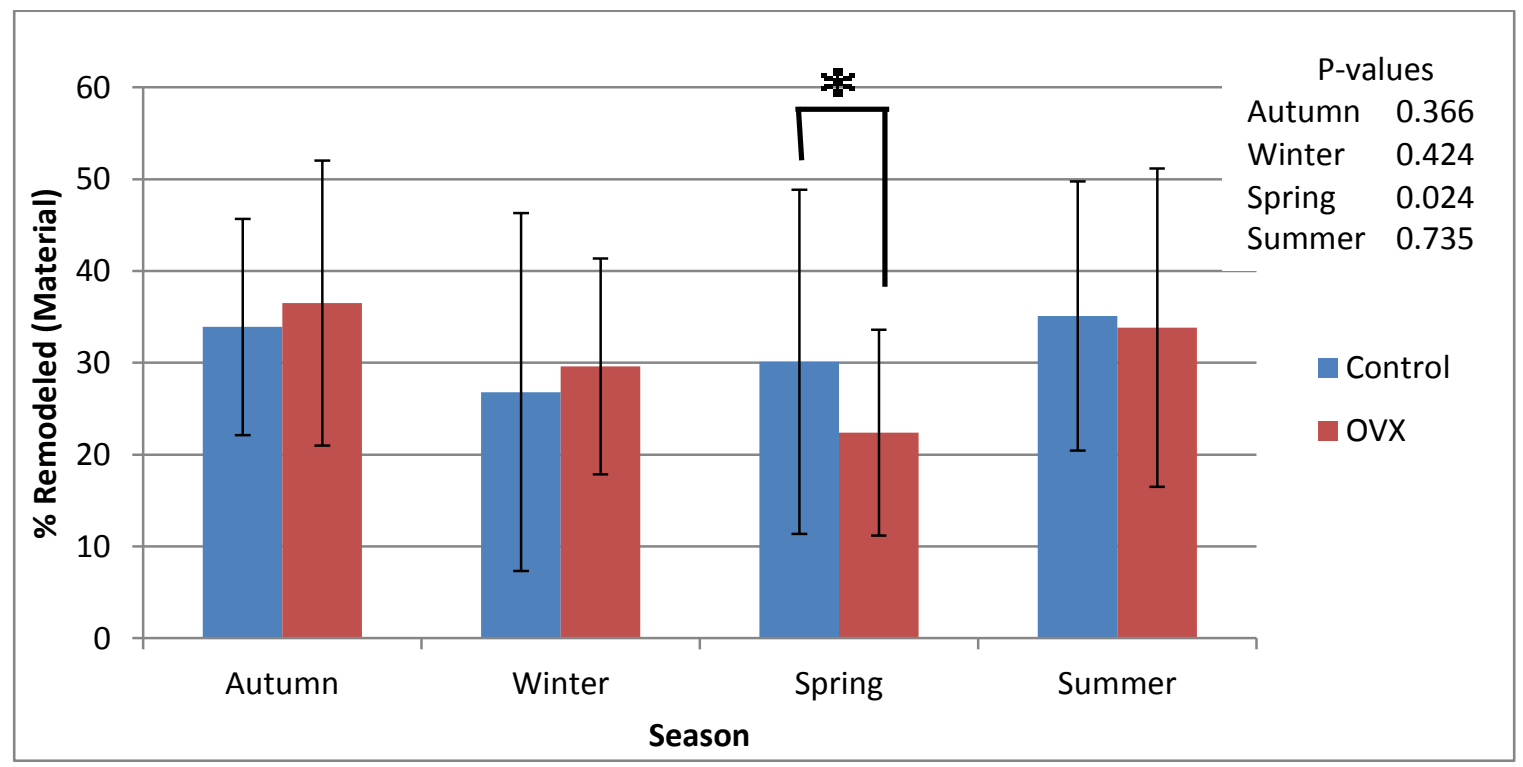

Figure 29 Mean percentage of remodeled material and standard deviations for control and OVX sheep for each season. 


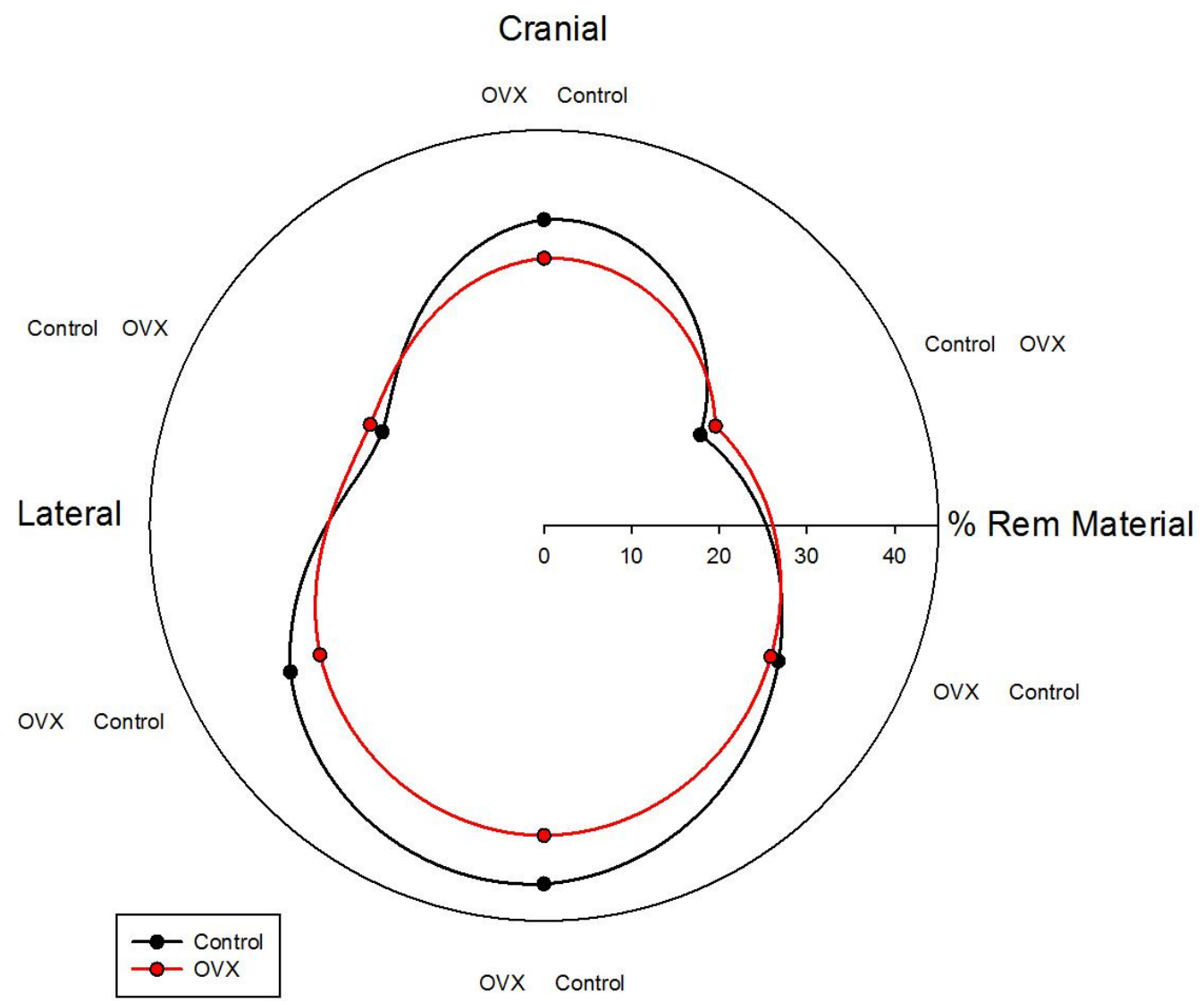

Figure 30 Anatomic variability in the percentage of material remodeled for control and OVX sheep. The polar graph orients the values for each sector in their approximate anatomical location. The radial distance indicates the value of the remodeling parameter.

Statistical analysis of mean secondary osteon values indicates a significant difference due to season (Figure 31). Across the OVX and control sheep, animals sacrificed in summer have larger osteons than all other seasons. However, there was only a significant difference in osteon size in the winter seasons, with the OVX sheep having larger osteons. Additionally, no significant anatomical or treatment differences were seen in the values for mean secondary osteon radius. Cement line interfaces for tissue and bone material showed significant effects from a combination of season, anatomical region, and treatment. For both cement line interface measurements, significant differences between the control and OVX were seen in the autumn and spring sheep 
(Figure 32, Figure 33). However, no significant differences were seen in the winter and summer sheep.

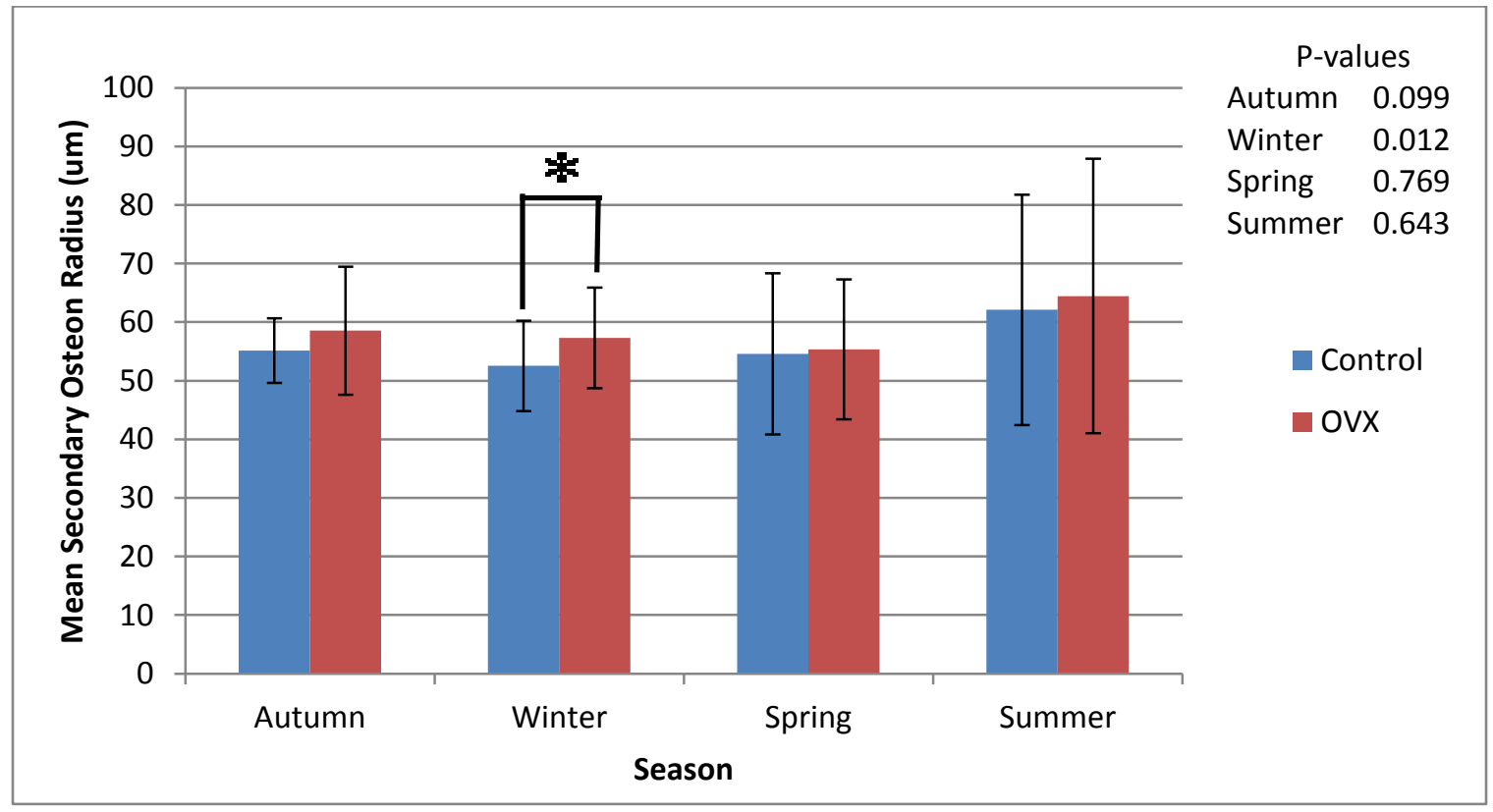

Figure 31 Mean secondary osteon radius (um) and standard deviations for control and OVX sheep for each season.

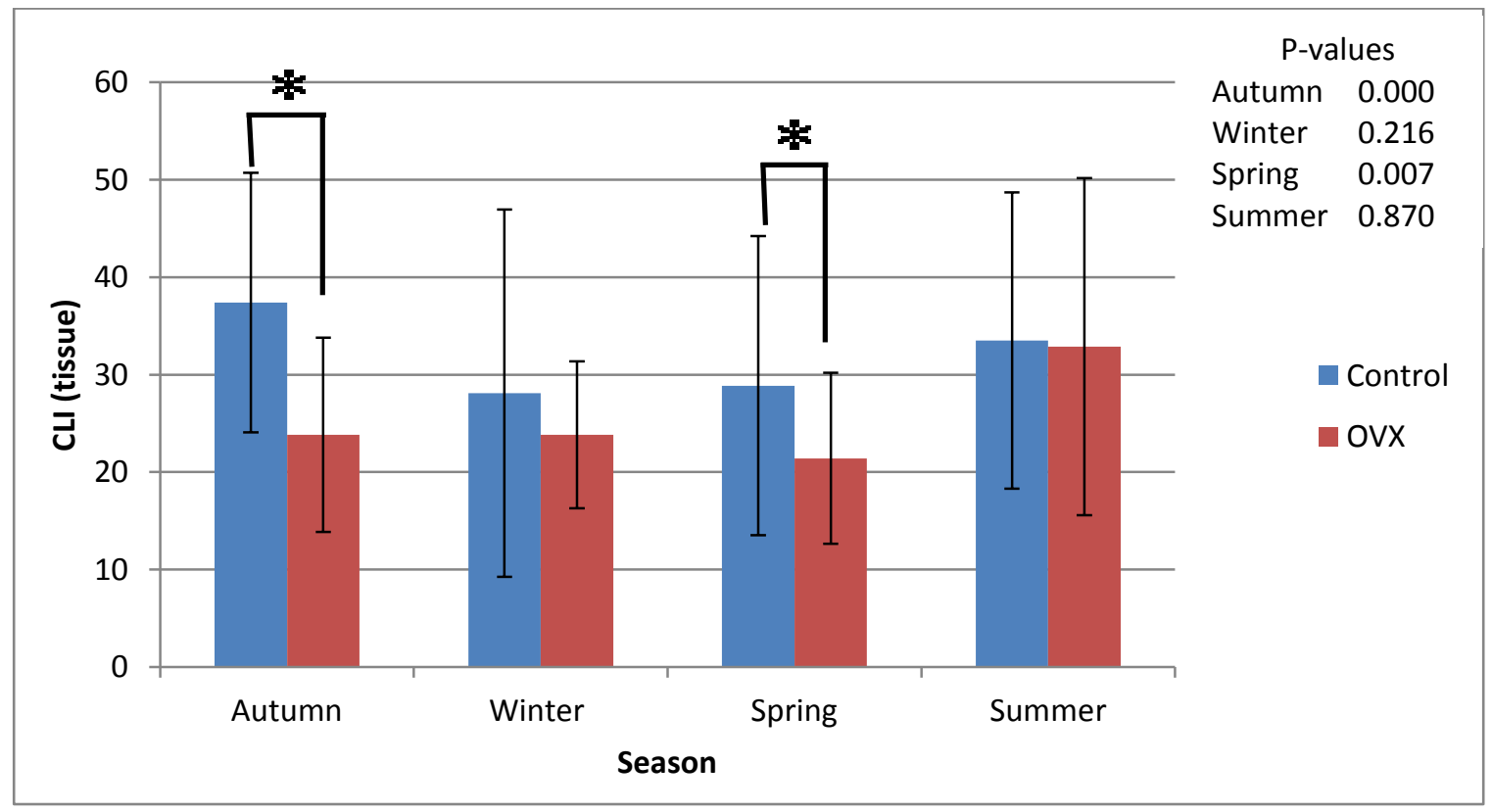

Figure 32 Mean number of tissue cement line interfaces and standard deviations for control and OVX sheep for each season. 


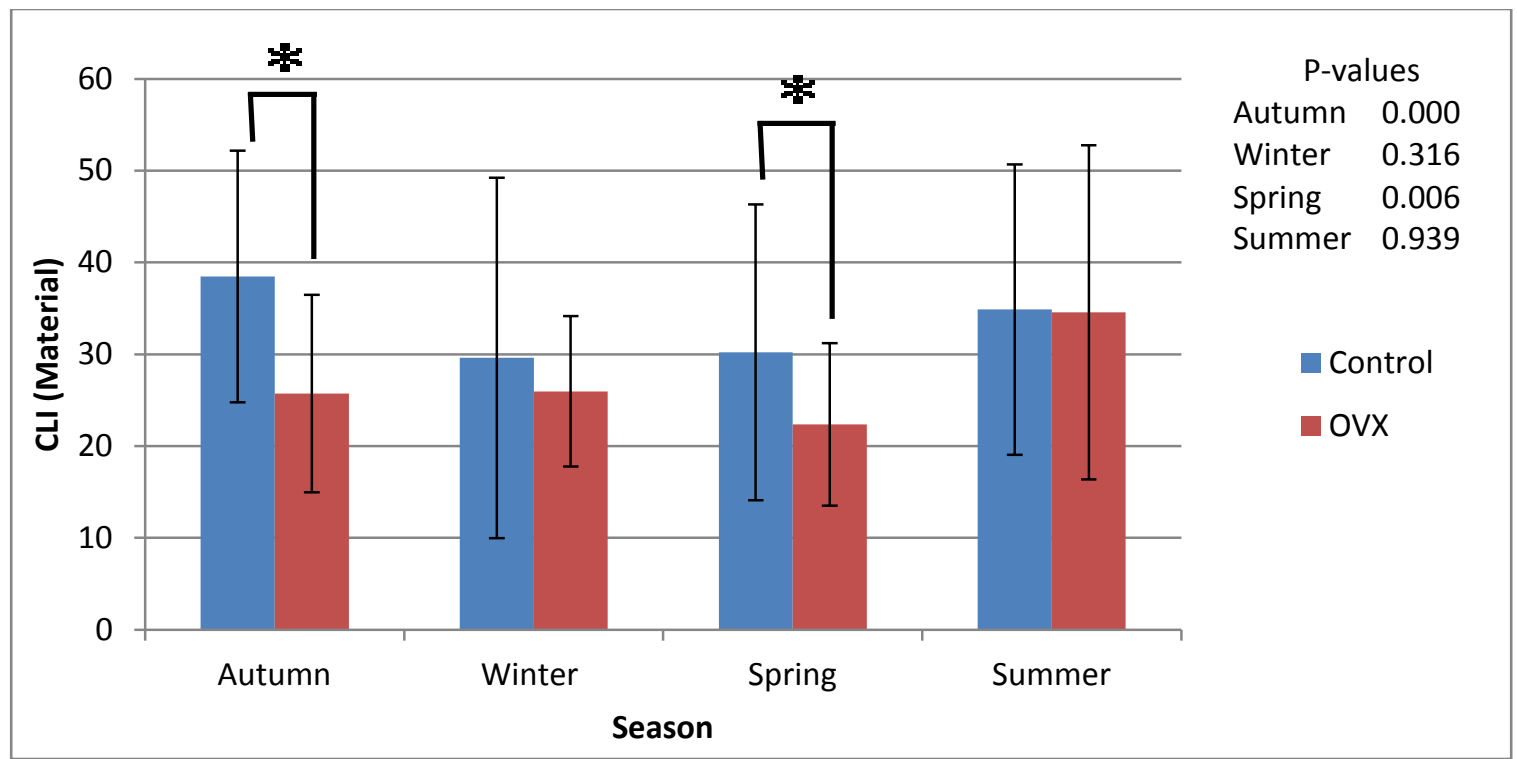

Figure 33 Mean number of material cement line interfaces and standard deviations for control and OVX sheep for each season.

\subsection{Densitometry}

Images were captured of the bone specimens and its corresponding aluminum step wedge. The mean pixel intensity values of the step wedge were determined and in conjunction with its equivalent thickness of aluminum (ETA) were graphed and then fit to a 4-parameter sigmoid curve to establish the calibration curve. The mean pixel intensities were determined for each quadrant of the specimen and using the key the ETA for the specimens could be determined. Measurements were taken for autumn, winter, spring, and summer. The mean ETA values and the standard error for each season and anatomical sector are shown in Table IX. 
Table IX Densitometry values (ETA) for OVX sheep

\begin{tabular}{|c|c|c|}
\hline \multicolumn{3}{|c|}{ Densitometry (ETA) (mm) } \\
\hline \multicolumn{3}{|c|}{ Season } \\
\hline Group & Mean & Standard Error \\
\hline Autumn & 0.0663 & 0.00510 \\
\hline Winter & 0.0608 & 0.00491 \\
\hline Spring & 0.0654 & 0.00446 \\
\hline Summer & 0.0561 & 0.00531 \\
\hline \multicolumn{3}{|c|}{ Anatomical Sector } \\
\hline Group & Mean & Standard Error \\
\hline Craniomedial & 0.0650 & 0.00159 \\
\hline Cranial & 0.0597 & 0.00159 \\
\hline Craniolateral & 0.0643 & 0.0174 \\
\hline Caudomedial & 0.0627 & 0.0164 \\
\hline Caudal & 0.0577 & 0.0171 \\
\hline Caudolateral & 0.0633 & 0.00164 \\
\hline
\end{tabular}

Statistical analysis indicates a significant anatomical difference in the density of the bone (Figure 34). The densest part of the bone was observed in the craniomedial region. Higher levels of density were also seen in the regions surrounding the neutral axis, the craniomedial, craniolateral, caudomedial, and caudolateral regions. The lowest density was seen in the caudal region of the radius/ulna. There was no significant seasonality or seasonality within the anatomical region. 


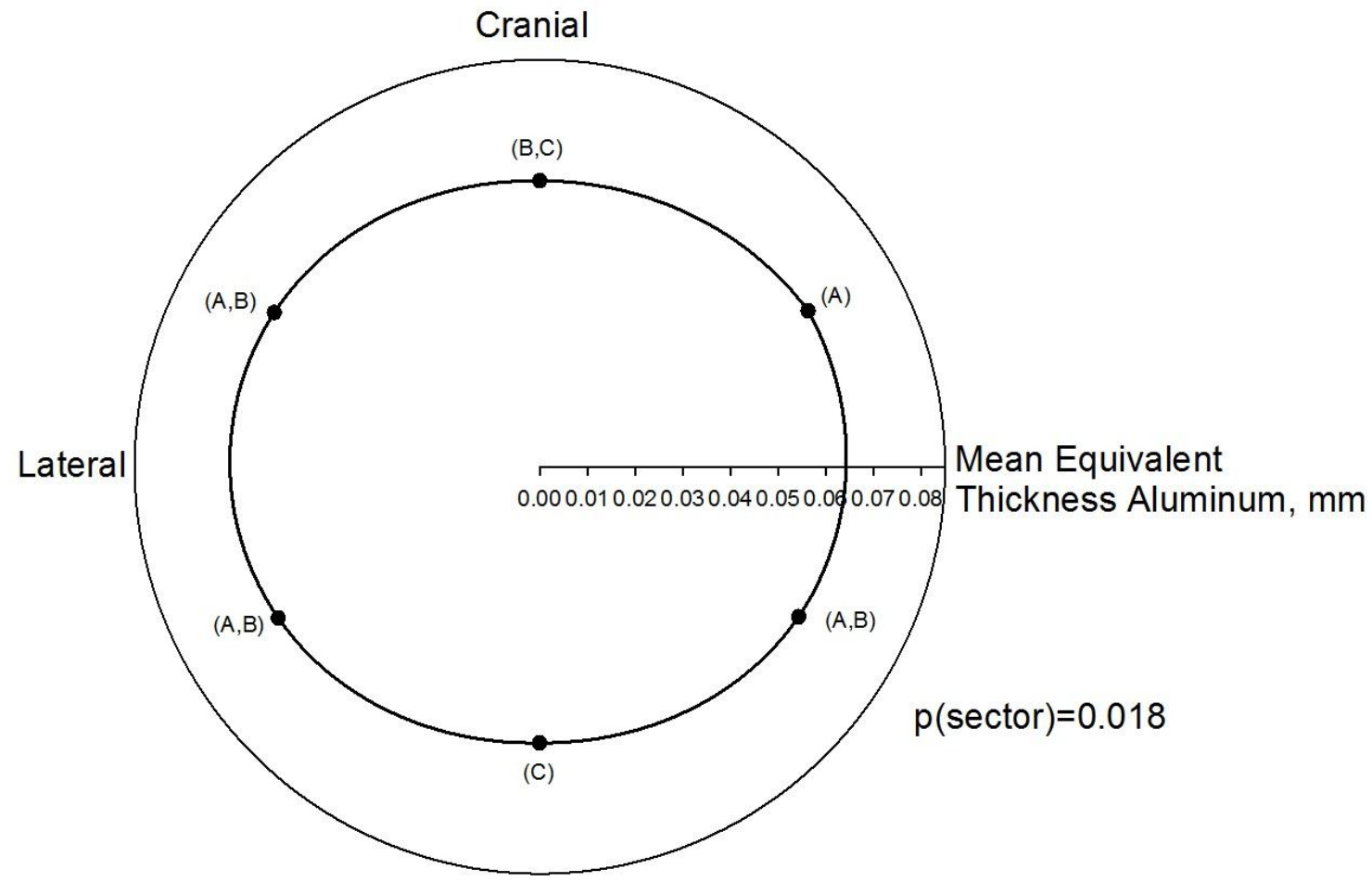

Figure 34 Anatomic variability in density measured in equivalent thickness of aluminum (ETA) for OVX sheep. The polar graph orients the values for each sector in their approximate anatomical location. The radial distance indicates the value of the remodeling parameter. 


\section{DISCUSSION}

Bone disease, specifically osteoporosis, is a health crisis affecting millions of men and women [43]. Osteoporosis is characterized by a decrease in bone mass, making bone more susceptible to fracture. Fragility fractures are the first clinical manifestation of osteoporosis and can occur after minor trauma. Osteoporotic fractures are considered to have the most severe impact on patient's lives, indicated by a decrease in their quality of life and increased dependence on walking aids or help from others [44]. Thus, there is an ever growing need for detection methods for osteoporosis, especially for patients over 50 who suffer fractures. Current detection methods involve dual X-ray absorptiometry to assess patient's bone mineral density. Many patients aren't diagnosed with osteoporosis until after their first fracture and as a result the main challenge in detection methods is identifying patients with fractures who require screening for osteoporosis [45]. Therefore, it is important to understand the risk factors associated with osteoporosis. Increased risk for osteoporosis can be caused by smoking, excessive alcohol and caffeine consumption, increased age and a sedentary lifestyle [43]. Smoking, excessive alcohol and caffeine consumption, and increased age also have shown to increase the risk of osteoporosis related fractures.

There is a great importance in detection methods of osteoporosis, and in addition to dual X-ray absorptiometry other methods have been used for the assessment of bone microarchitecture. Dual X-ray absorptiometry is a well-established method for determining bone mineral density, however that is not the only indicator of bone strength. The fragility of bone is also influenced by mineralization, bone turnover rate, and trabecular and cortical microarchitecture [46]. Computed tomography and magnetic 
resonance are attractive options for assessment of bone microarchitecture. They are feasible primarily because they can be used in vivo. High-resolution peripheral quantitative computed tomography is another promising method to assess bone density and microarchitecture. As seen in Figure 35 it is a method that would allow physicians to determine a patient's osteoporotic risk.

Ovariectomy surgery involves the removal of the ovaries, and as a result can simulate osteopenia in animals. This has been shown to be an effective treatment as many animal models have shown to exhibit osteoporotic characteristics such as increased bone turnover that can eventually lead to bone loss [27]. In addition to providing a model for osteoporosis, ovariectomy provides a means to induce an animal model for postmenopausal osteoporosis. With ovariectomy, the rat model can produce alterations in cancellous bone that is identical to the bone loss seen in the human skeleton during aging and menopause [47]. In addition to rats, monkeys undergo a reduction in cancellous bone in response to ovariectomy. However, due to their high cost they are primarily used after rats and larger animals have been used. 


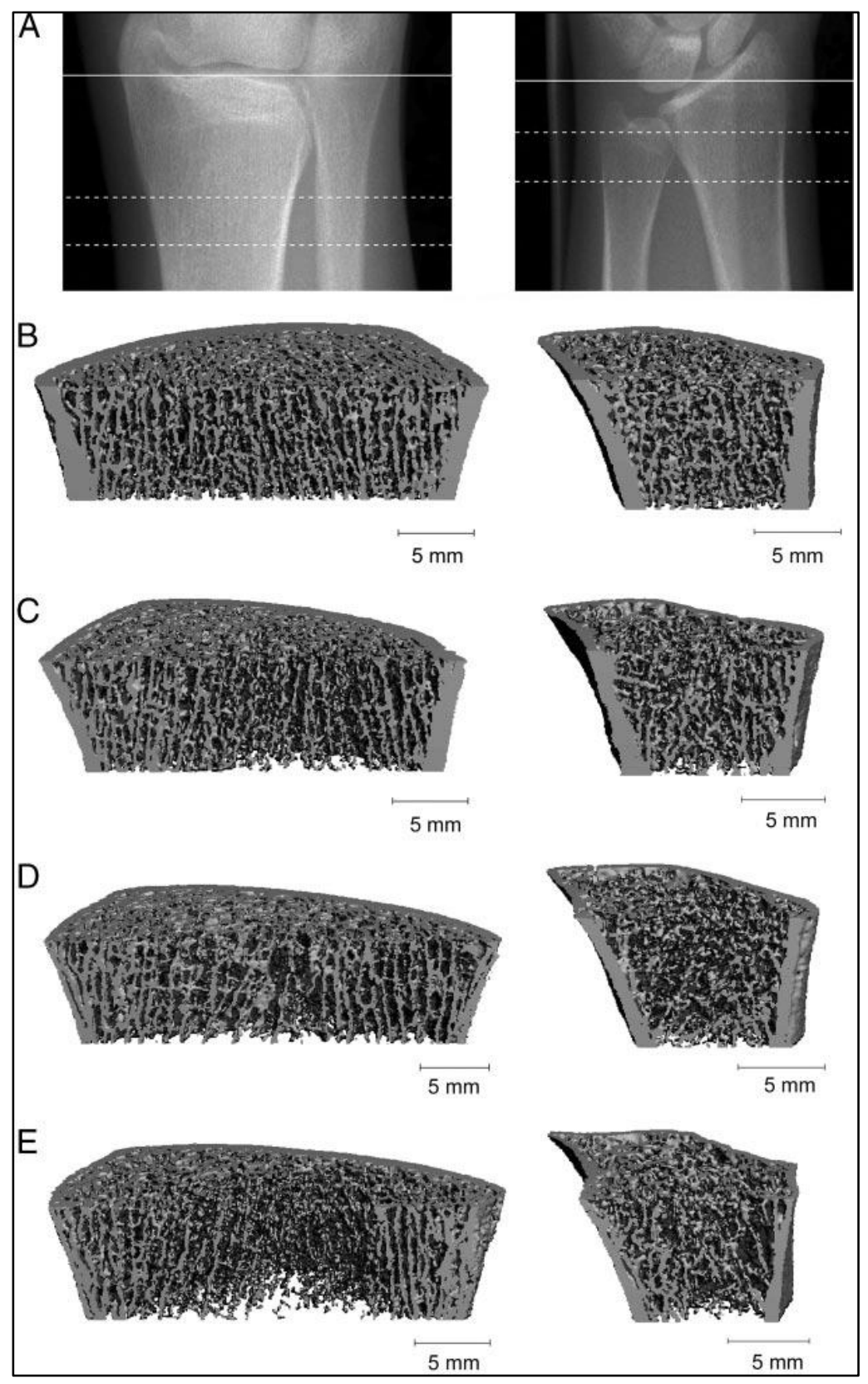

Figure 35 Images of the tibia (left) and radius (right). A. View demonstrating the reference line (solid line) and the measurement site (between dotted lines); B-E, images from a premenopausal (B), postmenopausal osteopenic (C), postmenopausal osteoporotic (D), and postmenopausal severe osteoporotic (E) subject. [48]

Generally, smaller animals such as rats and mice are the initial animal model for testing the pathophysiology of bone loss and subsequent therapy options. These animals are advantageous due to their lower housing costs; however they are not able to mimic 
human osteoporosis as well due to differences in their reproductive physiology, hormone profiles, and biomechanical bone characteristics. Rats and mice also do not provide a large enough model to test full scale device interactions with bone tissue [49]. Primates demonstrate many advantages over other models for osteoporosis due to their resemblance to the human systems. However, as previously stated, primates are extremely costly and difficult to handle and therefore are not the primary model for osteoporosis [27].

There is a need for developing a physiologically relevant and economical large animal model for testing of therapeutic agents to treat osteoporosis. Adult sheep are well suited for this role for many reasons. Sheep present bone loss with estrogen deficiency which is beneficial for analysis of postmenopausal osteoporosis. They exhibit similar hormone profiles to women and Haversian remodeling. Some breeds are seasonally polyestrous and during anestrus periods the animal undergoes a period of estrogen depletion [29]. This contributes to the seasonal effect on the bone remodeling of the animal. As a result, this is an important component that must be considered when implementing sheep as a model for osteoporosis. This is specifically important when comparing different treatments and follow up times. The differences in daylight observed across the seasons plays a crucial part in the activity of the sheep, in turn affecting the animal's loading pattern. The change in loading patterns could potentially affect the rate of bone turnover seen in the varying seasons [33].

The cyclic reproductive pattern seen in sheep is believed to be regulated by melatonin secretion. Research has shown that sheep experience regular 16 to 18 day estrus cycles, period where estrogen has its greatest influence, from mid-November to 
late January. Then from May to September no cycling occurs and sheep go through anestrus cycles, period where the sexual cycle rests $[50,51]$. However, there is disagreement on the exact time periods for the beginning of the anestrus cycle. Other research from Dr. Dana Ruehlman indicates sheep undergo estrous during early summer to late fall followed by the anestrus period in the late fall through the winter [52]. The cortical bone remodeling of the control sheep are directly affected by the hormonal cycling. However, the OVX sheep are in a constant period of anestrus due to the removal of their ovaries and the different treatments will affect the cortical bone remodeling.

Seasonality had a significant effect on the data presented in this study. Analysis of the OVX data showed seasonality in the BV/TV and percent of remodeled tissue. The most porous bone was seen in the winter months, possibly indicative of the inactivity of sheep during the winter months. The inactivity results in lower amounts of loading on the bone which potentially decreases the initiation of the bone remodeling cycle. Spring had a minor recovery period where the porosity decreased. It was not until summer that a full recovery occurred and the BV/TV levels reached a maximum. The higher percentages of remodeled tissue were observed in summer and autumn. This can be attributed to the recovery period where bone remodeling is occurring more frequently to circumvent the more porous bone seen in winter. In addition to seasonal variation, the data for the percent of remodeled tissue had significant anatomical variation. Higher levels of remodeling were seen on the cranial and caudal regions of bone, regions of increased compressive and tensile stress.

Significant seasonality, anatomical variation, and treatment effects were seen during the comparison of the control and OVX sheep. The cement line interface 
measurements for both the tissue and the bone showed significant effects of variation due to season, anatomical region, and treatment. Comparisons of the autumn caudal region and the autumn cranial region indicated no difference in the OVX and the control sheep. However, the autumn caudal and cranial sectors for the OVX sheep were significantly different than the autumn caudal and cranial sectors for the control sheep. This can be explained by the estrous cycle of the ewe that as previously stated begins in November. During the autumn months, the control sheep are able to produce estrogen as opposed to the OVX sheep which cannot. As a result, the OVX bone is much more porous due to the lack of estrogen. On the other hand, no differences were seen in the summer caudal region and the summer cranial region across the control and OVX sheep, indicating treatment did not have an effect. The summer sheep should be approximately four months into their anestrus cycle, at which point estrogen production ceases. This presents an animal that is similar to the OVX sheep and thus supports the study results indicating no differences between the summer months. In addition to supporting the belief of an estrous cycle during autumn and winter, the results also supported Dr. Ruehlman's claims of an estrous cycle during summer and autumn. Looking again at both cement line measurement the differences during the autumn seasons are indicative of the control sheep undergoing estrogen production during those seasons compared to the OVX sheep which would not. However, no differences were seen in any parameter during the summer months.

One limitation of this study is the purchase cohort effect. Upon arrival to Colorado State University, the sheep were already skeletally mature and as a result the previous remodeling cycles must be taken into consideration. To minimize the cohort 
effect, the sheep underwent a post-operative time of 12 months. This was done to allow for the previous remodeling cycles to be remodeled away. Additionally, to maintain uniformity the sheep were all housed in similar conditions using the same feed and exercise conditions.

Another constraint of this study was the projection of a 3D structure onto a 2D plane as seen in the microradiographs. The thickness of the specimen can affect the dimensions of the features being measured. This can present a problem when analyzing the specimen for the histomorphometric measurement as certain features may not be visible within the $2 \mathrm{D}$ plane.

Microradiograph exposure had an effect on the ability to make the histomorphometric measurements. Some sectors were removed because the features of the specimen were not visible. Problems with the exposure also played a role during the densitometry measurement. Issues with sectors being either washed out or too dark were resolved by changing the lighting to optimize visualization and imaging a new step wedge key for that sector. However, even after changing the lighting the key for some sectors did not exhibit a sigmoidal curve and were therefore omitted.

Based on the results from this study, there are potentially many future studies to be considered. One area of interest involves comparison of densitometry data between the control and OVX. This allows for a more complete analysis to understand the effect of ovariectomy on bone density. Another consideration is the use of the OVX sheep for analysis of the bone remodeling cycle in response to estrogen therapy or another therapeutic agent. When compared to the control sheep the hope is that sheep that 
underwent osteoporotic therapy have a similar BMD, thus indicating the effectiveness of the treatment.

Within the OVX data alone, there was significant seasonal and anatomical variation, as well as seasonality within anatomical region. Then when compared to the control sheep there was significant seasonal, anatomical, and treatment differences. In addition, the comparison saw variation due to seasonality within anatomical region, seasonality within treatment, and then an interaction between season, sector and treatment. These results indicate the need for a robust model for compact bone remodeling. The effects of seasonality and region must be considered in the analysis of bone remodeling parameters. As indicated by the results, it cannot be assumed that each region of bone remodels in a similar manner. For example, during comparison of a caudal region and cranial medial region, it would be incorrect to assume that the remodeling in both regions is equivalent. The same can be said for different time points of sheep sacrifice and treatment; the summer OVX and the autumn control sheep did not have comparable amounts of remodeling. As a result, when developing protocols for studies involving bone remodeling and osteoporosis it is important to consider the changes that occur in remodeling due to season, treatment and anatomical region. This research showed that there are varying levels of remodeling in the adult OVX ovine model, provided a model for post-menopausal osteoporosis using 12 month OVX sheep, and indicates that ovariectomy does indeed have an effect on bone remodeling in the ovine model. 


\section{CONCLUSION}

The ovariectomized adult ewe provides an economical large animal model for the study of postmenopausal osteoporosis and compact bone remodeling. Sheep are advantageous because their large size allows for device implantation and studying treatment options for osteoporosis. In addition, sheep have been shown to exhibit Haversian remodeling in the cortical bone 3 to 4 months after the development of plexiform bone. The ovareiectomized model involves removal of the sheep's ovaries simulating menopause, making it an ideal model for studies of postmenopausal osteoporosis.

This research involved the use of ovariectomized sheep divided into four seasonal groups based on time of animal sacrifice, performed at Colorado State University. The animal's right and left radius and ulna were removed and then sent to Henry Ford Hospital. There, the radius was divided into six anatomical sectors and then ground down to a final thickness of $100 \mu \mathrm{m}$ for microradiograph imaging. Finally, an aluminum step wedge was created to provide a key for densitometry analysis. After completion, the microradiographs were sent to California Polytechnic State University, San Luis Obispo for histomorphometric and densitometry analysis.

Histomorphometry involved observation of the microradiographs under white light using a Merz grid to quantify the bone remodeling parameters. The cortical beams for each anatomical sector were divided into four quadrants; measurements were taken in each quadrant and then the values averaged. To quantify remodeling the following measurements were taken: porosity, number of secondary osteons, number of cement line interfaces, and amount of remodeled bone. These measurements were then input into a 
Microsoft Excel spreadsheet to compute the amount of remodeling that occurred in the bone by finding the ratio of bone volume to tissue volume (BV/TV), percent of remodeled tissue and bone, cement line interface of tissue and bone (CLI), and mean secondary osteonal radius.

Densitometry required taking images of each specimens and its corresponding step wedge key. This light intensity and exposure times remained the same for pictures of the aluminum step wedge and the sample. Image analysis for all the pictures was performed to measure the number of pixels in the image at every pixel intensity from 0 to 255. From there mean pixel intensity was found for each level in the step wedge and a key could be determined from this calibration. Mean pixel intensities were also found for each specimen. Then based on the calibration curve and the porosity count, an equivalent thickness of Aluminum (ETA) could be determined for the specimen.

After data collection, statistical analysis was then performed. To analyze the histomorphometric and densitometry measurements for the OVX data, the data was tested using a 2-way Repeated-Measures ANOVA. The ANOVA model was performed to compare the effects of season of sacrifice and anatomical sector on the remodeling parameters. The final statistical test was a 3-way AOVA comparing the OVX sheep with the controls. This model compared the effects of season, treatment, anatomical location, and interactions between all three.

The presented data showed significant seasonal and anatomical variations in compact bone remodeling. The OVX model alone showed seasonal variation in the ratio of bone volume to tissue volume and the percent of remodeled tissue. Anatomical variation was only seen in the percent of remodeled tissue. No demonstrable differences 
due to season or sector were seen in the mean secondary osteon radius. Significant seasonality within anatomical location was exhibited in the percent of remodeled material, and in both cement line interface measurements. Comparison of the OVX and control sheep also resulted in significant seasonal, anatomical, and treatment variations. A significant interaction of season, sector, and treatment were seen in BV/TV, both percent remodeling measurement, and both cement line interface measurements. Seasonal variation was seen in the mean secondary osteon radius. Pairwise comparisons showed similarities and differences between the control and OVX sheep that were indicative of the hormonal changes that occur in the control sheep. The effects of estrogen were seen in the control sheep during their estrous cycles, period of greatest estrogen influence, and comparison of these sheep to the OVX presented significant results.

The large amount of significance indicates there is much variation in the cortical bone remodeling of sheep. It is important that the conclusions of this study be taken into consideration when developing new test protocols for research incorporating the sheep model for studying osteoporosis. The follow up times, treatments, and anatomical region of interest must all be considered because each will have a different effect on the remodeling of the animal. The treatment in relationship to the cyclic hormonal patterns of the sheep also must be clearly understood for better result interpretation. The results of this study showed that varying levels of remodeling occurs in the adult OVX ovine model and differences between the control and OVX model can be attributed to seasonal and anatomical variations, with the polyestrous cycle of the sheep also playing a key role. 


\section{REFERENCES}

1. NOF. National Osteoporsis Foundation: Fast Facts. Available from:

http://www.nof.org/node/40.

2. WHO, WHO Scientific Group on the Assessment of OSteoporosis at Primary Health Care Level. 2004: Brussels.

3. Ammann, P. and R. Rizzoli, Bone strength and its determinants. Osteoporos Int, 2003. 14 Suppl 3: p. S13-8.

4. $\quad$ Poole, K.E.S. and J.E. Compston, Osteoporosis and its management. BMJ, 2006. 333(7581): p. 1251-1256.

5. Marieb, E.N. and K. Hoehn, Human anatomy \& physiology. 2007: Pearson Benjamin Cummings.

6. Bilezikian, J.P., L.G. Raisz, and T.J. Martin, Principles of bone biology. 2008: Elsevier.

7. Martin, R.B., D.B. Burr, and N.A. Sharkey, Skeletal tissue mechanics. 1998: Springer.

8. Nordin, M. and V.H. Frankel, Basic biomechanics of the musculoskeletal system. 2001: Lippincott Williams \& Wilkins.

9. Young, M.F., Bone matrix proteins: their function, regulation, and relationship to osteoporosis. Osteoporos Int, 2003. 14 Suppl 3: p. S35-42.

10. Buchman, S.R., et al., Use of microcomputed tomography scanning as a new technique for the evaluation of membranous bone. The Journal of craniofacial surgery, 1998. 9(1): p. 48-54.

11. Seeman, E. and P.D. Delmas, Bone quality--the material and structural basis of bone strength and fragility. N Engl J Med, 2006. 354(21): p. 2250-61.

12. Eriksen, E.F., et al., Reconstruction of the formative site in iliac trabecular bone in 20 normal individuals employing a kinetic model for matrix and mineral apposition. Metab Bone Dis Relat Res, 1984. 5(5): p. 243-52.

13. Katzenberg, M.A. and S.R. Saunders, Biological anthropology of the human skeleton. 2008: Wiley-Liss.

14. Martin, R.B., Toward a unifying theory of bone remodeling. Bone, 2000. 26(1): p. 1-6.

15. Wolff, J., The Law of Bone Remodelin. Springer, 1986.

16. Beaupre, G.S., T.E. Orr, and D.R. Carter, An approach for time-dependent bone modeling and remodeling--theoretical development. J Orthop Res, 1990. 8(5): p. 651-61.

17. Hedgecock, N.L., et al., Quantitative regional associations between remodeling, modeling, and osteocyte apoptosis and density in rabbit tibial midshafts. Bone, 2007. 40(3): p. 627-637.

18. Abbott Iii, T.A., B.J. Lawrence, and S. Wallach, Osteoporosis: the need for comprehensive treatment guidelines. Clinical Therapeutics, 1996. 18(1): p. 127-149.

19. Fazzalari, N.L., Bone remodeling: A review of the bone microenvironment perspective for fragility fracture (osteoporosis) of the hip. Semin Cell Dev Biol, 2008. 19(5): p. 467-72.

20. Demir, B., et al., Identification of the risk factors for osteoporosis among postmenopausal women. Maturitas, 2008. 60(3-4): p. 253-256.

21. Nelson, H.D., et al., Osteoporosis and Fractures in Postmenopausal Women Using Estrogen. Arch Intern Med, 2002. 162(20): p. 2278-2284.

22. R.L, J., Cytokines, bone remodeling, and estrogen deficiency: a 1998 update. Bone, 1998. 23(2): p. 75-81.

23. Delmas, P.D., Treatment of postmenopausal osteoporosis. The Lancet, 2002. 359(9322): p. 2018-2026.

24. Ego, S., Osteoporosis: Trials and tribulations. The American Journal of Medicine, 1997. 103(2, Supplement 1): p. S74-S89. 
25. Manolagas, S.C., Birth and death of bone cells: basic regulatory mechanisms and implications for the pathogenesis and treatment of osteoporosis. Endocr Rev, 2000. 21(2): p. 115-37.

26. Turner, A.S., Seasonal changes in bone metabolism in sheep: further characterization of an animal model for human osteoporosis. Vet J, 2007. 174(3): p. 460-1.

27. Turner, A.S., Animal models of osteoporosis--necessity and limitations. Eur Cell Mater, 2001. 1: p. 66-81.

28. Feher, A., et al., Bisphosphonates do not inhibit periosteal bone formation in estrogen deficient animals and allow enhanced bone modeling in response to mechanical loading. Bone, 2010. 46(1): p. 203-207.

29. Newman, E., A.S. Turner, and J.D. Wark, The potential of sheep for the study of osteopenia: current status and comparison with other animal models. Bone, 1995. 16(4 Suppl): p. 277S-284S.

30. Baofeng, L., et al., Characterization of a rabbit osteoporosis model induced by ovariectomy and glucocorticoid. Acta Orthopaedica. 81(3): p. 396-401.

31. Arens, D., et al., Seasonal changes in bone metabolism in sheep. Vet J, 2007. 174(3): p. 585-91.

32. Newton, B.I., et al., The Ovariectomized Sheep as a Model for Human Bone Loss. Elsevier, 2004. 130: p. 323-326.

33. Turner, A.S., The sheep as a model for osteoporosis in humans. Vet J, 2002. 163(3): p. 232-9.

34. Turner, A.S., et al., Changes in bone mineral density and bone-specific alkaline phosphatase in ovariectomized ewes. Bone, 1995. 17(4 Suppl): p. 395S-402S.

35. Wu, Z.-x., et al., Effect of ovariectomy on BMD, micro-architecture and biomechanics of cortical and cancellous bones in a sheep model. Medical Engineering \&amp; Physics, 2008. 30(9): p. 1112-1118.

36. Lill, C.A., A.K. Fluegel, and E. Schneider, Effect of ovariectomy, malnutrition and glucocorticoid application on bone properties in sheep: a pilot study. Osteoporos Int, 2002. 13(6): p. 480-6.

37. Calcagno, J., Seasonal and Anatomical Variation in Compact Bone Remodeling in the Adult Bone, in Biomedical Engineering. 2011, California Polytechnic State Univeristy: San Luis Obispo. p. 134.

38. Boivin, G. and P.J. Meunier, The degree of mineralization of bone tissue measured by computerized quantitative contact microradiography. Calcif Tissue Int, 2002. 70(6): p. 503-11.

39. Wessel, N., et al., Short-and Medium-term Estrogen-depletion related Changes in Anatomic Variation of Time-dependent Material Properties, and Evidence of Compact Bone Secondary remodeling. Orthopedic Research Society, 2010: p. 580.

40. Yates, A.J., et al., Radiographic absorptiometry in the diagnosis of osteoporosis. The American Journal of Medicine, 1995. 98(2, Supplement 1): p. 41S-47S.

41. Wessel, N., et al., Short- and Medium-term Estrogen-depletion-related Changes in Anatomic Variation of Time-dependent Materials Properties, and Evidence of Compact Bone Secondary Remodeling. Orthopedic Research Society, 2010: p. 580.

42. Lanyon, L.E., P.T. Magee, and D.G. Baggott, The relationship of functional stress and strain to the processes of bone remodelling. An experimental study on the sheep radius. Journal of Biomechanics, 1979. 12(8): p. 593-600.

43. Parsons, L.C., Osteoporosis: Incidence, Prevention, and Treatment of the Silent Killer. Nursing Clinics of North America, 2005. 40(1): p. 119-133.

44. Ruan, W.-d., et al., Analysis on the risk factors of second fracture in osteoporosis-related fractures. Chinese Journal of Traumatology (English Edition), 2011. 14(2): p. 74-78. 
45. Augat, P., et al., Osteoporosis prevalence and fracture characteristics in elderly female patients with fractures. Archives of Orthopaedic and Trauma Surgery, 2010. 130(11): p. 1405-1410.

46. Lespessailles, E., et al., Imaging techniques for evaluating bone microarchitecture. Joint Bone Spine, 2006. 73(3): p. 254-61.

47. Mosekilde, L., Assessing bone quality - Animal models in preclinical osteoporosis research. Bone, 1995. 17(4, Supplement): p. S343-S352.

48. Boutroy, S., et al., In Vivo Assessment of Trabecular Bone Microarchitecture by HighResolution Peripheral Quantitative Computed Tomography. Journal of Clinical Endocrinology \& Metabolism, 2005. 90(12): p. 6508-6515.

49. Thorndike, E.A. and A.S. Turner, In search of an animal model for postmenopausal diseases. Front Biosci, 1998. 3: p. c17-26.

50. Arendt, J., A. Symons, and C. Laud, Pineal function in the sheep: evidence for a possible mechanism mediating seasonal reproductive activity. Cellular and Molecular Life Sciences, 1981. 37(6): p. 584-586.

51. Robinson, J.E., H.M. Radford, and F.J. Karsch, Seasonal changes in pulsatile luteinizing hormone (LH) secretion in the ewe: relationship of frequency of LH pulses to day length and response to estradiol negative feedback. Biology of Reproduction, 1985. 33(2): p. 324-334.

52. Ruehlman, D.L., Reproductive Cylce of Sheep, C.M. Les, Editor. 2012. 
Appendix A: 12 Month OVX Histomorphometry Data

\begin{tabular}{|c|c|c|c|c|c|c|}
\hline \multicolumn{7}{|c|}{12 Month Autumn OVX } \\
\hline \multicolumn{7}{|c|}{ Average BV/TV } \\
\hline \multicolumn{7}{|c|}{ Sector } \\
\hline Sheep & Craniomedial & Cranial & Craniolateral & Caudomedial & Caudal & Caudolateral \\
\hline $\mathrm{C} 29$ & 0.875 & 0.8958 & 0.9236 & 0.8889 & 0.9236 & 0.9514 \\
\hline $\mathrm{C} 30$ & 0.9236 & 0.9306 & 0.9097 & 0.9167 & 0.9514 & 0.9583 \\
\hline $\mathrm{C} 33$ & 0.8889 & 0.9444 & 0.9306 & 0.9167 & 0.9444 & 0.9444 \\
\hline C39 & 0.9306 & 0.9167 & 0.9236 & 0.875 & 0.9444 & 0.9167 \\
\hline $\mathrm{C} 40$ & 0.9306 & 0.9352 & 0.9236 & 0.9375 & 0.9236 & 0.9306 \\
\hline $\mathrm{C} 42$ & 0.9444 & 0.9653 & 0.9306 & 0.9236 & 0.9583 & 0.9583 \\
\hline \multicolumn{7}{|c|}{ Average Percent Remodeled (Tissue) (Remodeled Tissue/Total Area) } \\
\hline & \multicolumn{6}{|c|}{ Sector } \\
\hline Sheep & Craniomedial & Cranial & Craniolateral & Caudomedial & Caudal & Caudolatera \\
\hline $\mathrm{C} 29$ & 0.014 & 0.104 & \begin{tabular}{|l|}
0.222 \\
\end{tabular} & 0.208 & 0.424 & 0.188 \\
\hline $\mathrm{C} 30$ & 0.431 & 0.479 & 0.194 & 0.514 & 0.417 & 0.333 \\
\hline $\mathrm{C} 33$ & 0.257 & 0.396 & 0.451 & 0.354 & 0.313 & 0.333 \\
\hline $\mathrm{C} 39$ & 0.472 & 0.285 & 0.361 & 0.521 & 0.396 & 0.549 \\
\hline $\mathrm{C} 40$ & 0.299 & 0.324 & 0.403 & 0.340 & 0.375 & 0.319 \\
\hline $\mathrm{C} 42$ & 0.458 & 0.201 & 0.236 & 0.313 & 0.389 & 0.264 \\
\hline \multicolumn{7}{|c|}{ Average Percent Remodeled (Material) (Remodeled Tissue/Bone Area) } \\
\hline & \multicolumn{6}{|c|}{ Sector } \\
\hline Sheep & Craniomedial & Cranial & Craniolateral & Caudomedial & Caudal & Caudolatera \\
\hline $\mathrm{C} 29$ & 0.147 & 0.116 & 0.241 & 0.239 & 0.458 & 0.197 \\
\hline $\mathrm{C} 30$ & 0.465 & 0.516 & 0.212 & 0.565 & 0.438 & 0.350 \\
\hline $\mathrm{C} 33$ & 0.290 & 0.417 & 0.486 & 0.385 & 0.331 & 0.354 \\
\hline $\mathrm{C} 39$ & 0.506 & 0.313 & 0.292 & 0.594 & 0.419 & 0.601 \\
\hline $\mathrm{C} 40$ & 0.321 & 0.347 & 0.437 & 0.365 & 0.406 & 0.343 \\
\hline $\mathrm{C} 42$ & 0.490 & 0.208 & 0.254 & 0.339 & 0.408 & 0.276 \\
\hline \multicolumn{7}{|c|}{ Average Osteon Radius $(\mu \mathrm{m})$} \\
\hline & \multicolumn{6}{|c|}{ Sector } \\
\hline Sheep & Craniomedial & Cranial & Craniolateral & Caudomedial & Caudal & Caudolatera \\
\hline $\mathrm{C} 29$ & 18.689 & 51.235 & 55.840 & 60.763 & 67.174 & 59.269 \\
\hline $\mathrm{C} 30$ & 58.741 & 63.080 & 49.277 & 56.816 & 56.576 & 64.230 \\
\hline $\mathrm{C} 33$ & 50.852 & 63.108 & 60.450 & 53.229 & 68.235 & 63.517 \\
\hline $\mathrm{C} 39$ & 83.165 & 56.682 & 66.343 & 78.244 & 73.216 & 72.045 \\
\hline $\mathrm{C} 40$ & 47.703 & 56.160 & 65.152 & 52.142 & 60.981 & 56.311 \\
\hline
\end{tabular}




\begin{tabular}{|c|c|c|c|c|c|c|}
\hline \multicolumn{7}{|c|}{ Average CLI (Tissue) $\left(\mathbf{m m} / \mathbf{m m}^{2}\right)$} \\
\hline \multicolumn{7}{|c|}{ Sector } \\
\hline Sheep & Craniomedial & Cranial & Craniolateral & Caudomedial & Caudal & Caudolateral \\
\hline C29 & 0.750 & 11.250 & 26.750 & 22.250 & 33.000 & 15.750 \\
\hline C30 & 14.000 & 19.500 & 23.250 & 19.000 & 23.500 & 17.250 \\
\hline C33 & 30.750 & 43.750 & 47.750 & 41.250 & 27.250 & 35.500 \\
\hline C39 & 23.250 & 26.250 & 26.250 & 31.250 & 31.000 & 27.750 \\
\hline C40 & 8.250 & 13.000 & 21.000 & 19.500 & 30.250 & 30.750 \\
\hline C42 & 25.000 & 18.500 & 19.750 & 12.750 & 29.500 & 11.250 \\
\hline \multicolumn{7}{|c|}{ Average CLI (Material) (mm/mm $\left.{ }^{2}\right)$} \\
\hline \multicolumn{7}{|c|}{ Sector } \\
\hline Sheep & Craniomedial & Cranial & Craniolateral & Caudomedial & Caudal & Caudolateral \\
\hline C29 & 0.865 & 12.453 & 29.027 & 25.458 & 35.868 & 16.571 \\
\hline C30 & 15.098 & 20.912 & 25.353 & 20.890 & 24.724 & 17.912 \\
\hline C33 & 34.728 & 46.100 & 51.313 & 44.778 & 29.014 & 37.544 \\
\hline C39 & 24.866 & 28.817 & 28.382 & 36.050 & 32.878 & 30.053 \\
\hline C40 & 8.847 & 14.909 & 22.734 & 20.790 & 32.679 & 33.142 \\
\hline C42 & 26.507 & 19.141 & 21.172 & 13.754 & 30.736 & 11.736 \\
\hline
\end{tabular}

\begin{tabular}{|c|c|c|c|c|c|c|}
\hline \multicolumn{7}{|c|}{ 12 Month Winter OVX } \\
\hline \hline \multicolumn{7}{|c|}{ Average BV/TV } \\
\hline & Cranial & Craniolateral & Caudomedial & Caudal & Caudolateral \\
\hline Sheep & Craniomedial & Cector \\
\hline C58 & 0.9097 & 0.9375 & 0.9167 & 0.9236 & 0.8472 & 0.9444 \\
\hline C62 & 0.8819 & 0.9236 & 0.9514 & 0.9028 & 0.9306 & 0.8958 \\
\hline C67 & 0.9306 & 0.9167 & 0.9792 & 0.9236 & 0.9306 & 0.9236 \\
\hline C68 & 0.8889 & 0.9306 & 0.9306 & 0.9653 & 0.9375 & 0.9236 \\
\hline C70 & 0.9306 & 0.9514 & 0.9375 & 0.9583 & 0.875 & 0.9236 \\
\hline C71 & 0.9236 & 0.8542 & 0.8542 & 0.8681 & 0.9236 & 0.9514 \\
\hline \multicolumn{7}{|c|}{ Average Percent Remodeled (Tissue) (Remodeled Tissue/Total Area) } \\
\hline Sheep & Craniomedial & Cranial & Craniolateral & Caudomedial & Caudal & Caudolateral \\
\hline C58 & 0.194 & 0.139 & 0.174 & 0.396 & 0.174 & 0.333 \\
\hline C62 & 0.097 & 0.229 & 0.208 & 0.229 & 0.257 & 0.111 \\
\hline C67 & 0.194 & 0.188 & 0.208 & 0.306 & 0.306 & 0.104 \\
\hline C68 & 0.201 & 0.354 & 0.188 & 0.271 & 0.424 & 0.174 \\
\hline C70 & 0.292 & 0.438 & 0.410 & 0.347 & 0.319 & 0.243 \\
\hline
\end{tabular}




\begin{tabular}{|c|c|c|c|c|c|c|}
\hline C71 & 0.201 & 0.285 & 0.375 & 0.438 & 0.493 & 0.458 \\
\hline \multicolumn{7}{|c|}{ Average Percent Remodeled (Material) (Remodeled Tissue/Bone Area) } \\
\hline & \multicolumn{6}{|c|}{ Sector } \\
\hline Sheep & Craniomedial & Cranial & Craniolateral & Caudomedial & Caudal & Caudolateral \\
\hline C58 & 0.215 & 0.150 & 0.189 & 0.430 & 0.212 & 0.361 \\
\hline C62 & 0.108 & 0.251 & 0.218 & 0.256 & 0.273 & 0.123 \\
\hline C67 & 0.207 & 0.201 & 0.214 & 0.334 & 0.328 & 0.113 \\
\hline C68 & 0.234 & 0.380 & 0.205 & 0.280 & 0.457 & 0.193 \\
\hline $\mathrm{C} 70$ & 0.315 & 0.461 & 0.437 & 0.364 & 0.365 & 0.264 \\
\hline C71 & 0.216 & 0.344 & 0.442 & 0.507 & 0.534 & 0.481 \\
\hline \multicolumn{7}{|c|}{ Average Osteon Radius $(\mu \mathrm{m})$} \\
\hline & \multicolumn{6}{|c|}{ Sector } \\
\hline Sheep & Craniomedial & Cranial & Craniolateral & Caudomedial & Caudal & Caudolateral \\
\hline $\mathrm{C} 58$ & 57.386 & 43.298 & 56.208 & 74.487 & 64.944 & 65.268 \\
\hline C62 & 54.969 & 45.995 & 52.048 & 51.418 & 50.025 & 47.594 \\
\hline C67 & 47.907 & 47.506 & 52.994 & 53.833 & 61.040 & 58.271 \\
\hline C68 & 49.772 & 71.154 & 49.411 & 55.087 & 67.030 & 46.696 \\
\hline $\mathrm{C} 70$ & 53.920 & 62.140 & 67.358 & 56.877 & 68.641 & 54.287 \\
\hline C71 & 43.897 & 65.277 & 62.983 & 65.213 & 66.991 & 70.713 \\
\hline \multicolumn{7}{|c|}{ Average CLI (Tissue) $\left(\mathrm{mm} / \mathrm{mm}^{2}\right)$} \\
\hline & \multicolumn{6}{|c|}{ Sector } \\
\hline Sheep & Craniomedial & Cranial & Craniolateral & Caudomedial & Caudal & Caudolateral \\
\hline $\mathrm{C} 58$ & 15.000 & 20.000 & 15.000 & 27.000 & 16.500 & 25.000 \\
\hline C62 & 7.000 & 19.500 & 19.250 & 25.750 & 23.500 & 11.250 \\
\hline C67 & 21.000 & 21.500 & 21.500 & 25.750 & 25.000 & 8.750 \\
\hline C68 & 21.500 & 24.250 & 21.250 & 21.250 & 32.500 & 18.000 \\
\hline $\mathrm{C} 70$ & 36.000 & 37.750 & 28.250 & 35.000 & 28.000 & 24.250 \\
\hline C71 & 23.750 & 27.250 & 28.000 & 37.000 & 36.000 & 29.250 \\
\hline \multicolumn{7}{|c|}{ Average CLI (Material) $\left(\mathrm{mm} / \mathrm{mm}^{2}\right)$} \\
\hline & \multicolumn{6}{|c|}{ Sector } \\
\hline Sheep & Craniomedial & Cranial & Craniolateral & Caudomedial & Caudal & Caudolateral \\
\hline $\mathrm{C} 58$ & 16.457 & 21.299 & 16.261 & 29.007 & 19.926 & 27.143 \\
\hline C62 & 7.955 & 21.187 & 20.238 & 28.737 & 25.329 & 12.446 \\
\hline C67 & 22.467 & 23.140 & 21.900 & 27.596 & 26.727 & 9.406 \\
\hline C68 & 24.835 & 25.922 & 23.227 & 21.987 & 34.889 & 19.700 \\
\hline $\mathrm{C} 70$ & 38.621 & 39.457 & 30.157 & 36.616 & 31.372 & 26.254 \\
\hline C71 & 25.438 & 33.748 & 32.643 & 42.332 & 39.122 & 30.812 \\
\hline
\end{tabular}


12 Month Spring OVX

Average BV/TV

\begin{tabular}{|c|c|c|c|c|c|c|}
\hline & \multicolumn{6}{|c|}{ Sector } \\
\hline Sheep & Craniomedial & Cranial & Craniolateral & Caudomedial & Caudal & Caudolateral \\
\hline C101 & 0.9167 & 0.9792 & 0.9167 & 0.9444 & 0.8958 & 0.9514 \\
\hline C102 & 0.9236 & 0.9306 & 0.9514 & 0.875 & 0.9375 & 0.9028 \\
\hline C104 & 0.9653 & 0.9167 & 0.9375 & 0.9306 & 0.9236 & 0.875 \\
\hline C111 & 0.9583 & 0.9444 & 0.9028 & 0.8958 & 0.8681 & 0.9375 \\
\hline C112 & 0.9236 & 0.9352 & 0.8958 & 0.8958 & 0.9236 & 0.9444 \\
\hline C89 & 0.9861 & 0.9444 & 0.9306 & 0.9306 & 0.9653 & 0.9167 \\
\hline C98 & 0.9444 & 0.9514 & 0.9514 & 0.9514 & 0.9444 & 0.9514 \\
\hline
\end{tabular}

Average Percent Remodeled (Tissue) (Remodeled Tissue/Total Area)

\begin{tabular}{|c|c|c|c|c|c|c|}
\hline & \multicolumn{6}{|c|}{ Sector } \\
\hline Sheep & Craniomedial & Cranial & Craniolateral & Caudomedial & Caudal & Caudolateral \\
\hline C101 & 0.243 & 0.201 & 0.285 & 0.174 & 0.243 & 0.382 \\
\hline C102 & 0.076 & 0.194 & 0.083 & 0.021 & 0.389 & 0.069 \\
\hline $\mathrm{C} 104$ & 0.201 & 0.264 & 0.049 & 0.125 & 0.229 & 0.139 \\
\hline C111 & 0.146 & 0.292 & 0.340 & 0.118 & 0.278 & 0.389 \\
\hline C112 & 0.181 & 0.361 & 0.229 & 0.264 & 0.208 & 0.069 \\
\hline C89 & 0.326 & 0.104 & 0.035 & 0.076 & 0.236 & 0.264 \\
\hline C98 & 0.306 & 0.174 & 0.056 & 0.285 & 0.313 & 0.292 \\
\hline \multicolumn{7}{|c|}{ Average Percent Remodeled (Material) (Remodeled Tissue/Bone Area) } \\
\hline & \multicolumn{6}{|c|}{ Sector } \\
\hline Sheep & Craniomedial & Cranial & Craniolateral & Caudomedial & Caudal & Caudolateral \\
\hline $\mathrm{C} 101$ & 0.275 & 0.207 & 0.308 & 0.183 & 0.273 & 0.400 \\
\hline C102 & 0.086 & 0.207 & 0.087 & 0.025 & 0.415 & 0.080 \\
\hline C104 & 0.209 & 0.289 & 0.053 & 0.145 & 0.251 & 0.161 \\
\hline $\mathrm{C} 111$ & 0.160 & 0.308 & 0.379 & 0.133 & 0.323 & 0.413 \\
\hline C112 & 0.197 & 0.388 & 0.258 & 0.298 & 0.224 & 0.075 \\
\hline C89 & 0.329 & 0.111 & 0.037 & 0.081 & 0.245 & 0.292 \\
\hline C98 & 0.323 & 0.187 & 0.059 & 0.299 & 0.331 & 0.307 \\
\hline \multicolumn{7}{|c|}{ Average Osteon Radius $(\mu \mathrm{m})$} \\
\hline & \multicolumn{6}{|c|}{ Sector } \\
\hline Sheep & Craniomedial & Cranial & Craniolateral & Caudomedial & Caudal & Caudolateral \\
\hline C101 & 0.000 & 0.000 & 61.334 & 61.553 & 57.048 & 62.791 \\
\hline $\mathrm{C} 102$ & 43.404 & 54.632 & 39.942 & 26.319 & 62.853 & 47.628 \\
\hline C104 & 53.981 & 60.066 & 43.153 & 0.000 & 54.259 & 56.263 \\
\hline C111 & 0.000 & 79.082 & 78.083 & 54.093 & 65.259 & 70.099 \\
\hline C112 & 54.675 & 61.875 & 50.512 & 59.552 & 51.382 & 40.742 \\
\hline
\end{tabular}




\begin{tabular}{|c|c|c|c|c|c|c|}
\hline C89 & 62.125 & 44.694 & 31.154 & 35.042 & 61.359 & 47.537 \\
\hline C98 & 68.650 & 55.271 & 0.000 & 70.698 & 65.243 & 59.029 \\
\hline \multicolumn{7}{|c|}{ Average CLI (Tissue) $\left(\mathbf{m m} \mathbf{m m m}^{\mathbf{2}}\right)$} \\
\hline \multicolumn{7}{|c|}{ Sector } \\
\hline Sheep & Craniomedial & Cranial & Craniolateral & Caudomedial & Caudal & Caudolateral \\
\hline C101 & 27.333 & 30.333 & 26.750 & 15.000 & 28.750 & 38.500 \\
\hline C102 & 11.500 & 17.750 & 12.250 & 5.750 & 31.250 & 10.000 \\
\hline C104 & 25.000 & 27.250 & 6.000 & 18.333 & 29.250 & 13.750 \\
\hline C111 & 18.500 & 26.000 & 25.750 & 10.000 & 25.750 & 29.750 \\
\hline C112 & 15.500 & 30.750 & 27.500 & 25.500 & 25.000 & 6.000 \\
\hline C89 & 31.000 & 16.500 & 6.250 & 12.750 & 25.000 & 30.500 \\
\hline C98 & 26.500 & 18.250 & 9.000 & 22.750 & 27.750 & 32.000 \\
\hline \multicolumn{7}{|c|}{ Average CLI (Material) (mm/mm $)$} \\
\hline \multicolumn{7}{|c|}{ Sector } \\
\hline Sheep & Craniomedial & Cranial & Craniolateral & Caudomedial & Caudal & Caudolateral \\
\hline C101 & 23.069 & 23.493 & 29.123 & 15.912 & 32.086 & 40.551 \\
\hline C102 & 12.749 & 19.086 & 12.865 & 6.565 & 33.361 & 11.271 \\
\hline C104 & 26.069 & 29.824 & 6.663 & 16.258 & 31.855 & 15.655 \\
\hline C111 & 10.042 & 27.442 & 28.548 & 11.141 & 29.832 & 31.617 \\
\hline C112 & 16.838 & 30.849 & 30.912 & 27.849 & 22.423 & 14.818 \\
\hline C89 & 28.138 & 12.828 & 11.182 & 13.555 & 31.760 & 28.102 \\
\hline C98 & 24.015 & 17.880 & 13.423 & 23.349 & 32.081 & 33.600 \\
\hline
\end{tabular}

\begin{tabular}{|c|c|c|c|c|c|c|}
\hline \multicolumn{7}{|c|}{ 12 Month Summer OVX } \\
\hline \hline \multicolumn{7}{|c|}{ Average BV/TV } \\
\hline & Cranial & Craniolateral & Caudomedial & Caudal & Caudolateral \\
\hline Sheep & Craniomedial & Cector \\
\hline C04 & 0.917 & 0.965 & 0.951 & 0.965 & 0.868 & 0.965 \\
\hline C05 & 0.965 & 0.958 & 0.986 & 0.958 & 0.958 & 0.969 \\
\hline C08 & 0.944 & 0.965 & 0.972 & 0.944 & 0.972 & 0.979 \\
\hline C13 & 0.972 & 0.938 & 0.958 & 0.979 & 0.935 & 0.824 \\
\hline C18 & 0.979 & 0.979 & 0.951 & 0.979 & 0.944 & 0.972 \\
\hline \multicolumn{7}{|c|}{ Average Percent Remodeled (Tissue) (Remodeled Tissue/Total Area) } \\
\hline Sheep & Craniomedial & Cranial & Craniolateral & Caudomedial & Caudal & Caudolateral \\
\hline C04 & 0.181 & 0.375 & 0.153 & 0.118 & 0.278 & 0.125 \\
\hline C05 & 0.076 & 0.424 & 0.097 & 0.458 & 0.556 & 0.424 \\
\hline C08 & 0.028 & 0.465 & 0.398 & 0.278 & 0.481 & 0.438 \\
\hline C13 & 0.120 & 0.496 & 0.139 & 0.410 & 0.361 & 0.602 \\
\hline
\end{tabular}




\begin{tabular}{|c|c|c|c|c|c|c|}
\hline $\mathrm{C} 18$ & 0.465 & 0.326 & 0.174 & 0.417 & 0.458 & 0.333 \\
\hline \multicolumn{7}{|c|}{ Average Percent Remodeled (Material) (Remodeled Tissue/Bone Area) } \\
\hline & \multicolumn{6}{|c|}{ Sector } \\
\hline Sheep & Craniomedial & Cranial & Craniolateral & Caudomedial & Caudal & Caudolateral \\
\hline $\mathrm{C} 04$ & 0.196 & 0.389 & 0.161 & 0.122 & 0.322 & 0.130 \\
\hline $\mathrm{C} 05$ & 0.080 & 0.444 & 0.099 & 0.478 & 0.579 & 0.432 \\
\hline $\mathrm{C} 08$ & 0.029 & 0.481 & 0.408 & 0.292 & 0.494 & 0.449 \\
\hline $\mathrm{C} 13$ & 0.124 & 0.519 & 0.145 & 0.422 & 0.386 & 0.731 \\
\hline $\mathrm{C} 18$ & 0.474 & 0.333 & 0.183 & 0.425 & 0.485 & 0.342 \\
\hline \multicolumn{7}{|c|}{ Average Osteon Radius $(\mu \mathrm{m})$} \\
\hline & \multicolumn{6}{|c|}{ Sector } \\
\hline Sheep & Craniomedial & Cranial & Craniolateral & Caudomedial & Caudal & Caudolateral \\
\hline $\mathrm{C} 04$ & 94.020 & 52.460 & 67.630 & 41.440 & 39.040 & 29.710 \\
\hline $\mathrm{C} 05$ & 32.120 & 57.670 & 45.210 & 94.540 & 81.020 & 56.270 \\
\hline $\mathrm{C} 08$ & 44.140 & 136.050 & 116.100 & 82.380 & 63.870 & 84.640 \\
\hline $\mathrm{C} 13$ & 47.320 & 60.680 & 52.140 & 63.990 & 60.210 & 71.240 \\
\hline $\mathrm{C} 18$ & 65.320 & 56.340 & 62.490 & 66.610 & 59.150 & 49.840 \\
\hline \multicolumn{7}{|c|}{ Average CLI (Tissue) $\left(\mathrm{mm} / \mathrm{mm}^{2}\right)$} \\
\hline & \multicolumn{6}{|c|}{ Sector } \\
\hline Sheep & Craniomedial & Cranial & Craniolateral & Caudomedial & Caudal & Caudolateral \\
\hline $\mathrm{C} 04$ & 19.000 & 42.000 & 14.250 & 11.250 & 26.500 & 8.750 \\
\hline $\mathrm{C} 05$ & 5.750 & 49.250 & 8.000 & 38.250 & 62.750 & 55.250 \\
\hline $\mathrm{C} 08$ & 4.000 & 51.500 & 43.440 & 22.500 & 47.000 & 45.250 \\
\hline $\mathrm{C} 13$ & 12.330 & 46.500 & 15.000 & 44.250 & 37.000 & 48.000 \\
\hline $\mathrm{C} 18$ & 50.000 & 30.500 & 20.750 & 36.250 & 50.000 & 38.750 \\
\hline \multicolumn{7}{|c|}{ Average CLI (Material) $\left(\mathrm{mm} / \mathrm{mm}^{2}\right)$} \\
\hline & \multicolumn{6}{|c|}{ Sector } \\
\hline Sheep & Craniomedial & Cranial & Craniolateral & Caudomedial & Caudal & Caudolateral \\
\hline $\mathrm{C} 04$ & 20.570 & 43.480 & 15.120 & 11.650 & 30.520 & 9.040 \\
\hline $\mathrm{C} 05$ & 6.000 & 51.760 & 8.150 & 39.820 & 65.490 & 56.400 \\
\hline $\mathrm{C} 08$ & 4.230 & 53.430 & 44.690 & 23.810 & 48.340 & 46.230 \\
\hline $\mathrm{C} 13$ & 12.780 & 49.670 & 15.740 & 45.390 & 39.610 & 58.320 \\
\hline $\mathrm{C} 18$ & 53.570 & 31.160 & 21.760 & 37.050 & 52.970 & 39.860 \\
\hline
\end{tabular}


Appendix B: 12 month OVX Densitometry Data

\begin{tabular}{|c|c|c|c|c|c|c|}
\hline \multicolumn{7}{|c|}{ 12 Month Autumn OVX } \\
\hline \hline \multicolumn{7}{|c|}{ ETA (mm) } \\
\hline Sheep & Craniomedial & Cranial & Craniolateral & Caudomedial & Caudal & Caudolateral \\
\hline C29 & 0.0938 & 0.0862 & 0.6807 & 0.0828 & 0.0720 & 0.0889 \\
\hline C30 & 0.0584 & 0.0543 & 0.8727 & 0.0653 & 0.0598 & 0.4889 \\
\hline C33 & 0.0712 & 0.0681 & 0.0592 & 0.0774 & 0.0637 & 0.0689 \\
\hline C39 & 0.0585 & 0.0570 & 0.0526 & 0.0427 & 0.0490 & 0.0560 \\
\hline C40 & 0.0721 & 0.0728 & 0.0773 & 0.0570 & 0.0765 & 0.0788 \\
\hline C42 & 0.0372 & 0.0637 & 0.0685 & 0.0597 & 0.0550 & 0.0666 \\
\hline
\end{tabular}

\begin{tabular}{|c|c|c|c|c|c|c|}
\hline \multicolumn{7}{|c|}{ 12 Month Winter OVX } \\
\hline \hline \multicolumn{7}{|c|}{ ETA (mm) } \\
\hline Sheep & Craniomedial & Cranial & Craniolateral & Caudomedial & Caudal & Caudolateral \\
\hline C58 & 0.0662 & 0.0727 & 0.0736 & 0.0724 & 0.0000 & 0.0751 \\
\hline C62 & 0.0650 & 0.0649 & 0.0754 & 0.0703 & 0.0646 & 0.0692 \\
\hline C67 & 0.0768 & 0.0644 & 0.0677 & 0.0814 & 0.0709 & 0.0741 \\
\hline C68 & 0.0745 & 0.0663 & 0.0742 & 0.0584 & 0.0564 & 0.0725 \\
\hline C70 & 0.0486 & 0.0324 & 0.0389 & 0.0246 & 0.0315 & 0.0448 \\
\hline C71 & 0.0521 & 0.0436 & 0.0475 & 0.0503 & 0.0430 & 0.0414 \\
\hline
\end{tabular}

\begin{tabular}{|c|c|c|c|c|c|c|}
\hline \multicolumn{7}{|c|}{ 12 Month Spring OVX } \\
\hline \hline \multicolumn{7}{|c|}{ ETA (mm) } \\
\hline Sheep & Craniomedial & Cranial & Craniolateral & Caudomedial & Caudal & Caudolateral \\
\hline C101 & 0.0682 & 0.0639 & 0.0644 & 0.0781 & 0.0577 & 0.0582 \\
\hline C102 & 0.0585 & 0.0600 & 0.0798 & 0.0677 & 0.0663 & 0.0725 \\
\hline C104 & 0.0640 & 0.0665 & 0.0765 & 0.0865 & 0.0679 & 0.0738 \\
\hline C111 & 0.0752 & 0.0504 & 0.0547 & 0.0762 & 0.0623 & 0.0587 \\
\hline C112 & 0.0808 & 0.0750 & 0.0777 & 0.0775 & 0.0776 & 0.0846 \\
\hline C89 & 0.0652 & 0.0499 & 0.0637 & 0.0574 & 0.0712 & 0.0505 \\
\hline C98 & 0.0656 & 0.0407 & 0.0307 & 0.0555 & 0.0415 & 0.0511 \\
\hline
\end{tabular}


12 Month Summer OVX

\begin{tabular}{|c|c|c|c|c|c|c|}
\hline \hline \multicolumn{7}{|c|}{ ETA $(\mathrm{mm})$} \\
\hline & \multicolumn{7}{|c|}{ Sector } \\
\hline Sheep & Craniomedial & Cranial & Craniolateral & Caudomedial & Caudal & Caudolateral \\
\hline C04 & 0.0656 & 0.0431 & 0.0445 & 0.0376 & 0.0461 & 0.0648 \\
\hline C05 & 0.0793 & 0.0633 & 0.0817 & 0.0586 & 0.0613 & 0.0546 \\
\hline C08 & 0.0440 & 0.0407 & 0.0258 & 0.0390 & 0.0266 & 0.0346 \\
\hline C13 & 0.0481 & 0.0646 & 0.0765 & 0.0632 & 0.1213 & 0.0494 \\
\hline C18 & 0.0776 & 0.0710 & 0.0684 & 0.0665 & 0.0608 & 0.0740 \\
\hline
\end{tabular}

